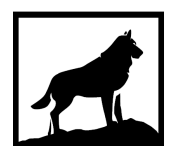

Michigan Technological

1 8 8 5 University
Michigan Technological University Digital Commons @ Michigan Tech

\title{
ANALYTICAL ASSESSMENT OF PROJECT COMPLEXITY: A GUIDE TO MANAGING PROJECTS THROUGH REDUCTION OF COMPLEXITY
}

Christopher VanArsdale

Michigan Technological University, cdvanars@mtu.edu

Copyright 2021 Christopher VanArsdale

\section{Recommended Citation}

VanArsdale, Christopher, "ANALYTICAL ASSESSMENT OF PROJECT COMPLEXITY: A GUIDE TO MANAGING PROJECTS THROUGH REDUCTION OF COMPLEXITY", Open Access Dissertation, Michigan Technological University, 2021.

https://doi.org/10.37099/mtu.dc.etdr/1162

Follow this and additional works at: https://digitalcommons.mtu.edu/etdr

Part of the Construction Engineering and Management Commons, Other Operations Research, Systems Engineering and Industrial Engineering Commons, and the Systems Engineering Commons 
ANALYTICAL ASSESSMENT OF PROJECT COMPLEXITY: A GUIDE TO MANAGING PROJECTS THROUGH REDUCTION OF COMPLEXITY

By

Christopher Van Arsdale

\begin{abstract}
A DISSERTATION
Submitted in partial fulfillment of the requirements for the degree of

DOCTOR OF PHILOSOPHY

In Civil Engineering
\end{abstract}

MICHIGAN TECHNOLOGICAL UNIVERSITY

2021

(C) 2021 Christopher Van Arsdale 
This dissertation has been approved in partial fulfillment of the requirements for the Degree of DOCTOR OF PHILOSOPHY in Civil Engineering.

Department of Civil and Environmental Engineering

\author{
Dissertation Advisor: Dr. Amlan Mukherjee \\ Committee Member: Dr. William Bulleit \\ Committee Member: Dr. Ann Maclean \\ Committee Member: Dr. Raymond A. Swartz \\ Department Chair: Dr. Audra Morse
}




\section{Table of Contents}

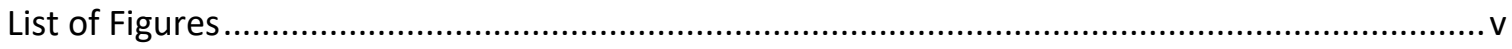

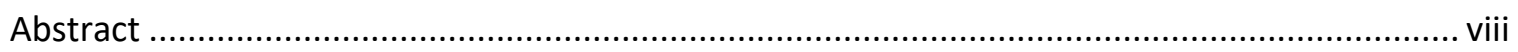

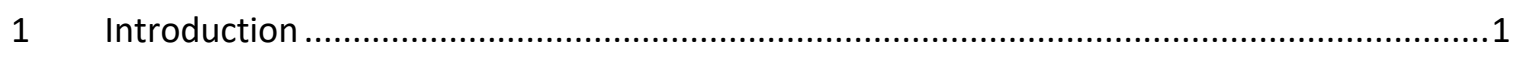

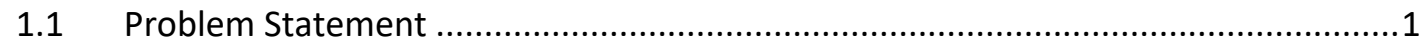

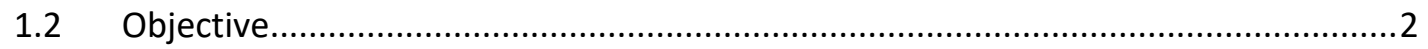

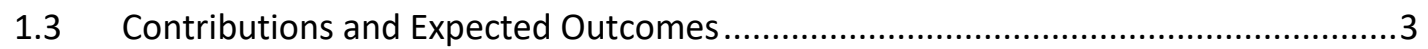

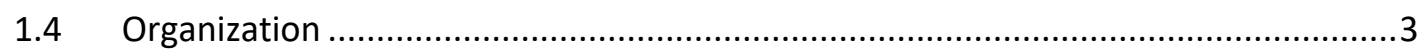

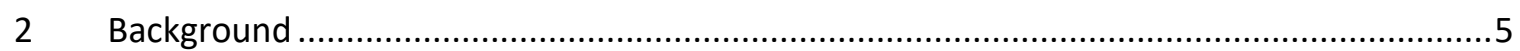

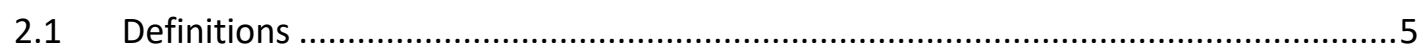

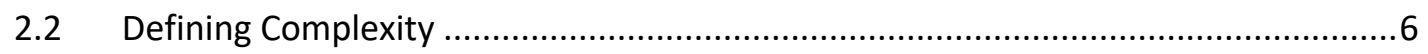

2.3 Foundational Work: Measuring Complexity .......................................................

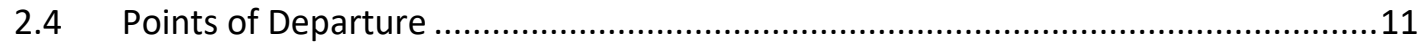

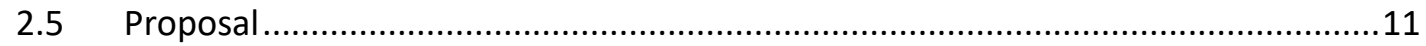

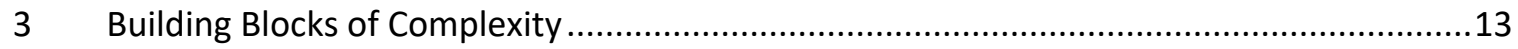

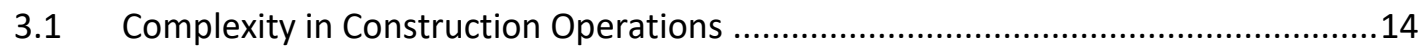

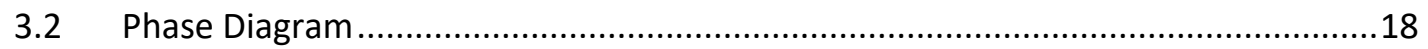

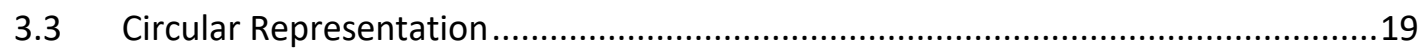

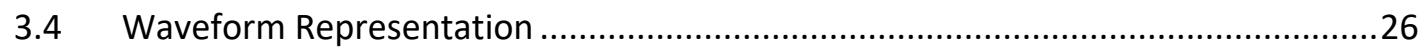

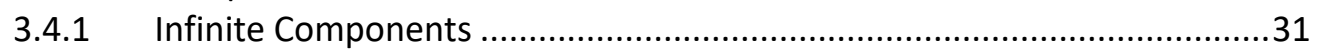

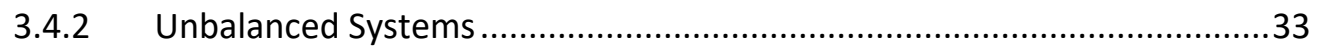

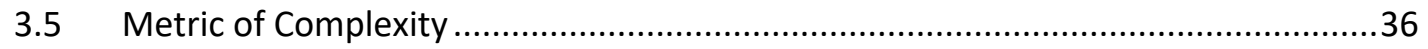

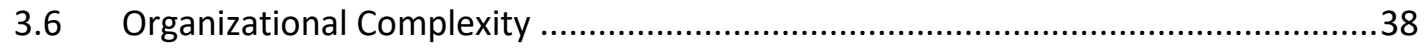

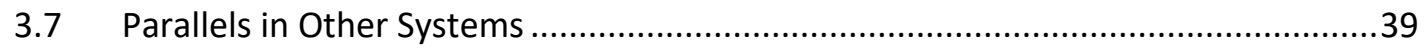

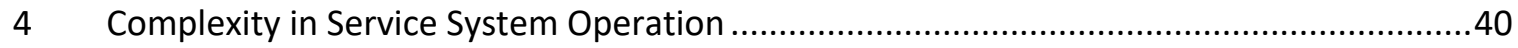

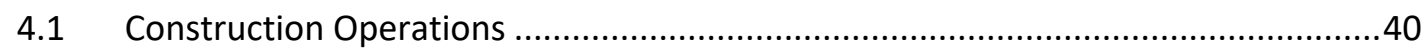

4.1.1 Case Study: Rock River Project.............................................................40

4.1.1.1 Project Background and Context..........................................40

4.1.1.2 Interactions of the Project ........................................................43

4.1.1.3 The Driving Interaction and Disequilibrium ..........................44

4.1.1.4 Complexity Analysis .........................................................44

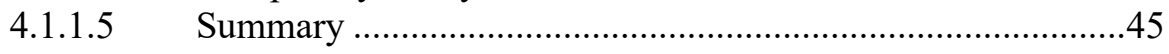




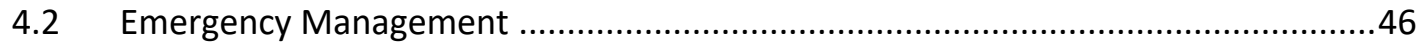

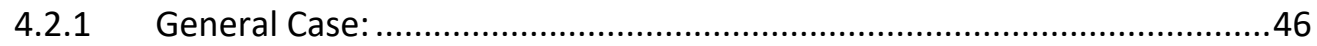

4.2.1.1 ICS Structure and Scaling ..................................................46

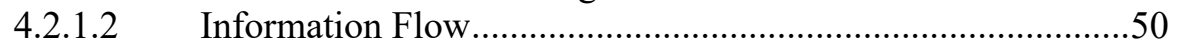

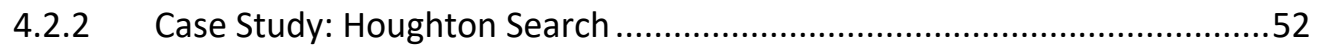

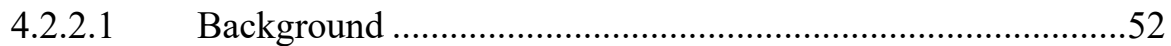

4.2.2.2 Search Operations ..............................................................53

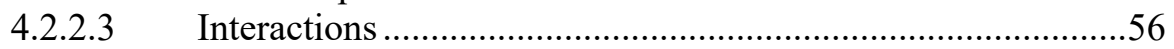

4.2.2.4 Areas of Disequilibrium .........................................................5

4.2.2.5 Measurement of Complexity …............................................59

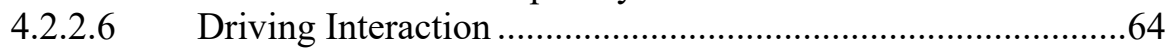

4.2.2.7 Summary ..........................................................................65

4.2.3 Case Study: 2018 Western Upper Peninsula Flooding ..............................66

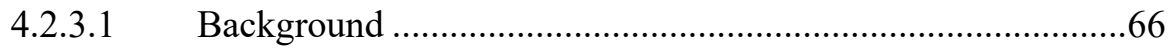

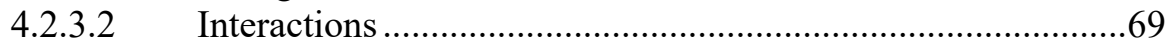

4.2.3.2.1 Initial Phase Interactions: .............................69

4.2.3.2.2 Stabilization Phase Interactions:......................70

4.2.3.3 Areas of Disequilibrium ......................................................72

4.2.3.4 Measurement of Complexity ……….....................................74

4.2.3.5 Driving Interactions.......................................................... 75

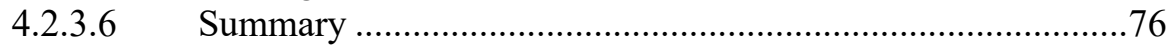

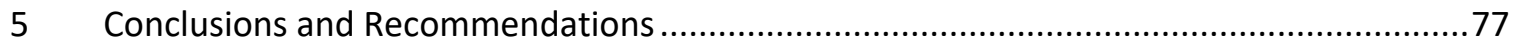

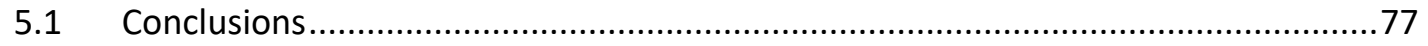

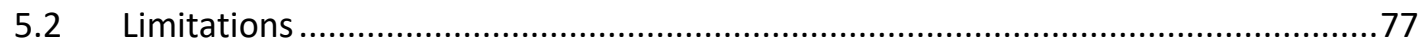

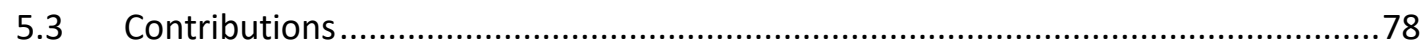

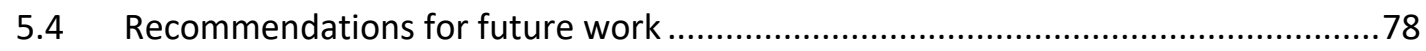

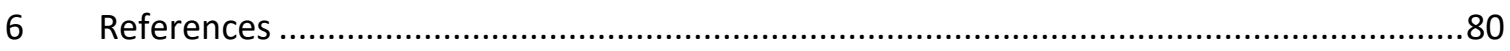

A Appendix: Additional Plots of Varying Frequency and Delay .........................................86

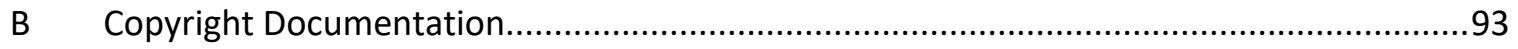




\section{List of Figures}

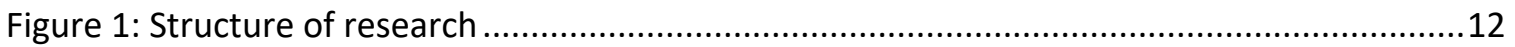

Figure 2: Three component construction cycle …............................................................. 15

Figure 3: Phase diagram of paving construction example .......................................................18

Figure 4: Example with an excavator and trucks. The excavator requires multiple cycles to load a

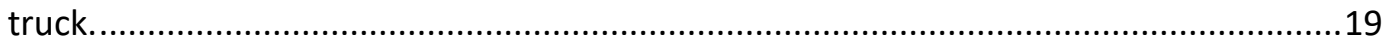

Figure 5: Balanced circle representation of excavator and truck interactions...........................20

Figure 6: Excavator/truck interactions with multiple trucks. ................................................21

Figure 7: Summation of truck/excavator interactions. The intersections between the circle and the hypocycloid are the interaction points between the trucks and the excavator................21

Figure 8: Adding a loader that takes the material from the truck to a batch plant......................22

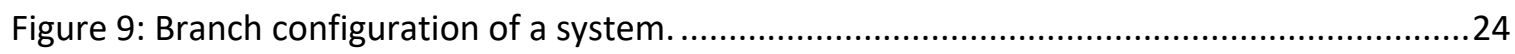

Figure 10: Nested circle diagram. Each layer represents a component interaction......................24

Figure 11: Unbalanced circle representation of truck and excavator interactions. ......................25

Figure 12: Example with an excavator and trucks. The excavator requires multiple cycles to load a

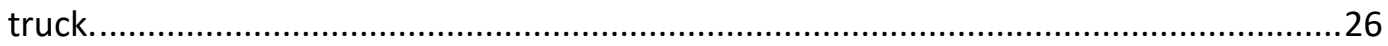

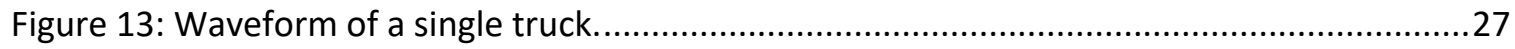

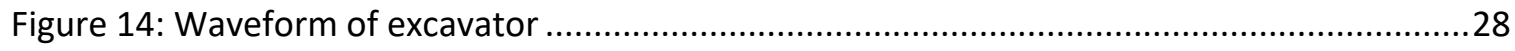

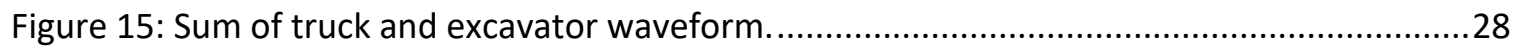

Figure 16: The sum of two components and the FFT magnitude and phase of the sum. .............30

Figure 17: Summation of three signals. The three frequencies are present in the second plot....30

Figure 18: Summation of 4 signals, the FFT magnitude, and phase ......................................... 31

Figure 19: Sum of very large numbers of frequencies ( $n=50,000$ random frequencies) and the FFT normalized magnitude showing the random frequencies used...................................... 32

Figure 20: Sum for very large number of frequencies ( $n=50,000$, linearly increasing) and the FFT normalized magnitude.

Figure 21: Two component signals with the excavator signal delayed by 0.025 34 
Figure 22: As delay increases, the FFT indicates additional frequencies present as harmonics....35

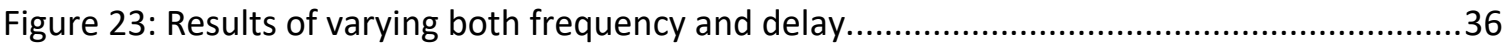

Figure 24: Phase diagram showing disequilibrium at the operating point.................................37

Figure 25: Interactions between contractor, owner, and sub-contractors ................................38

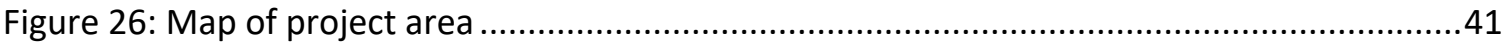

Figure 27: Interactions from the perspective of the bridge. ................................................... 43

Figure 28: Interactions without the rail line. The solid lines are primary interactions, and the

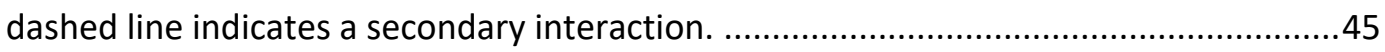

Figure 29: Generic Incident Command Structure ................................................................ 47

Figure 30: Example ICS structure for a single traffic stop incident ..........................................48

Figure 31: Example ICS structure for a multi-vehicle traffic accident.......................................49

Figure 32: Information flow for a single traffic stop incident. The delay introduced is the Information Request/ information Delivery feedback loop. ..........................................50

Figure 33: Information flow for multi-vehicle traffic accident ................................................51

Figure 34: Incident Commander/Unified Command Operational Cycle ....................................54

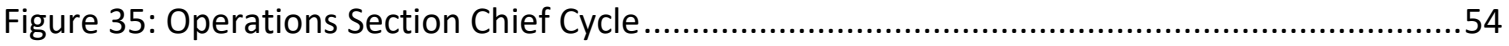

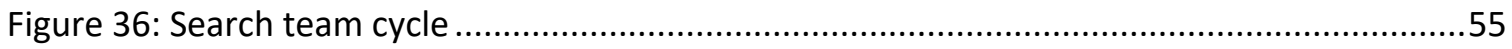

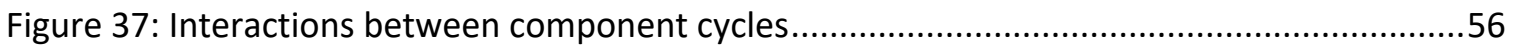

Figure 38: Initial operational period organizational chart ......................................................5

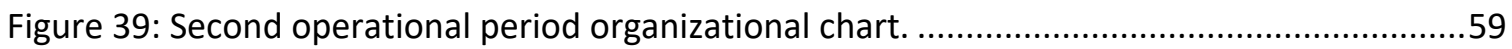

Figure 40: Information flow cycle diagram for a search. .........................................................60

Figure 41: Circular representation of the search operations for a balanced system, showing the various levels of organization and the multiple Search Teams and members. ................61

Figure 42: Unbalanced search circular representation of the search system. ............................62

Figure 43: Component waveforms from Houghton Search.....................................................63 
Figure 44: Summation of the four SAR component waveforms and the FFT of the sum, showing the magnitude and phase angle.

Figure 45: Second operational period (after additional staff added) organizational chart..........65

Figure 46: 6-hour Precipitation total: 1AM-7AM, June 17, 2018. Source: NOAA ........................66

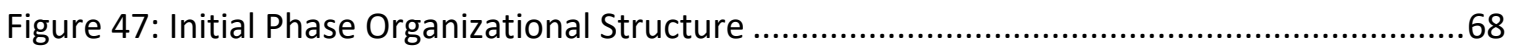

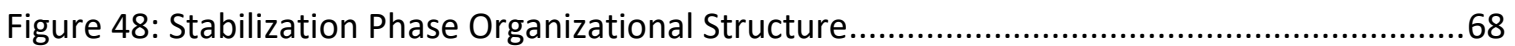

Figure 49: Unified Command-Agency Interactions during the initial phase of the 2018 flood. Note the overlap of the inner circles indicates disequilibrium................................................74

Figure 50: Unified Command-Agency Interactions during the stabilization phase of the 2018 flood

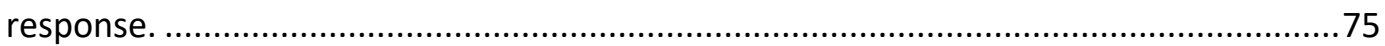

Figure 51: Varying the frequency and comparing the summation waveform, FFT magnitude, and phase calculations shows that each FFT magnitude plot has the frequency components clearly visible as spikes on the normalized magnitude plot. The phase angle of each component is plotted as well as comparison to the base case (left side).

Figure 52: Continuation of figure 48: Varying the frequency and comparing the summation waveform, FFT magnitude, and phase calculations shows that each FFT magnitude plot has the frequency components clearly visible as spikes on the normalized magnitude plot.

Figure 53: Plots of varying delay while holding the frequencies constant. The plots on the left are with no delay (balanced system). The plots on the right are with a delay of $\varepsilon=0.025$. The phase angle difference plot on the bottom is the difference in phase angle from the control (plot with $\varepsilon=0$ ) and this iteration (plot with $\varepsilon=0.025$ ).

Figure 54: Continuation from figure A-3. Continuing to increase the delay while holding the frequencies constant. The plots on the left are with a delay of $\varepsilon=0.05$. The plots on the right are with a delay of $\varepsilon=0.1$. The FFT in plot $(3,2)$ shows that the delay in the excavator plot is growing large enough that it effectively acts as another frequency in the system.

Figure 55: Varying both the frequency and delay and comparing the result to the baseline with two frequencies and no delay.

Figure 56: Continuation of figure 54. Varying both the frequency and delay and comparing the result to the baseline with two frequencies and no delay. .92 


\section{Abstract}

This research discusses the characteristics that make projects difficult to manage. Project inefficiencies and failures can be attributed to the structure of a system. Developing a measurement for complexity based on the number and nature of interactions in the system can allow project complexity to be reduced while still meeting project objectives. Previous research has identified characteristics or markers of complex systems but does not address how to measure or how to reduce a system's complexity.

The objective of this dissertation is to develop a metric for complexity that assists project and emergency managers in reducing project complexity either by engineering complexity out, if possible, in the planning stage, or by managing the system during an ad hoc incident. The proposed measure uses the number of interactions for a given perspective of the system as the metric of complexity and uses disequilibrium from the expected normal state as an indicator of an irreducible interaction and an indicator of complexity. The proposed hypothesis is discussed through mathematical and visual examples to illustrate disequilibrium in system interactions.

The methodology is demonstrated in three case studies: one in construction, through the Morgan Street Bridge project over the Rock River in Rockford, Illinois; and two in emergency response incidents (one local and one State/Federal). These cases demonstrate how this measure of complexity can be used to reduce complexity in a project by identifying the perspective of interest in the system, determining the disequilibrium in the interactions, and reducing the interactions while minimizing information loss. 


\section{Introduction}

\subsection{Problem Statement}

Project management, which includes emergency response, is fundamentally the management of a system of resources to accomplish project objectives (ICCPM 2012). Failures of project management reduce efficiency, increase cost, and can prevent the project objectives from being met (Jordan et al. 2016). Nationally, regionally, and locally, there is interest from emergency managers, responders, and elected officials to understand why communications continue to be a problem, especially given the large sums of money routinely allocated to improving communications and information flow (Comfort et al. 2004; Manoj et al. 2007). Therefore, the emphasis of this research is to explore the issues of information flow failures in project management - emergency response in particular. The question this research attempts to answer is: What specific characteristics make projects difficult to manage?

Project management has traditionally used methods that assume projects to be static, with fixed material quantities, and predictable timetables (ICCPM 2012). Variations to these assumptions are accounted for by incorporating contingency factors based on statistical characterization of specific variables related to cost and schedule. However, if the conditions of the project vary beyond this contingency envelop, projects begin to run "over budget" and "behind schedule" despite the best efforts of traditional project management. More recently, the complexity of projects has been recognized as the causal factor behind project management failures (ICCPM 2012), (Vidal et al. 2011). Vidal et al. (2011) states that "ever growing project complexity is an ever-growing source of project risks". The International Centre for Complex Project Management Standards (2012) classify projects as complex if the project is: emergent, adaptive with sensitivity to initial conditions, and contains feedback loops (ICCPM 2012); characteristics that are also supported by Remington and Pollack (2007) in their study of system complexity. It has been suggested that a pathway to improving efficiency, and reducing delays in project management, is to assess and address the challenges presented by complexity of projects (Owens et al. 2012; (ICCPM 2012).

Emergency management is a subset of project management. In the United States, the Federal Emergency Management Agency (FEMA) recognized the impact of poor project (or incident) management in the 1970's and began to put in place an organizational structure (called the Incident Command System (ICS) when it was finally implemented by the Homeland Security Act of 2002 (Nicholson et al. 2006)) to standardize emergency response in the United States (Bigley and Roberts 2001). One of the initial concerns was the ability for a standardized organizational structure to scale possessing the ability to work for incidents as small as a single car traffic stop all the way up to a hurricane response (Favero 1999). However, there continue to be failures even with this standardized Incident Command System in place. After Action Reports (AAR), emergency management practitioners, and the literature (Grinter et al. 1999) frequently identify information flow failures as a source of incident response inefficiency (Comfort et al. 2009; Federal Emergency Management Agency 2018a; Richardson and Byers 2006). 
Few failure modes of emergency response information flow have been identified (Manoj et al. 2007) and modern crisis are becoming more complex (Weick et al. 2008). However, literature reveals some clues as to where information flow failures have occurred. The work by Comfort et al. (2004) identified how the amount and type of information provided to responders impacts the emergency response efficiency. Comfort et al. (2004) also examines the structure of the response. Specifically, local jurisdictions respond more efficiently on their own, rather than in a centralized (state-wide for example) controlled system. However, the study did not examine the integrated response framework of ICS and the information flow through its structure, but rather a non-ICS structure. Another study by Jensen and Jr., Waugh (2014) suggests the ICS framework is not useful as an all-hazards framework but only in 'routine' or 'practiced' emergencies where responders already understood the structure of the response, suggesting that the structure of the response impacts the information flow. This result also indicates that when an incident is not a normal incident or does not progress as expected, there are information flow issues. Moynihan (2009) identifies a number of issues with the ICS framework's structure that are actually information flow issues. But the previous works do not identify the intersections between the information flow failure types with incident hazard type and size. Nor do these works directly address the issue of emergency response complexity, but they do discuss issues that are characteristics of complex systems. The conjecture is that the causes of information flow failure identified in the literature are actually indications of complex systems.

\subsection{Objective}

The objective of this research is to determine a measure of complexity and system characteristics that identify information flow failure in project management. This will determine the impact of system complexity on information flow. The result of this research will be a metric to assess complexity of systems and the likely points of failure. Emergency managers, responders, and project managers can apply this metric to engineer out or reduce the frequency and mitigate the impacts of information flow failures.

Previous works have examined various parts of complex systems and the emergency response process. Emergency response and recovery is a process involving many agencies, resources, and levels of government that must communicate to be successful and efficient (Jordan and JavernickWill 2013). Hence, the questions motivating this research are:

- System Complexity: What are complex system characteristics? How is complexity defined? How is complexity measured?

- ICS Structure: What is the structure? Are there known problems with the structure? How does ICS scale?

- Information flow: What information flow problems does ICS have? Does information flow scale as ICS scales? How does scaling change the information flow?

This dissertation specifically examines the case of information flow failures between components of a system through the lens of complexity. 


\subsection{Contributions and Expected Outcomes}

The contributions of this dissertation are the methods to identify the ways complexity is impacting projects in an objective way, in contrast to existing methods that are superficial and based on categorization. The development of a metric for complexity will contribute to identifying information flow failure points in project management and emergency response. Managers and coordinators can use this information to design their plans to limit the factors that negatively impact the information flow.

The expected outcomes of this research are:

- A method of measuring complexity

- Methods to identify disequilibrium in systems

These outcomes will allow managers to reduce the information flow failure potential of their projects.

\subsection{Organization}

The problem driving this dissertation is project management failure - why are failures occurring? The hypothesis is that project management failures are occurring due to the structure of the project system, specifically number and nature of the interactions between the components of the system. In order to test this hypothesis, a metric is needed to measure the complexity of a system.

This dissertation is organized into the following chapters:

Chapter 1 discusses the motivation in determining a metric for complex systems, states the objectives, and outlines the expected outcomes.

Chapter 2 discusses the background in complexity and reviews the literature of complex systems, disequilibrium, and discusses previous work to measure complexity. The points of departure from the literature are discussed leading to the proposed measure of complexity.

Chapter 3 investigates the number and nature of complex systems through a discussion of visualization and analysis methods. These methods include graphical representation of systems (project), mathematical representations, and then examines these representations using signal processing techniques to try and reveal the underlying structure of the system.

Chapter 4 applies the methods from chapter 3 in case studies. Three case studies are used (one in construction, two in emergency management) to show how the measure of complexity and the visualizations developed in Chapter 3 can be used to measure complexity, determine the disequilibrium in an interaction, and how complexity of the system can be reduced. 
Chapter 5 discusses the conclusions of this dissertation, discuss the limitations, and makes recommendations for future research.

Next, this dissertation will review the literature and determine the points of departure for this research. 


\section{Background}

\subsection{Definitions}

This dissertation will address systems, project management, complexity, and information flow. While the definitions of these terms vary throughout the literature, this dissertation will use the following definitions for consistency:

Systems are defined as individual components connected together to function as a single unit. These connections are the internal interactions of the system. Systems can be permanently connected together or can be formed ad hoc. The whole system can be altered by changing out one component (ICCPM 2012).

Project management is defined as the organization and coordination of resources to accomplish a set of agreed upon objectives (either contractual or goal driven). Emergency response is a subset of project management because it also requires coordination and assignment of resources to form a system to accomplish objectives (ICCPM 2012).

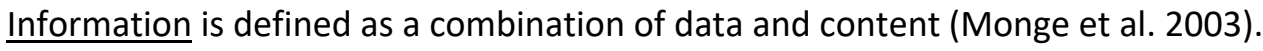

Information flow is defined as the movement of a specific volume, content, or frequency of information between components of the system within a given time period (Matisziw et al. 2008). However, unlike a tangible object, the movement of information does not remove it from a previous location (Leonidas 2011).

Information flow failures occur when the information is not completing the path between two components of the system. The failures are occurring at the information exchange points where two parts of a system are connected - i.e. the interactions in the structure of the system. Zachary (1977) also describes an information flow failure as a "bottleneck" or structural limitation.

A channel is the path information flow takes. This could be in the form of voice traffic or electronic information (such as an email) (Zachary 1977).

It is also important to note the differences between project operation, organization, and management.

Project operation refers to the cyclic tasks to accomplish project objectives or sub-objectives.

Project organization refers to the system that defines the relationships between the components.

Project management is defined by (Munns and Bjeirmi 1996) as directing tasks and resources to accomplish the project objectives. 


\subsection{Defining Complexity}

Monge et al. (2003) discuss complexity in communication networks through a review of complexity and modeling research. Monge et al. (2003) describe the components of complex systems as a network of agents, agent attributes and traits, rules for agent interactions, and the structure that results from the agent rules and interactions. Describing complex systems as a network of entities, Brook (2016) and Monge et al. (2003) also includes the topological relationships between agents in the network impacting how agents transfer information between each other. Holden et al. (2013) agree with the importance of complex network topology but look at it from a resilience to disruption perspective. However, the number of relationships (connections) is not discussed further in these works. In similar work, Provan and Kenis (2008) discuss governance (control and organizational) for complex networks but do not clearly define what "complex" means. In addition to traits like experience and expertise (among others), three categories of traits by Axelrod et al. (1999), location, capabilities, and memory, are discussed. Key among these is memory, which includes past experiences, known contacts, and where agents can get information. Manson (2001) notes that the linking between components in a complex system allows them to accommodate a wide range of situations, in part, due to this memory characteristic, and Phelan (1999) states that agents learn from their own localized experiences. From a project management and emergency response perspective, the memory trait is important for an efficient response and forms the basis of why responders participate in training and exercises - to build that memory and experience set, when real events may be infrequent (Federal Emergency Management Agency 2020). The rules governing interactions prescribe how an agent interacts with others (Little 2003; Monge et al. 2003). These rules are not necessarily static and Monge et al. (2003) even proposes that they are not rules but meta rules, where agents choose to follow or change the rules depending on the circumstances. Similar comments have been made by Gideon et al. (2005) about components following rules and practices laid out by management in order to become part of the larger system. Stochastic behavior of agents, such as deciding to follow rules or not, is beyond the scope of this research. This research will focus instead on events with a predetermined structure such as the incident command system (ICS), due to the requirement for emergency response to follow the ICS structure per Federal Emergency Management guidelines. However, it is noted in literature (Moynihan (2009) and (Bennet 2011) for example) that responders do not always adhere to this requirement.

Crutchfield and Young (1989) define simplicity of a system as the ability to mathematically reduce its behavior without information loss. In their definition, simplicity does not vary linearly with the level of information content in the system. Systems that exhibit periodic behavior are considered to be just as simple as systems that exhibit truly random behavior because in both cases the systems can be mathematically represented using periodic and stochastic equations, respectively, and therefore can be reduced without any information loss. Hence, for both periodic and stochastic systems the information content is considered to be low, while the information content is considered to be higher for systems exhibiting not truly random behavior. Hence, their definition of complexity is a measure of the inability to reduce a system mathematically without loss of information. Such complex systems tend to be in between the extremes of periodic systems and systems that exhibit true random behavior, because they cannot be mathematically reduced without information loss using either periodic or stochastic equations. These systems are 
often considered to be chaotic and of medium information content. Formally, Crutchfield and Young (1989) define complexity as:

"The ... approach is an abstract notion of complexity: the information contained in the minimum number of equivalence classes obtained by reducing a data set modulo a symmetry."

"The equivalence classes" represent the reducible information in the system. This may reflect repetitive patterns in the structure of the system. The "symmetry" is the expected or standard reference value. Hence, a standard pattern may be considered to be a symmetry. A simple system could then be reduced to an exact integer number, say $n$, of the pattern without any information loss. However, if the system has information that is lost in representing it using $n$ patterns, then a measure of the lost information, or the remainder of the system after the symmetry is removed (i.e., the "modulo") may be considered to represent a measure of its complexity. This concept is echoed by Bertelsen (2003) who states complex systems cannot be completely reduced without information loss. The modulo, hence becomes, the indication of complexity from the perspective of the chosen symmetry. In this interpretation, the measure of complexity of a system is sensitive to context, as the choice of symmetry can change the reducibility of a system. Typically, the symmetry value may be based on prior data, field standards, or experiences. The choice of symmetry also defines the perspective of inquiry, meaning the reducibility of a system is predicated on the area of interest. This idea is also stated by (Gell-Mann and Lloyd 2004) who noted that complexity is subjective to context.

In related literature, López-Ruiz et al. (1995) discuss the concept of "disequilibrium" as a measure of the distance from the mean state. As a system diverges from the expected point of stable equilibrium, the disequilibrium increases, indicating an increase in the measure of complexity in the system. This idea echoes the discussion of complexity as per Crutchfield and Young (1989). The system modulo a given symmetry is analogous to a deviation from a point of equilibrium, and the remainder value is analogous to the extent of the deviation. Hence, the difference, as well as the extent of disequilibrium, can be used as an indicator of complexity. This leads to the question of "How much disequilibrium or deviance is too much before the system either collapses or becomes too much of a risk?" (Hall 2003). Bertelsen and Koskela (2005) describe the disequilibrium as a variance - a cause for concern for part manufacturing but using the same ideadisequilibrium causes uncertainty. System resilience, robustness, and risk are not addressed in this dissertation but recommended for future work.

Construction operations can be represented using activity cycle diagrams due to their inherent repetitive nature. At the project level, linear and repetitive projects can also be represented as a sequence of repeating activities based on unit production rates. These repeating patterns provide the ability to select meaningful equivalence classes and symmetries. Context will drive such a choice based on whether the inquiry is at the operation, project or management level (Gell-Mann 2002). A question such as "Is this a financially complex project?" might indicate one result, but for the same project, "Is there scheduling complexity?" might yield a different result. The idea of context is echoed by ICCPM (2012) who described the problem as using "multiple metaphors and dialects" to obtain different perspectives on the problem. 


\subsection{Foundational Work: Measuring Complexity}

Previous works on system complexity have focused on posterior assessment and the classification of projects as "complex" or "not complex". There is, however, a need to define and develop a metric for complexity based on an identification of factors that directly contribute to project complexity (Gidado 1996). Without such a metric, it is not possible to reduce system complexity or engineer a system to be more resilient to complexity.

Previous research (Dao et al. 2016; Owens et al. 2012) has used the Delphi method to rate and differentiate between the extent to which factors, such as cost, design, project context, project finances, infrastructure size, infrastructure interconnectivity, infrastructure newness, and project uncertainty, contribute to project complexity (Safapour et al. 2018). In their approach, using the highest ranked factors, Owens (2012) selected and investigated five case studies by interviewing the project participants involved. The research by Owens (2012) and additional research by Vidal et al. (2011) identified factors that tend to correlate with, or are markers of, complex projects. While the results of this research can be used to rate the complexity of a project based on a list of criteria, they stop short of directly identifying a general set of conditions and project characteristics that give rise to complexity. The recommendations also provide limited guidance on ways to re-engineer projects to reduce the complexity at the source. The qualitative nature of the study and the generalization from expert opinion based on five case studies limit the longterm applicability of the research as innovation and technology continue to shape project means and methods.

Remington and Pollack (2007) identified characteristics of complex systems as hierarchy, communication, control, emergence, phase transition, non-linearity, adaptiveness, and sensitive dependence on initial conditions. A discussion of these characteristics follows.

Control is defined by Remington and Pollack (2007) as the ability of the overall system to maintain order and carry out the overall objectives - even with the individually linked yet semi-autonomous units operating simultaneously. Whitty and Maylor (2007) discuss the difficulty of controlling projects when individual agents are interacting but are simultaneously dynamically changing. Gransberg et al. (2013) noted that as complexity increases the ability for a project manager to maintain control decreases in complex projects.

Emergence occurs when the level of interaction among the components of a system reaches a critical point and the overall system accomplishes tasks that requires the entire system (i.e. not by any individual components) (Remington and Pollack 2016). These tasks may or may not be the intended objective of the overall system or may or may not even be productive for the system. Multiple sources commonly refer to this by saying "the system is greater than the sum of its parts" (Remington and Pollack 2007, Whitty and Maylor 2007, Paina and Peters 2012, Gideon et al. 2005, Manson 2001). Whitty and Maylor (2007) note a lack of sufficient tools to determine what interventions that managers could take to be effective in complex projects to compensate for unintended emergent issues.

Phase transition is described as a "radical" change in the form of operation of the same system components to accommodate changing parameters (Remington and Pollack 2007, Eusgeld et al. 
2011, Paina and Peters 2012). This varies from adaptiveness in that it is not changing the internal components (or agents) but is reconfiguring them to perform a different function. Adaptiveness, in contrast, may change the internal components to improve the overall system to meet new objectives (Whitty and Maylor 2007). However, Eusgeld et al. (2011) noted tools are lacking to analyze and model these adaptive systems.

Non-linearity is a result of feedback to the system from other system components (Remington and Pollack 2007, Eusgeld et al. 2011). The information gained through dynamic feedback loops influences the components of the system and provides the basis for the adaption or phase transitions that may take place. The communication is the medium through which the data moves, but the dynamic feedback contains the information that can reinforce plans or alter the direction of the operation (Paina and Peters 2012). This concept is reinforced by Murray et al. (2007) who found a greater number of interactions (communication) does not automatically mean greater connectivity (useful data). Unaddressed in the work by Murray et. al (2007), is the lag time in feedback loops and their impact on the overall system.

The organizational hierarchy of complex systems is often described as a system-of-systems whereby each individual sub-component can (normally) operate independently but also have the ability to combine into a complex system when needed (Gideon et al. 2005; Remington and Pollack 2016). Using smaller units of operation, large tasks can be broken into manageable pieces and distributed. One area not addressed in this definition is the diminishing returns of distributed work processing, i.e., addressing the balance point between parallel processing of tasks verses the overhead needed to manage all of the agents. Gideon et al. (2005) has developed a taxonomy for systems-of-systems based on their design, operation, and domain.

The International Centre for Complex Project Management Standards (2012) classify projects as complex if the project is emergent, adaptive with sensitivity to initial conditions, and contains feedback loops (ICCPM 2012); characteristics that are also supported by Remington and Pollack (2007) in their study of system complexity. It has been suggested that a pathway to improving efficiency and reducing delays in project management is to assess and address the challenges presented by complexity of projects (ICCPM 2012; Owens et al. 2012).

Complex system characteristics, specifically feedback systems, organizational hierarchy, and control are examined through the lens of communication. Communication channels are the links between the components of a system. System performance is an indication of the strength of those links. Matisziw et al. (2012) examined the structure and performance of a network as a measure of network robustness. They determined that nodal interactions, when dynamic, significantly impacted the robustness of a network. This is because certain edges or nodes in a network have higher importance and therefore have a higher impact on the stability of the network (Matisziw et al. 2010). Their objective was to identify locations on the network that could be reinforced and fortified to improve its survivability in the event of an attack. From a volume of information perspective, Miller (1956) found that an individual's maximum manageable interactions is "about" seven. Flood (1987) calls this the "threshold of complexity". FEMA (2018) calls this the "span of control" and suggests that it be limited to 5 individuals or resources for every 1 supervisor. To compensate beyond this threshold, Miller (1956) found that people begin to chunk information or package it into larger pieces, often with less detail. Further work is needed 
to examine the impact of this information chunking. Further work should examine the rate of information flow between the manager and multiple individual agents and how the cycle of information gathering and sharing is impacted. There is an interesting parallel with work done by Payne (1976) regarding decision making by individuals. That work noted that in a complex situation, decision makers used an "elimination by aspects" to filter out alternative options in order to make a decision. This is effectively discarding information intentionally when there is too much information to process.

Attempts at measuring complexity in health care system scaling research have compared crosssectional case studies. Their objective is to use internal system dynamics as a method of identifying critical management points, scenario planning and exercise activities with dynamic rules (Paina and Peters 2012). The results indicate that dynamic interactions, stakeholder participation, and local conditions were all important components in the scaling of health systems. McCabe (1976) measured complexity in computer programming as the number of potential paths a program can follow using graph theory. Although feedback loops are accounted for in this metric, the "interactions" between agents is limited or fixed. Attempts to measure system complexity have also included using statistical methods using entropy as a model (LópezRuiz et al. 1995). They discuss the idea of a "disequilibrium" as a measure of the distance from the probable likely (mean) state. This is multiplied with an information variable (representing information stored in the system), to give a measure of complexity. As an example, if the likely probable state has a disequilibrium of zero, the complexity of the system is zero (López-Ruiz et al. 1995). This approach does not account for the number of interactions of the system specifically. Gidado (1996) developed a measure of complexity but limited it to the length and cost of a project and did not address other objectives. The Gidado (1996) measure was based on the number of components and the duration of the workflow. Finally, Austin et al. (2002) discuss the advantage of using graphical models to visualize complexity, noting that graphical models can lead to a greater understanding of the information flow in a system.

While reducibility of projects has been discussed in this research, as a way to reduce failures of information flow, there is a tension between the goals of reducing project complexity and still maintaining a robust system that is capable of adapting to component failures. Research has been conducted by Gribble (2001), lyer et al. (2013), and Paul et al. (2004) examining the robustness of networks when nodes or edges are removed and examine the ability of the network to continue to function. This work identifies critical nodes or edges which, if removed, causes network failure. Using the research from this dissertation, the previous work leads to the questions: is it better to have a less robust system (more prone to failure) or a less complex network, less prone to disequilibrium (delay, information loss) and failure? Does a robust network require complexity? It is important to note that much of the network research is based on infrastructure or social networks, that do not have a "project" objective the network is "trying to accomplish". Therefore, the failure of a "node" or component in a project system does not necessarily preclude another component from taking over the vacant role (i.e., if the project manager is suddenly incapacitated, a subcontractor might be able to step in and conduct that role).

The properties of the system allow for the failures in information flow. These include, but are by no means limited to: 
- Too much information for the channel to accommodate, or the receiver to process (Miller 1956)

- Not enough information is available for the receiver to make use of the information (Comfort et al., 2004)

- Delay in the system and the information does not arrive in an acceptable timeframe to be used as desired (Moynihan 2009).

\subsection{Points of Departure}

A summary of the points of departure from the literature are:

- Previous works identify indicators of complexity but not metrics to quantify a system's complexity.

- Complexity research has tended to use Delphi approaches (reliance on experts) rather than qualitative measures. This limits the scope of the research use to specific fields. It also potentially limits the application as it provides no direction on how to reduce complexity in the system.

These points lead to the following research questions:

- How is system complexity defined and measured?

- Can information flow failures be related to changes in complexity?

\subsection{Proposal}

To answer these questions, this dissertation proposes to develop a metric of complexity that provides a quantitative metric and provides guidance on how to reduce complexity.

The hypothesis of this research is that complexity is a characteristic of the organizational and operational structure of an event (i.e., a project or incident) based on the number and nature of interactions within the system.

This hypothesis is tested across both hypothetical and real-world events to support the creation of a metric that measures the system complexity. Based on a review of definitions of complexity in mathematics and other inter-disciplinary bodies of systems literature, this research devises a definition that can be used in different contexts to characterize the complexity of a project or event. Based on the definition by Crutchfield and Young (1989), this research recognizes that the answer to "how complex is a project?" also depends on the context within which the question is posed. For instance, a project with multiple interacting operations and schedule dependencies may not have the same level of complexity in its financing and delivery methods. However, this dissertation posits that the complexity of each of these contexts can be characterized using identical systems and mathematical constructs of equilibrium and phase change. Based on these ideas, this research demonstrates that the number and nature of critical interactions within a system is a good measure of the project complexity, given a context specific construct. In turn, this number provides a simple metric that can be used by practitioners to identify interactions that increase the complexity in a project and engineer ways to reduce the project complexity. The 
dissertation concludes with several case studies identifying complexity as a function of interactions between project components. The case studies are analyzed through the framework this dissertation developed to measure and reduce complexity.

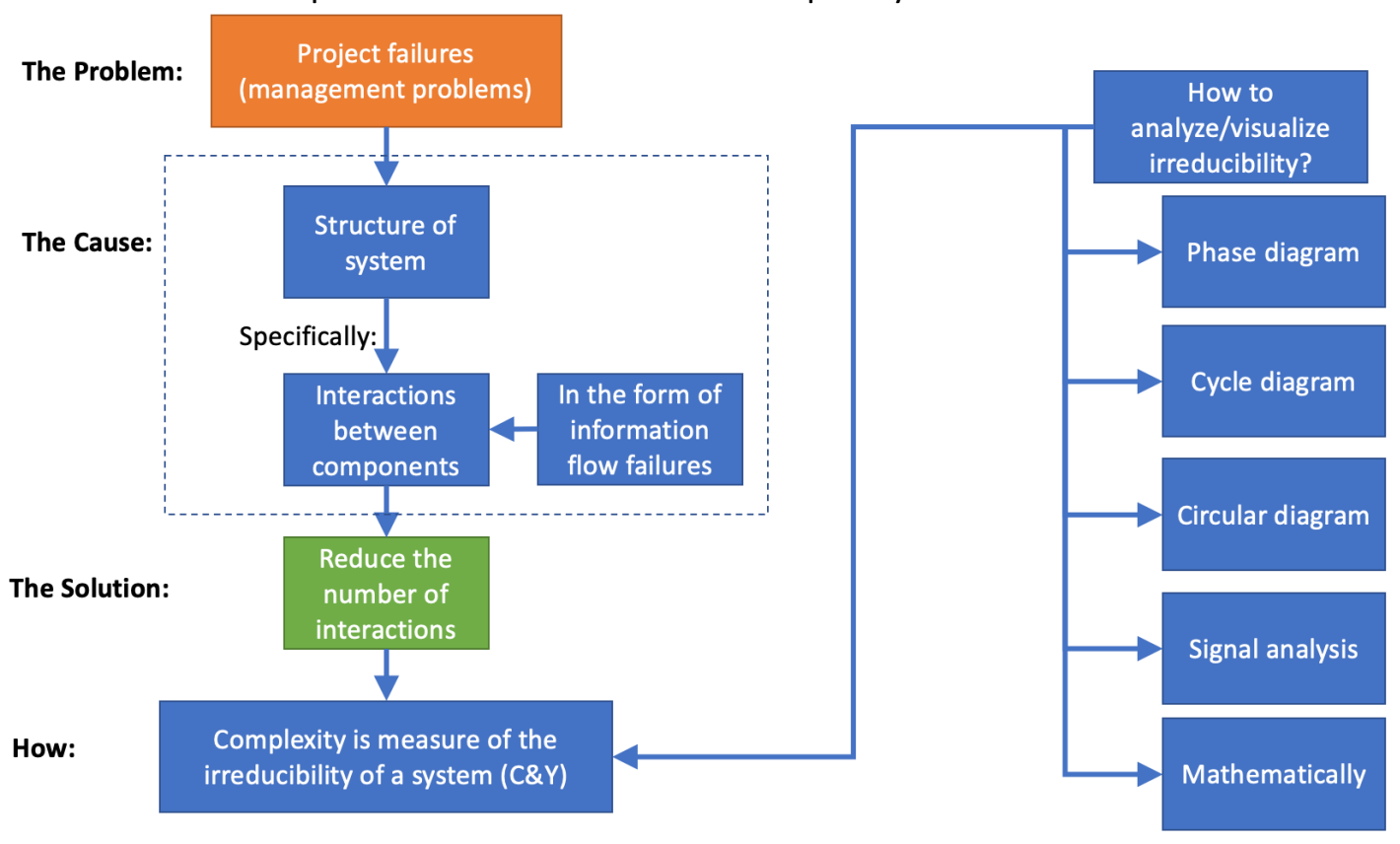

Figure 1: Structure of research

Figure 1 outlines the structure of this research. The first step in testing this hypothesis is to identify methods of representing a system that accounts for both the number and nature of system component interactions. Both visual and mathematical representations will be use and are discussed in Chapter 3. 


\section{Building Blocks of Complexity}

This chapter will develop the methods of representing systems for analysis. Based on the literature, two of the parts of complexity are of particular interest, component interactions and disequilibrium which will be discussed as the number and nature of the interactions. Determining when there is disequilibrium and where component interactions occur is important in determining a system's complexity as it highlights what is making a system complex.

Systems are built from individual components that interact to complete the system task. Identifying the component interactions from the overall system behavior presents challenges as the component interactions are combined and may appear as one from outside the system. These interactions are the points of information transfer within the system. Given the objective of this dissertation is to determine the reason for information flow failures, identifying the transfer points of information is a critical step in identifying points of failure.

Disequilibrium refers to the difference between a balanced and an unbalanced component interaction. In physical systems, this can be manifested as a delay or a "waiting" for one component to finish a task before the next task can begin. As mentioned in Chapter 2, López-Ruiz et al. (1995) discuss the concept of "disequilibrium" as a measure of the distance from the mean state. As a system diverges from the expected point of stable equilibrium, the disequilibrium increases, indicating an increase in the measure of complexity in the system. This idea echoes the discussion of complexity as per Crutchfield and Young (1989). The system modulo a given symmetry is analogous to a deviation from a point of equilibrium, and the remainder value is analogous to the extent of the deviation.

In construction project management, the use of equilibria or balance-based principles is not uncommon (Yang and loannou 2001). Hence, combining the ideas from Crutchfield and Young (1989) and López-Ruiz et al. (1995), it is conjectured that the concept of disequilibrium can be used to indicate system complexity in that interaction. A system in disequilibrium is more irreducible given a symmetry, and the remainder - or the deviation from the equilibrium - can be considered to be an indicator of the complexity in the project component. The question at hand is: what are the factors that are likely to push a system out of balance or introduce a disequilibrium in a system? For example, in a simple construction operation design where the principle of continuous operation is used to balance production rates between equipment, balance is more difficult to achieve if there are more interacting cycles. Hence, the hypothesis is that an important factor contributing to the potential for deviations from the symmetry is the number of interactions within a system.

Given that both component interactions and disequilibrium are the components of complexity, measuring the disequilibrium and the interactions is important in determining a system's complexity. Since each point of interaction has the potential to be in disequilibrium, the more points of interaction there are, the more potential there is for disequilibrium. A key point of this dissertation shows: the more interactions, the more complex the system.

A goal of this research is to take the expressions of the principals of operations and use them to measure complexity. Delay and mismatch of resources are examples of disequilibrium and are all 
indications of complexity. A better understanding of complexity sheds light on operational efficacy. This is presented several ways: as signals, as a phase diagram, and by representing a cycle as a circle in the time domain.

Graphically, cyclic systems and cyclic system components can be represented as signals (waveforms) with a characteristic frequency that reflects the component behavior. Using a signal representation allows the system to be analyzed using signal processing techniques.

As the number of interactions increase, the number of degrees of freedom increase, which in turn, increase the possible states within which the system can exist. Thinking about this from the perspective of any given interaction between two equipment types in a system, each interaction has an expected pattern of behavior (the symmetry). Deviations from this expected behavior results in a disequilibrium (using López-Ruiz et al. (1995)) and can be measured, as the deviations are already quantitative in nature (e.g., dollars over budget, days over schedule, cubic yards of materials short, minutes spent waiting or idle). The proposed measure of complexity for this paper is to use the number of interactions for a given symmetry (perspective) of the project. The disequilibrium detected in the interaction is important, as the complexity arises from all of the ways in which a degree of freedom can vary and thus introduce uncertainty into the system.

In the next section, construction operation is mathematically analyzed to show that as the number of interactions increase, so does the number of points at which the system can deviate from a desired equilibrium. This dissertation discusses how this measure can be used at all project levels as a method for assessing project complexity and examines several methods of measuring the nature and number of interactions.

\subsection{Complexity in Construction Operations}

Operations with repetitive processes, like construction processes, can be reduced to cyclic interactions. Each cycle has an output metric (time, productivity, etc.). At the project level, linear and repetitive projects can also be represented as a sequence of repeating activities based on unit production rates. These repeating patterns provide the ability to select meaningful equivalence classes and symmetries. Context will drive such a choice based on whether the inquiry is at the operation, project, or management level. A question such as "Is this a financially complex project?" might indicate one result, but for the same project, "Is there scheduling complexity?" might yield a different result. The analysis of these cycles determines the balance point of the interacting resources. At this balance point, the driving design principle is that of continuous operation. Continuous operation reduces delays and wait times through the uninterrupted flow of resources. Continuous operation also implies the system is in equilibrium, or that there is a balance in production rates of equipment at each interaction point leading to zero waiting times. However, when the production rates of equipment are unbalanced, the waiting times can become non-zero and vary irregularly, causing the system to move into disequilibrium. For such a system, estimating the net production rate becomes less reducible due to the unexpected delays and waiting times. This increases irreducibility of the system. Therefore, by determining the extent of delay, or imbalance in the system, and the number of interactions in the system that can cause such imbalance (disequilibrium) and irreducibility, the level of complexity can be measured. 
Graphical models are used to visualize interactions and disequilibrium through theoretical examples. Real systems do not usually give off signals that can be measured, but the purpose of these graphical models and signal processing is to highlight methods to understand how systems work and components within those systems interact.

Consider the example of a hypothetical mainline concrete paving operation. A concrete paving machine is being serviced by trucks that are transporting the concrete mix from a batch plant to the paver. The batch plant is being serviced by water trucks delivering water. The water is crucial for maintaining a continuous supply of ready-mix concrete, necessary for the paver to function continuously. The activity cycle diagram for the operation is illustrated in Figure 2 . In one cycle the trucks deliver concrete from the batch plant to a paver (paver cycle), while in the other cycle the batch plant is supplied by trucks delivering water from a water source (water cycle). Ideally, the number of resources will be balanced so as to minimize idle time of all of the interacting equipment. In Figure 2, the number of trucks in the paver cycle is seen from two directions: the number of trucks needed to keep the paver continuously operating: $n_{0}$, and the number of trucks to keep the batch plant in non-stop production: $n_{1}$. The condition for the paver cycle to be balance is, $n_{0}=n_{1}$.

Time to travel: $t_{01}$

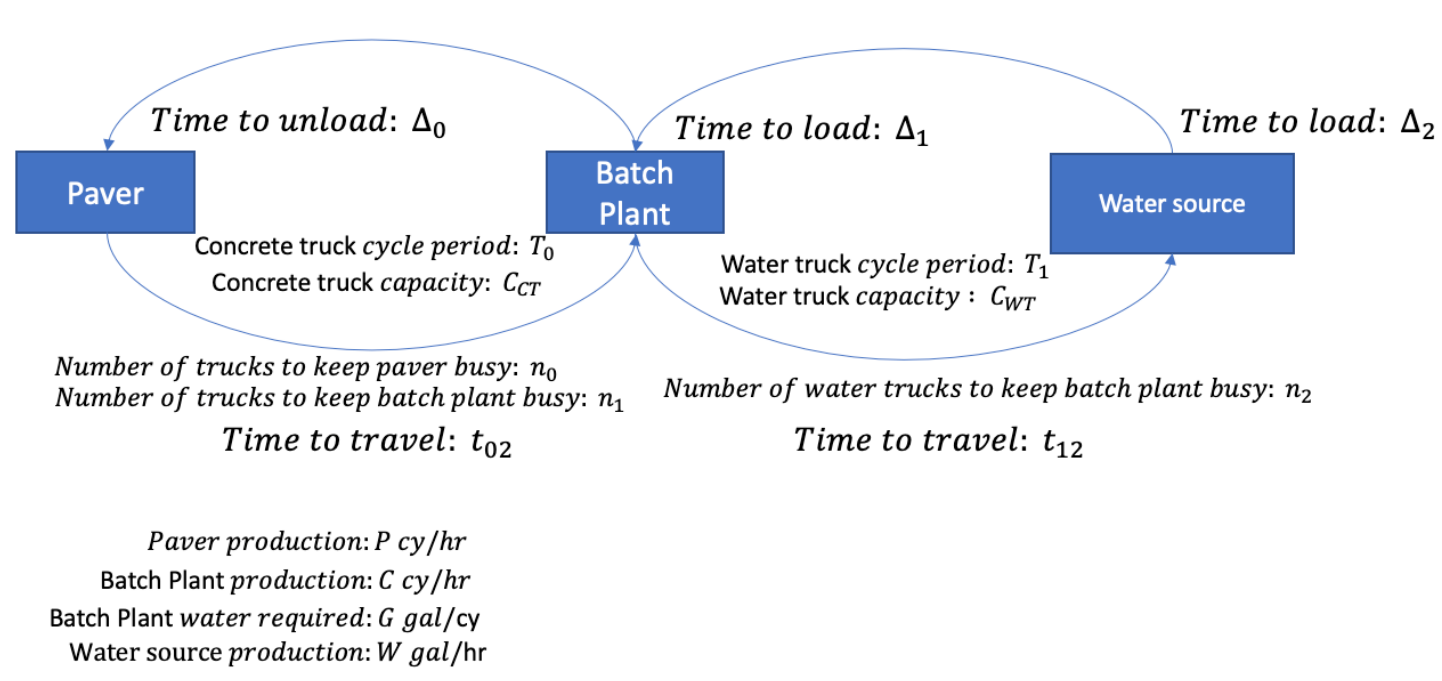

Figure 2: Three component construction cycle

This relationship will be the basis for analyzing the cycle. The paver production rate is denoted by $P$ (in cubic yards of concrete per hour), the batch plant production rate is $C$ (in cubic yards of concrete per hour), the water required in the batch plant is $G$ (in gallons per cubic yard of concrete produced), and the water supply from the source is $W$ (in gallons per hour). In addition, $n_{2}$, denotes the number of water trucks needed to keep the batch plant in continuous operation.

The number of water trucks needed $\left(n_{2}\right)$ to keep batch plant in continuous operation is:

$$
\mathrm{CG}=n_{2} \mathrm{~W}
$$




$$
n_{2}=\frac{\mathrm{CG}}{\mathrm{W}}
$$

If $C_{C T}$ is the capacity of the truck (in cubic yards) then the number of concrete trucks $\left(n_{1}\right)$ servicing the batch plant each hour to keep the batch plant in continuous operation is:

$$
\begin{gathered}
\mathrm{C}=n_{1} \mathrm{C}_{\mathrm{CT}} \\
n_{1}=\frac{\mathrm{C}}{\mathrm{C}_{\mathrm{CT}}}
\end{gathered}
$$

The number of concrete trucks $\left(n_{0}\right)$ needed to keep the paver in continuous operation each hour is:

$$
\begin{aligned}
& \mathrm{P}=n_{0} \mathrm{C}_{\mathrm{CT}} \\
& n_{0}=\frac{\mathrm{P}}{\mathrm{C}_{\mathrm{CT}}}
\end{aligned}
$$

Substituting for $\mathrm{C}_{\mathrm{CT}}$, from Eq. 4 in Eq. 6 :

$$
n_{0}=\frac{n_{1} \mathrm{P}}{\mathrm{C}}
$$

And substituting for C, from Eq. 3 into Eq. 2:

$$
n_{2}=\frac{\left(n_{1} \mathrm{C}_{\mathrm{CT}}\right) \mathrm{G}}{\mathrm{W}}
$$

If $n_{0}=n_{1}$, the balance condition for the paver loop, Eq. 6 in Eq. 8 can be substituted to get:

$$
n_{2}=\frac{\mathrm{PG}}{\mathrm{W}}
$$

Therefore, at the balance point for both the paver cycle and the water cycle the condition is:

$$
\begin{gathered}
\frac{n_{0}}{n_{1}}=\frac{\mathrm{P}}{\mathrm{C}}=1 \\
n_{2}=\frac{\mathrm{CG}}{\mathrm{W}}=\frac{\mathrm{PG}}{\mathrm{W}}
\end{gathered}
$$

At the balance point, the system is in equilibrium, and the expected production of the system is easily reducible from the primary production rates $P, G, C$ and $W$. However, if the system is not in 
equilibrium (i.e. $n_{0} \neq n_{1}$ ), delays are present in the system and there is a higher level of complexity as the number of variables cannot be related and reduced without knowledge of the extra variable $\mathrm{C}_{\mathrm{CT}}$. This increases the level of complexity in the system.

The equilibrium conditions as a function of the number of trucks (dimensionless parameters) can be analyzed as follows:

- If $n_{0}>n_{1}$, the paver production is greater than the batch plant $(\mathrm{P}>\mathrm{C})$. This is not physically possible (an inviable state) but is a mathematical solution indicating that the paver is idle.

- If $n_{0}<n_{1}$, the batch plant production is greater than the paver $(\mathrm{P}<\mathrm{C})$, and the batch plant is idle.

The summary of these results is listed in Table 1 . The production rates for the paver and batch plant are represented by $\mathrm{P}_{0}$ and $\mathrm{C}_{0}$ respectively.

Table 1: Production Rates

\begin{tabular}{|c|c|c|c|c|c|c|}
\hline $\begin{array}{l}\text { Paver } \\
\text { Production }\end{array}$ & $\begin{array}{l}\text { Batch Plant } \\
\text { Production }\end{array}$ & $\mathrm{n}_{0} / \mathrm{n}_{1}$ & $\begin{array}{l}\text { Idle } \\
\text { Equipment }\end{array}$ & $\begin{array}{l}\text { Production } \\
\text { Based on }\end{array}$ & $\begin{array}{l}\text { System } \\
\text { Interactions }\end{array}$ & $\begin{array}{l}\text { Batch Plant } \\
\text { Interactions }\end{array}$ \\
\hline \multirow[t]{2}{*}{$\mathrm{P}=\mathrm{P}_{0}$} & $\mathrm{C}=\mathrm{C}_{0}$ & $n_{0} / n_{1}>$ & Paver & $\mathrm{C}_{0}$ & 5 & 3 \\
\hline & & 1 & & & & \\
\hline \multirow[t]{2}{*}{$P=P_{0}$} & $\mathrm{C}=\mathrm{k}_{1} \mathrm{C}_{0}$ & $n_{0} / n_{1}<$ & Batch plant & $\mathrm{P}_{0}$ & 5 & 3 \\
\hline & $k_{1}>1$ & 1 & & & & \\
\hline \multirow[t]{3}{*}{$\mathrm{P}=\mathrm{P}_{0}$} & $\mathrm{C}=\mathrm{K}_{2} \mathrm{C}_{0}$ & $n_{0} / n_{1}=1$ & None & $\mathrm{P}_{0}$ & 3 & 2 \\
\hline & such that & & & & & \\
\hline & $n_{0} / n_{1}=1$ & & & & & \\
\hline
\end{tabular}

Table 1 also illustrates the possible states that the system can exist in. For this system, there are 5 interactions if $n_{0} \neq n_{1}$ :

- Trucks $\left(n_{0}\right)$ with batch plant

- Trucks $\left(n_{0}\right)$ with paver

- Trucks $\left(n_{1}\right)$ with batch plant

- Trucks $\left(n_{1}\right)$ with paver

- Batch plant and water truck

But, if $n_{0}=n_{1}$, the interactions reduce to 3 :

- Trucks $\left(n_{0}\right)$ with batch plant

- Trucks $\left(n_{0}\right)$ with paver

- Batch plant and water truck 
Therefore, by eliminating the delay (disequilibrium) in the system, the number of interactions is reduced. For a system, as the number of states increase, so do the degrees of freedom of the system, and therefore the complexity of the system increases from the batch plan perspective.

\subsection{Phase Diagram}

A phase diagram can be used to illustrate the possible states in which a particular interaction of the system can exist. In two dimensions, Figure 3 illustrates the phase diagram for the construction paving example showing the possible states in which the production rates of the paver, batch plant, and the overall system can relate to each other. Below the balance point $(\mathrm{P}=\mathrm{C})$, the equipment driving production is the paver, and above the balance point, the equipment driving production is the batch plant. In the phase diagram representation (Figure 3), disequilibrium would be represented by the distance between system production line and the production of the paver (if $\mathrm{P}<\mathrm{C}$ ), or the batch plant (if $\mathrm{P}>\mathrm{C}$ ) (discussed later in Figure 24 . The complexity is the number of interactions of the system - when $\mathrm{P}=\mathrm{C}$, there are two interactions (plus the water truck interaction which is not shown in the phase diagram). However, as the number of interactions increase so do the number of possible phases, the degrees of freedom, and the available of deviations from the system equilibrium, meaning the complexity increases.

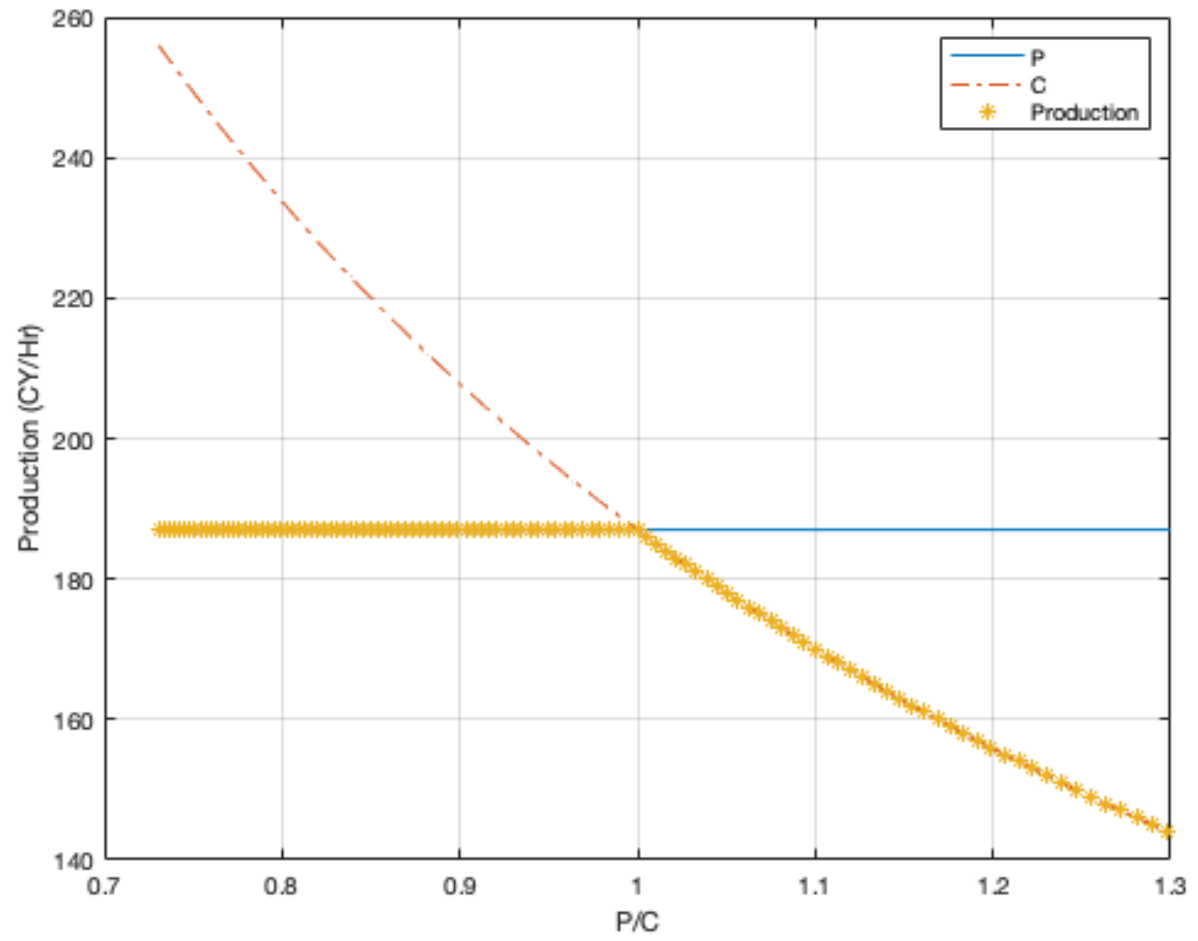

Figure 3: Phase diagram of paving construction example 


\subsection{Circular Representation}

A circle is an effective way of representing a cycle, especially if there are multiple interacting cycles involving multiple equipment. Consider the example of a gravel quarry operation involving a single excavator and a number of trucks $\left(n_{1}\right)$ working in tandem to excavate aggregate for a batch plant. Figure 4 shows the activity cycle diagram consisting of two cycles.

The truck cycle period is (time to load $\Delta_{0}$, travel to batch plant $t_{12}$, unload $\Delta_{1}$ and travel back $t_{11}$ ) denoted by $T_{1}$. Hence, the following can be asserted:

$$
T_{1}=\Delta_{0}+t_{11}+t_{12}+\Delta_{1}
$$

In this example, a single excavator requires $n_{0}$ cycles to load a single truck. The excavator cycle period (time to swing, cut, and dump in the truck) is denoted by $T_{0}$. Hence, the following can be asserted:

$$
n_{0} T_{0}=\Delta_{0}
$$

Then $n_{1}$ trucks are needed to balance the excavator production and ensure continuous operation.

$$
n_{1} \Delta_{0}=T_{1}
$$

Time to travel: $t_{11}$

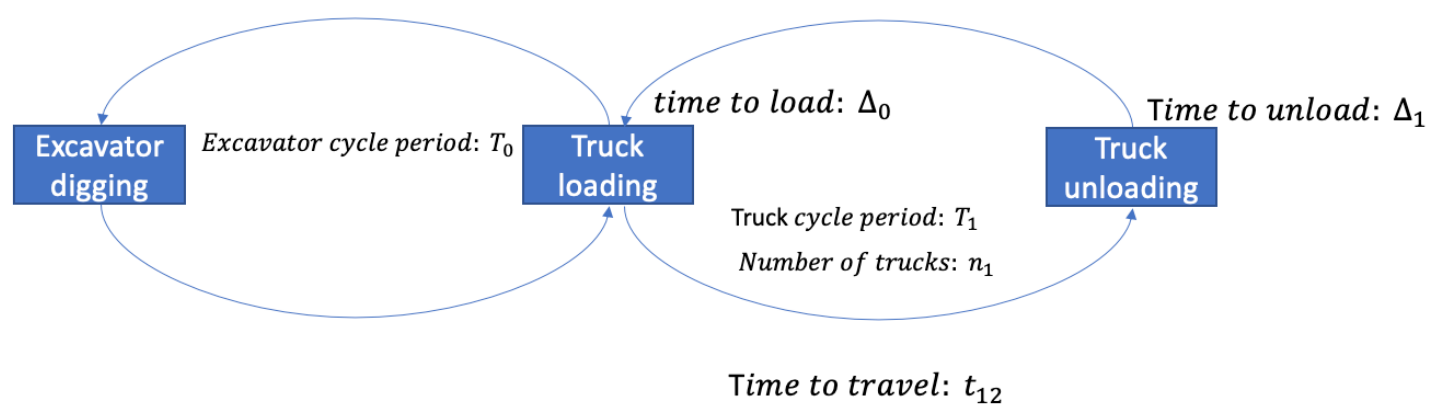

Figure 4: Example with an excavator and trucks. The excavator requires multiple cycles to load a truck.

Given the periodicity of cycles, the cycle time (or period, $T_{1}$ ) of the truck can be represented by the locus of a point around the circumference of a circle (Figure 5). This requires the theoretical construct of a radius of the cycle, $R_{1}$, such that:

$$
2 \pi R_{1}=T_{1}
$$


The circle represents the cycle time for the truck akin to a period. The partial hypocycloid inscribed in the circle represents the truck/excavator interactions. This hypocycloid is the locus of a fixed point on a circle with radius $R_{0}$ that is rolling inside the circle with radius $R_{1}$, where the radius $R_{0}$ corresponds to the cycle time of the excavator $T_{0}$ such that:

$$
2 \pi R_{0}=T_{0}
$$

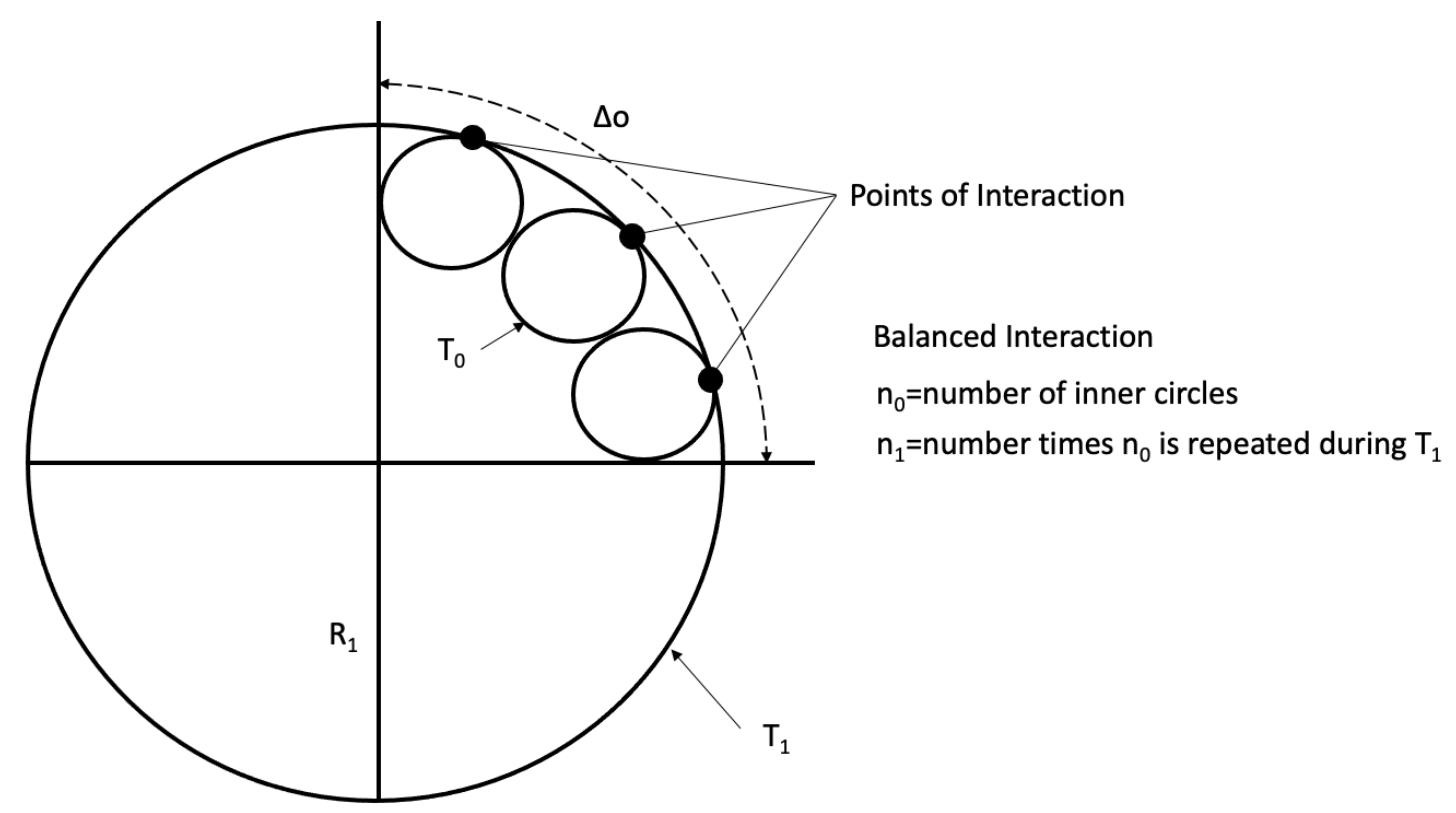

Figure 5: Balanced circle representation of excavator and truck interactions.

Figure 5 represents only the cycle time of one truck. The three interaction points in it shows that it takes three cycles of the excavator $\left(n_{0}=3\right)$ to completely load one truck. Once loaded, the truck continues on with the rest of its cycle traversing the circumference of the outer circle, until it returns back to interact with the excavator again - this time is denoted by: $t_{11}+t_{12}+\Delta_{1}$. For a perfectly balanced operation this is the same time that the excavator will use to load the other trucks. As there are $n_{1}$ trucks, it can therefore be asserted that:

$$
\left(n_{1}-1\right) \Delta_{0}=t_{11}+t_{12}+\Delta_{1}
$$

Figure 6 is replicated for each additional truck, with the excavator interactions seamlessly moving from one truck circle to the next as the excavator continues to load each truck without stopping. If the operation is perfectly balanced, with continuous operation, then each of the truck circles in Figure 6 can be superimposed into a single circle that represents all of the trucks and the excavator) and is illustrated in Figure 7. 


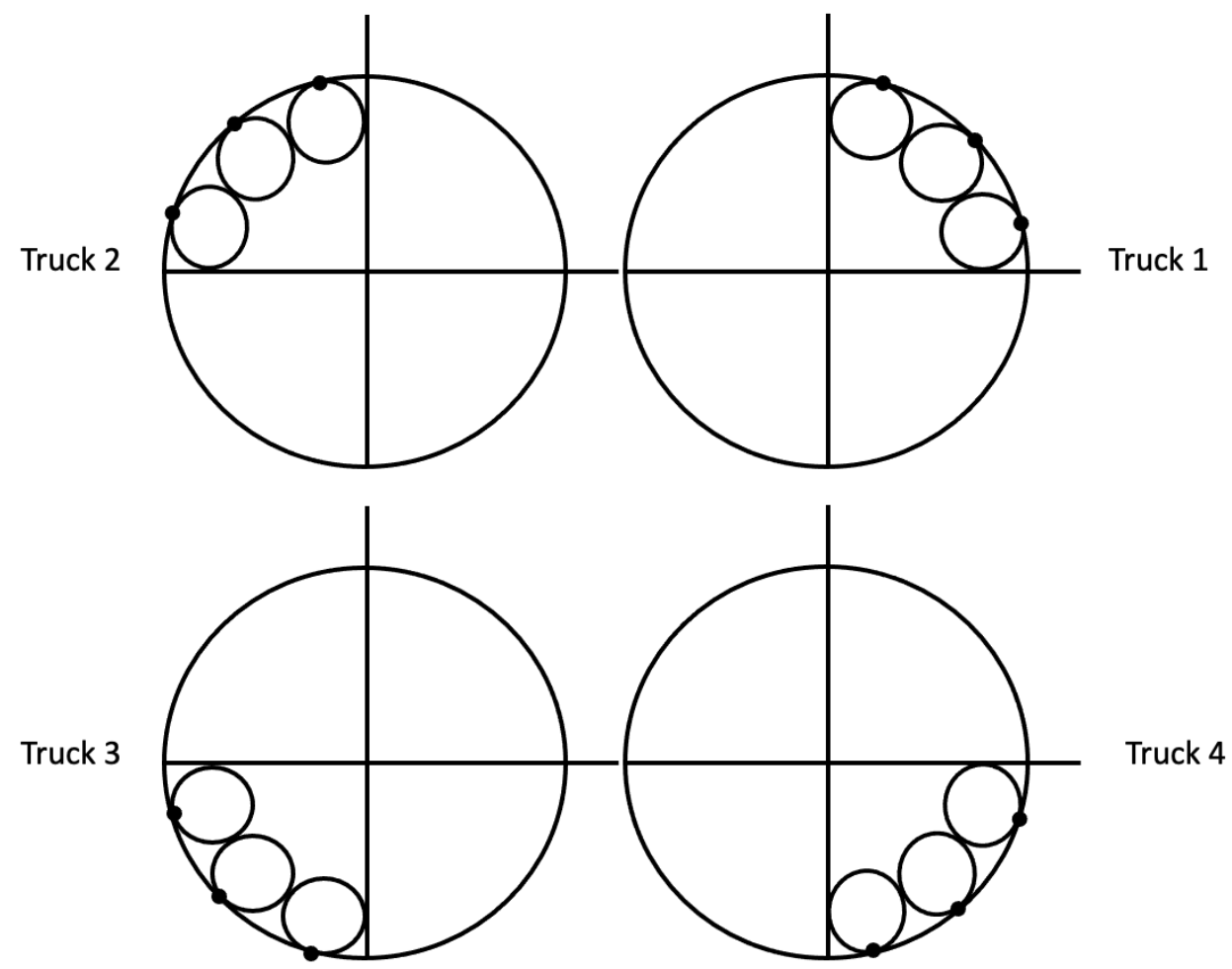

Figure 6: Excavator/truck interactions with multiple trucks.

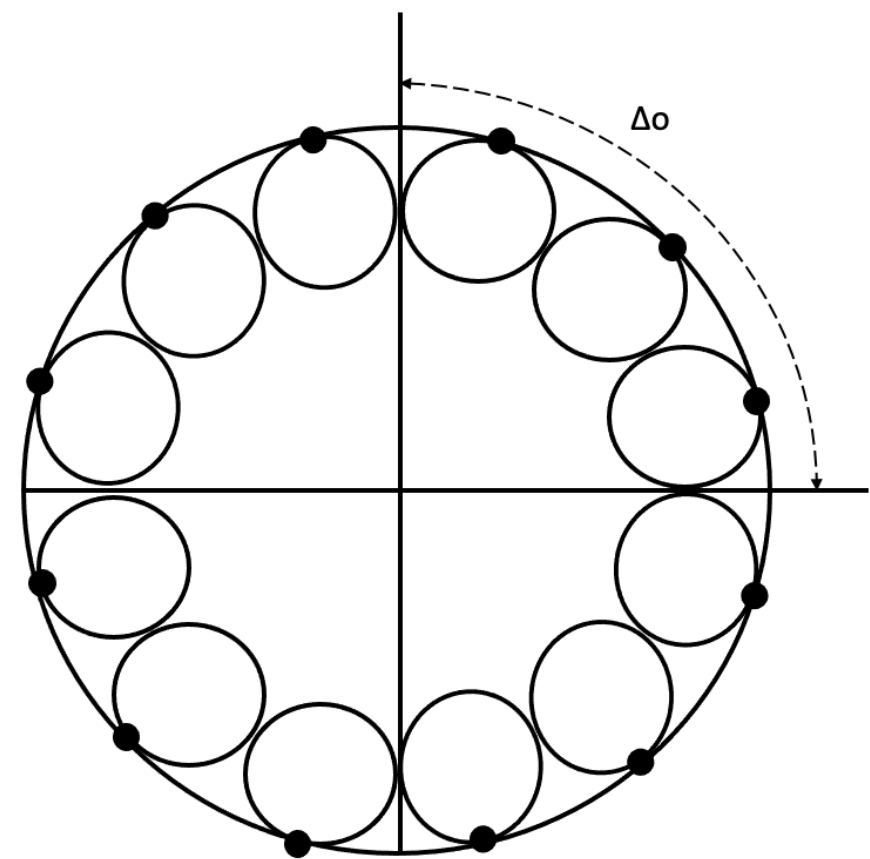

Balanced Interaction

Figure 7: Summation of truck/excavator interactions. The intersections between the circle and the hypocycloid are the interaction points between the trucks and the excavator. 
Hence, the single balanced interaction between the $n_{1}$ trucks with cycle time $T_{1}$ and one excavator with cycle time $T_{0}$, requiring $n_{0}$ cycles to load a single truck can be illustrated as a complete hypocycloid with a circle of radius $R_{0}$ rolling inside the circle of radius $R_{1}$. Combining equations 13 and 14, yielding:

$$
\begin{gathered}
n_{1} n_{0} T_{0}=T_{1} \\
n_{1}=\frac{T_{1}}{n_{0} T_{0}}
\end{gathered}
$$

Hence, for a system in equilibrium, the product of the number of trucks and excavator cycles (or number of excavators) is the ratio of the cycle times. $n_{0}$ and $n_{1}$ must be integers, therefore the constraints on loading times and production rates are less flexible. When additional interacting equipment cycles are added, this formulation can be recursively extended as illustrated in Figure 8. The system equations can be expressed generally as follows:

$$
n^{\prime}{ }_{1} T^{\prime}{ }_{1}=\Delta_{1}
$$

Where $n_{1}^{\prime}$ is the number of trucks to keep the batch plant busy, $T_{1}^{\prime}$ is the cycle time of the trucks to keep the batch plant busy, and $\Delta_{1}$ is the truck unloading time.

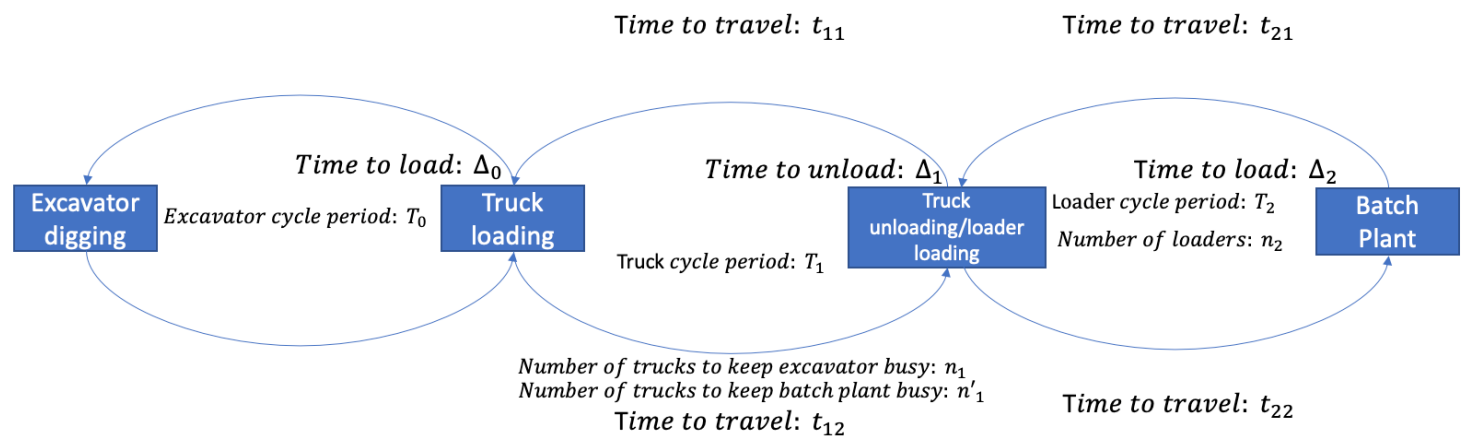

Figure 8: Adding a loader that takes the material from the truck to a batch plant.

The total cycle time of the loader, $T_{2}$, is a function of the unloading time of the truck $\Delta_{1}$, the travel times of the loader $\left(t_{21}\right.$ and $\left.t_{22}\right)$.

$$
\Delta_{1}+t_{21}+t_{22}+\Delta_{2}=T_{2}
$$

The number of trucks needed at $\Delta_{1}$ is:

$$
\frac{T_{2}}{\Delta_{1}}=n_{2}
$$


The loader time to transport and unload gives:

$$
\left(n_{2}-1\right) \Delta_{1}=t_{21}+t_{22}+\Delta_{2}
$$

Substituting equation 23 into equation 21 gives:

$$
\left(n_{2}-1\right) \Delta_{1}=T_{2}-\Delta_{1}
$$

Distributing $\Delta_{1}$ out and using equation 20 to substitute for $\Delta_{1}$

$$
\begin{gathered}
n_{2} n^{\prime}{ }_{1} T^{\prime}{ }_{1}=T_{2} \\
\text { If } n_{1}^{\prime}=n_{1} \text { and }{T^{\prime}}_{1}=T_{1}
\end{gathered}
$$

then, the system is balanced. This provides the product of the number of loaders and trucks with the truck cycle time is equal to the cycle time of the loader:

$$
n_{2} n_{1} T_{1}=T_{2}
$$

Substituting in for the excavator cycle time $T_{0}$ and the number of cycles of the excavator, $n_{0}$ :

$$
\begin{gathered}
n_{2} n_{1}\left(n_{1} n_{0} T_{0}\right)=T_{2} \\
n_{0} n_{1}{ }^{2} n_{2} T_{0}=T_{2}
\end{gathered}
$$

Therefore, the cycle time $T_{\mathrm{m}}$ of any equipment in the construction cycle can be related to the quantity of other equipment and the initial equipment cycle time $T_{0}$ by:

$$
\begin{gathered}
T_{m}=n_{0} n_{1}{ }^{2} n_{2}{ }^{2} n_{3}{ }^{2} \ldots n_{m-1}{ }^{2} n_{m} T_{0} \\
T_{m}=\prod_{k=0}^{m} n_{k} \prod_{p=1}^{m-1} n_{p} T_{0}
\end{gathered}
$$

Equation 31 allows us to mathematically express the balance conditions for an operation with $m$ interaction points.

The expansion of systems can occur in multiple directions. Systems can be linear (as in Figure 8) or can be in branch configurations (Figure 9). Additional equipment and additional interactions increase the potential for disequilibrium (more intersections must align, reducing the solution space). As the number of interactions increases, the layers of the circle diagram increase. Figure 10 shows the concept of nested circle diagrams. Each level (nested circle) represents another equipment component. The radius of each circle is determined by equation 15 . 


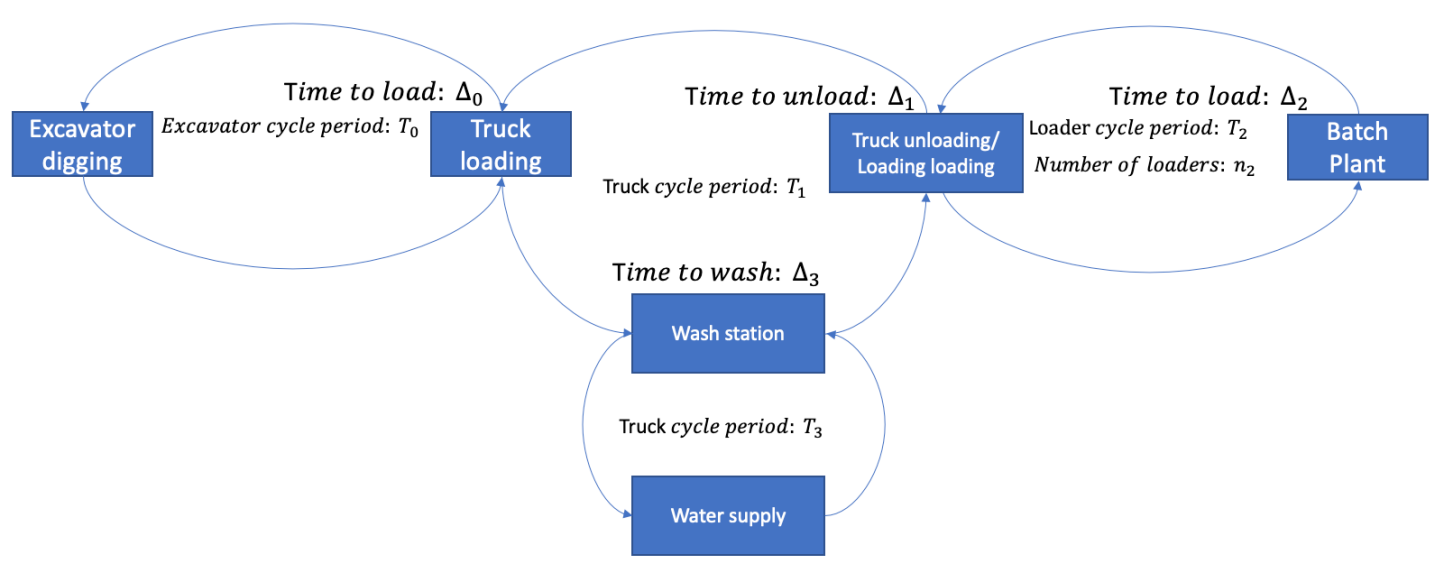

Figure 9: Branch configuration of a system.

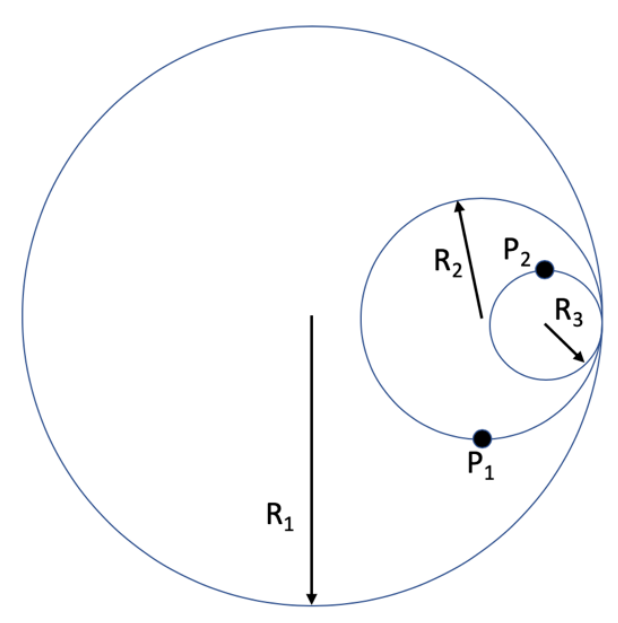

Figure 10: Nested circle diagram. Each layer represents a component interaction.

In a circular representation of a system, disequilibrium would appear as an incongruence of the inner hypocycloid and outer circle. The incongruence can be expressed as a phase angle difference or as a time delay. Figure 11 illustrates the truck excavator example with delay $\varepsilon$ in the system. 


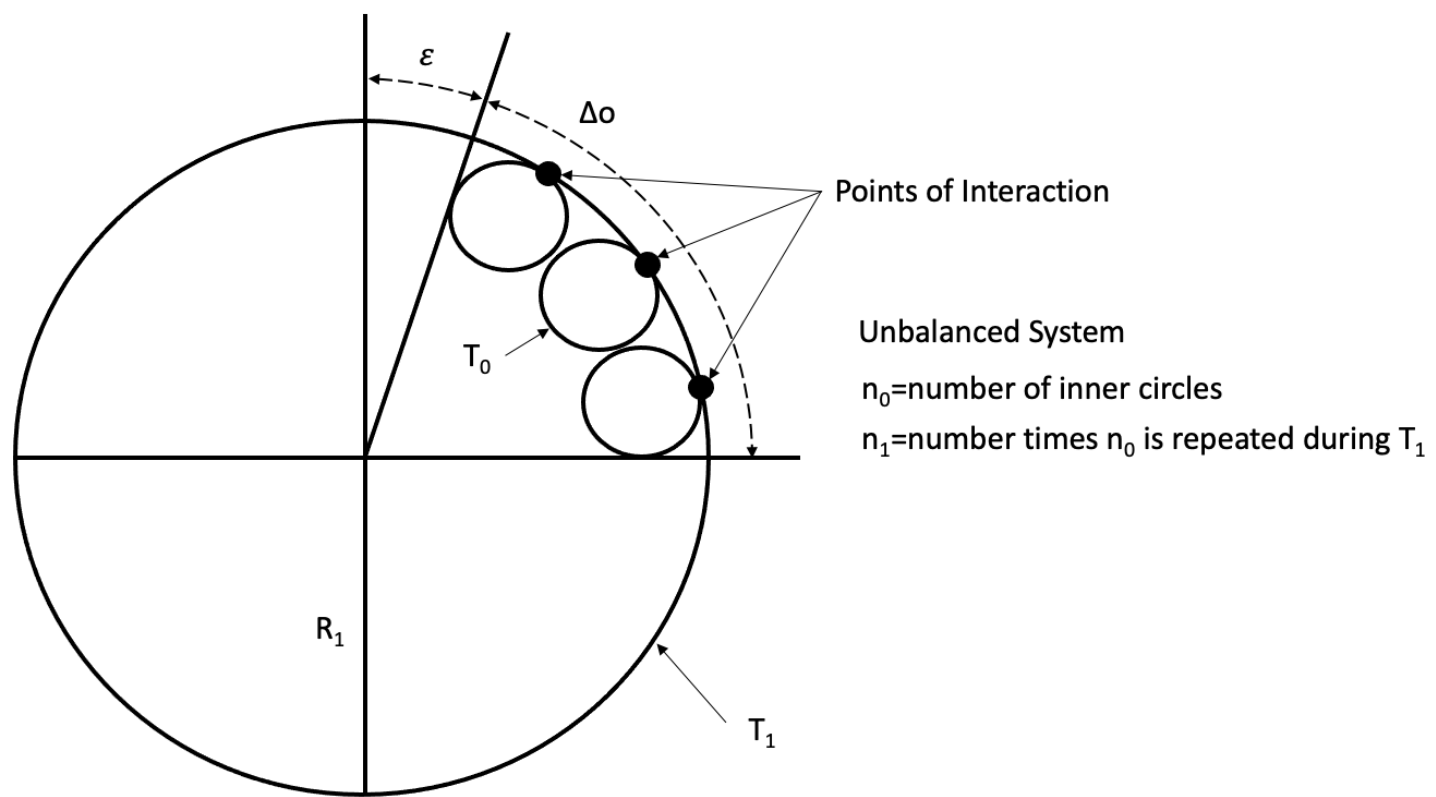

Figure 11: Unbalanced circle representation of truck and excavator interactions.

The equations for an unbalanced system must account for $\varepsilon$. Equation 14 is modified to:

$$
n_{1}\left(\Delta_{0}+\varepsilon\right)=T_{1}
$$

Or,

$$
\Delta_{0}=\frac{T_{1}-n_{1} \varepsilon}{n_{1}}
$$

And from equation 13:

$$
n_{0} T_{0}=\Delta_{0}
$$

Becomes:

$$
n_{1} n_{0} T_{0}=T_{1}-n_{1} \varepsilon
$$

If $T_{1}$ is set at 1 (time period) as a reference value, then:

$$
T_{0}=\frac{\left(1-n_{1} \varepsilon\right)}{n_{1} n_{0}}
$$

The phase diagram and circular diagram are not both ideal indicators for all systems. For systems that need multiple interactions with the same equipment types (for example, the excavator taking multiple cycles to load a truck), the circular representation provides a better visual indication of 
multiple cycle interactions. However, for a single interaction (one cycle for each equipment) the phase diagram provides a more useful depiction of the interactions.

\subsection{Waveform Representation}

Using the example of an excavator and truck in a digging, loading, hauling system, (Figure 12) the excavator and truck each can be represented by a waveform and their interactions and disequilibrium visually seen.

Time to travel: $t_{11}$

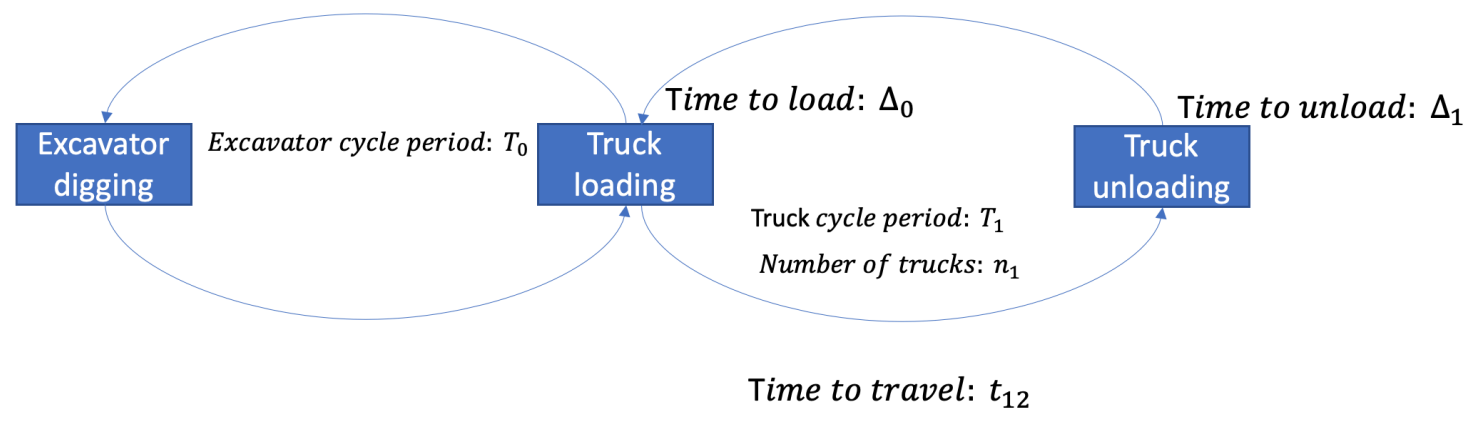

Figure 12: Example with an excavator and trucks. The excavator requires multiple cycles to load

a truck.

Starting from the number of trucks $n_{1}$, the truck cycle period $T_{1}$ is the cycle time results in the following parameters of the waveform (note, normally signals are referred to in $\operatorname{Hertz}(\mathrm{Hz})$ or cycles per second. However, in this dissertation, the frequency will be represented in cycles per unit time or CPT for notation purposes as the frequency in Hertz would be very small and cumbersome to work with and tiresome to read):

$$
f_{1}=\frac{1}{T_{1}}
$$

The frequency is a function of the cycle period. As the truck is the reference component in this example, the frequency is assigned as 1 CPT.

$$
f_{1}=1 \mathrm{CPT}
$$

- $n_{1}=4$, the number of trucks is 4 for this example.

- $n_{0}=3$, as it takes 3 excavator cycles to fill the truck.

- $\varepsilon$ is the delay when either component (truck or excavator) needs to wait for the other. Non-zero values of $\varepsilon$ indicate an unbalanced system (disequilibrium). 
Using these parameters, the signal for the truck is given by:

$$
\begin{gathered}
y_{1}=\sin \left(f_{1} *(t-\varepsilon)\right) \\
y_{1}=\sin (1 *(t-\varepsilon))
\end{gathered}
$$

Plotting for several cycles produces the plot in Figure 13 showing the truck waveform. For these plots there is no $y$-labeling as the $y$-value is a construct derived from equation 15. Key takeaway from these plots is the relative frequency to each component.

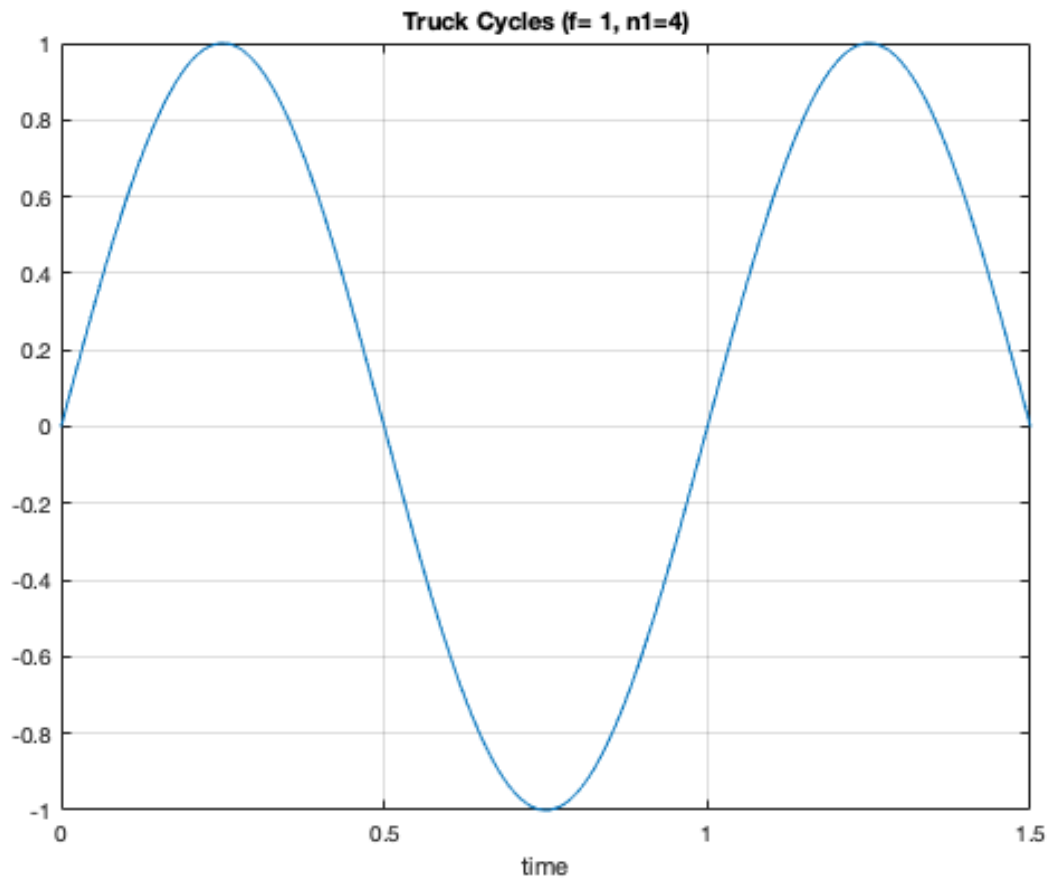

Figure 13: Waveform of a single truck.

Similarly, for the excavator, the frequency is $n_{0} \times n_{1}$. This is due to the 3 excavator cycles needed to load a truck, and then the truck cycle period being 4 times the truck loading time.

$$
\begin{gathered}
f_{2}=n_{0} * n_{1}=3 * 4=12 \\
\mathrm{y}_{2}=\sin (12 *(t-\varepsilon))
\end{gathered}
$$

This results in the plot in Figure 14: Waveform of excavator, showing the excavator frequency is 12 CPT. 


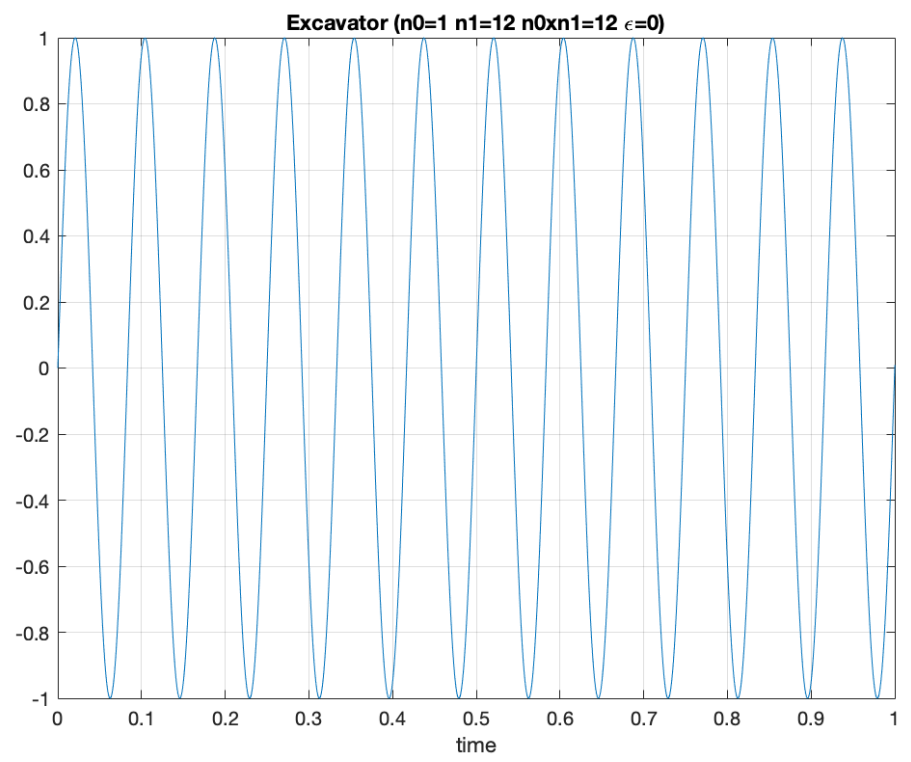

Figure 14: Waveform of excavator

Of interest, is the combination of these waveforms to determine the system waveform interactions. Figure 15 shows the sum of the truck and excavator waveforms.

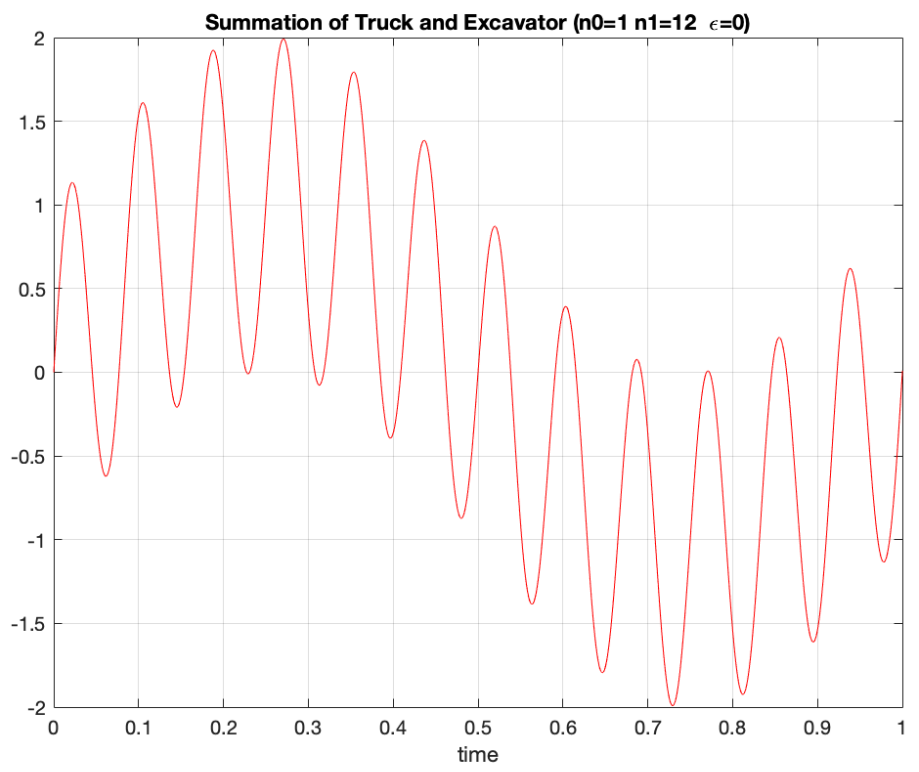

Figure 15: Sum of truck and excavator waveform.

To analyze the combined waveform, a Fast Fourier Transform (FFT) is used (equation 43) to decompose the combined signal into its component frequencies. The FFT is a computationally efficient algorithm to calculate the Fourier transform of a discrete time signal (note, while the 
actual signals in these examples are continuous, they can be represented by discrete time simulations (such as in the plots in this research). The plots in this dissertation look at a small segment of the signal, usually 1 to 1.5 cycles. Therefore, in order to improve the frequency resolution, the signals are zero padded to increase the signal length up to next "power of 2" number of samples $\left(\mathrm{N}^{2}\right)$. Additionally, the sampling is greater than twice the Nyquist rate to ensure aliasing is not occurring, which would provide erroneous results in the frequency domain plots, and the number of samples $(\mathrm{N})$ are sufficiently large as to be an integer number of periods to avoid time leakage.

The FFT shows the two frequencies. Using the method of a FFT is not bidirectional. First, projects or emergency responses do not have signals to record. Second, while the FFT can be used to decompose signals into component frequencies, this does not necessarily indicate the number of interactions. However, this method is intended to show that interacting components can generate signals and the FFT can show how the inherent structure of a project/event can be visualized as the number of components or as delays in components vary.

$$
X(k) \sum_{n=0}^{N-1} X(n) e^{-j 2 \pi / N}, \quad \text { for } 0 \leq k \leq N-1
$$

Where, $X(k)$ is the $k^{\text {th }}$ harmonic and $X(n)$ is the $n^{\text {th }}$ input sample.

Plotting the FFT of the summation of the truck and excavator signals (Figure 16) indicates the presents of two frequencies: 1 and 12 CPT, matching the input frequencies for this example.

The next question is: what happens as the number of system components grows? If there is a third component (a loader for example that the truck dumps into), how does the additional component impact the system waveform. As expected, the addition of another component's waveform shows up on the FFT as another frequency present (Figure 17). This pattern continues to scale as additional components are added. Figure 18 shows an example with 5 components. As the number of components $N$ goes to infinite, the number of frequencies present will also go infinite, provided they are unique frequencies. Repeated frequencies will show up as stronger signal strength in the FFT magnitude plot. 

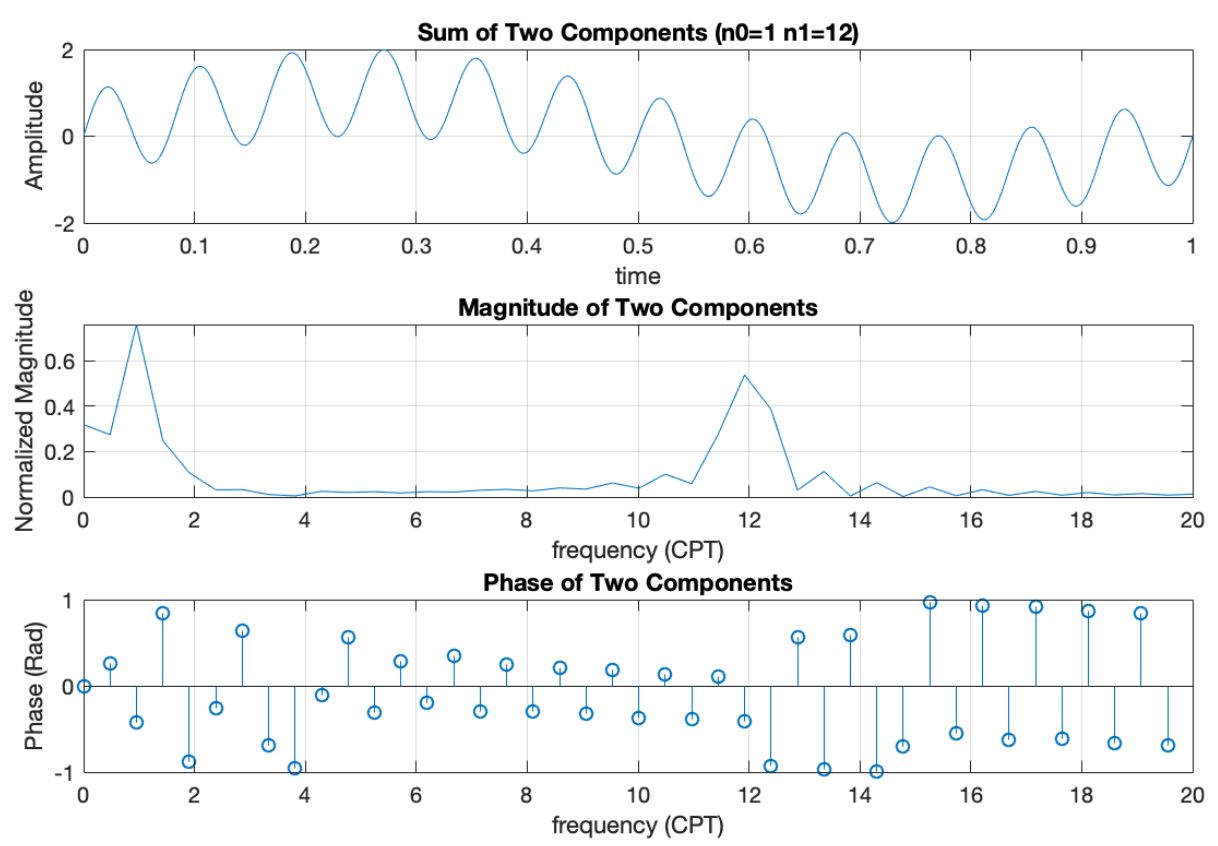

Figure 16: The sum of two components and the FFT magnitude and phase of the sum.
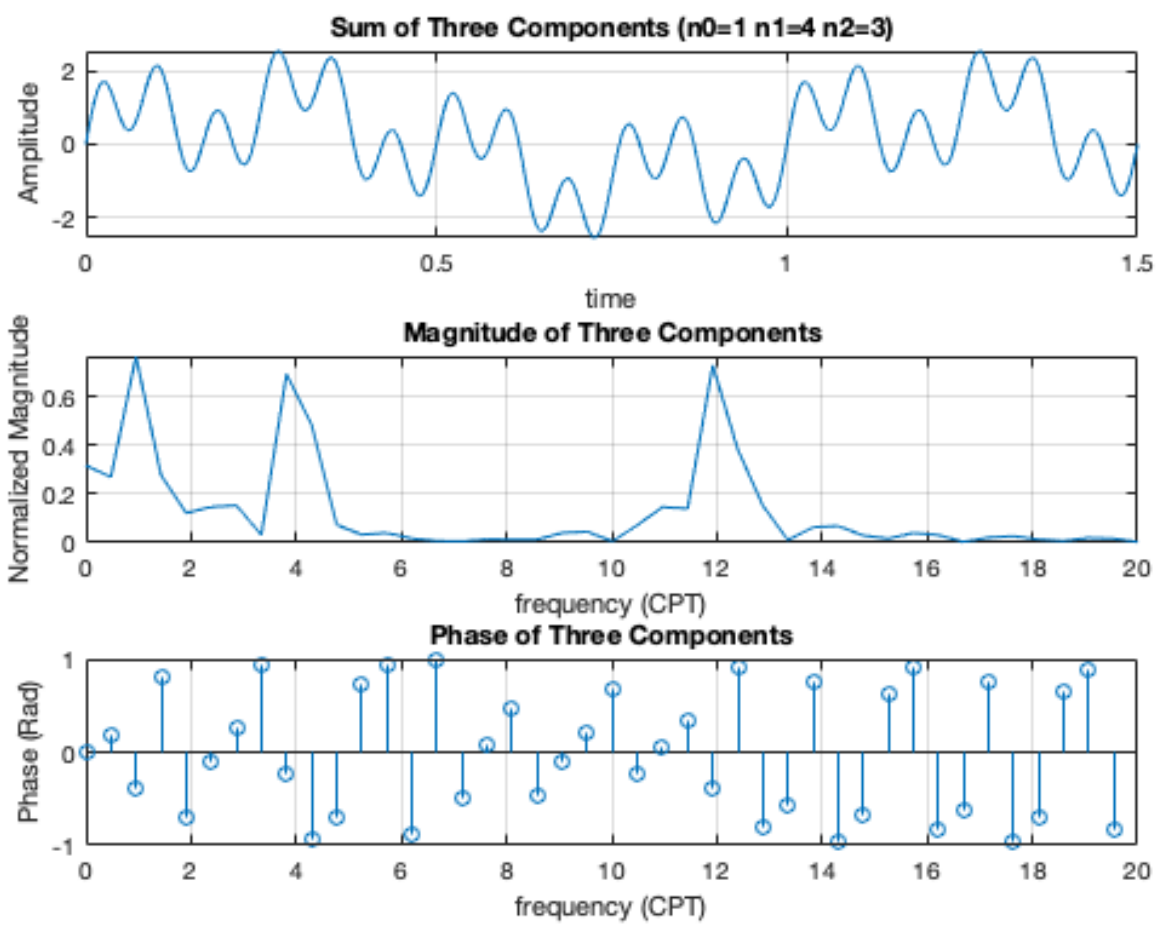

Figure 17: Summation of three signals. The three frequencies are present in the second plot. 


\section{Sum of Varying Frequency}
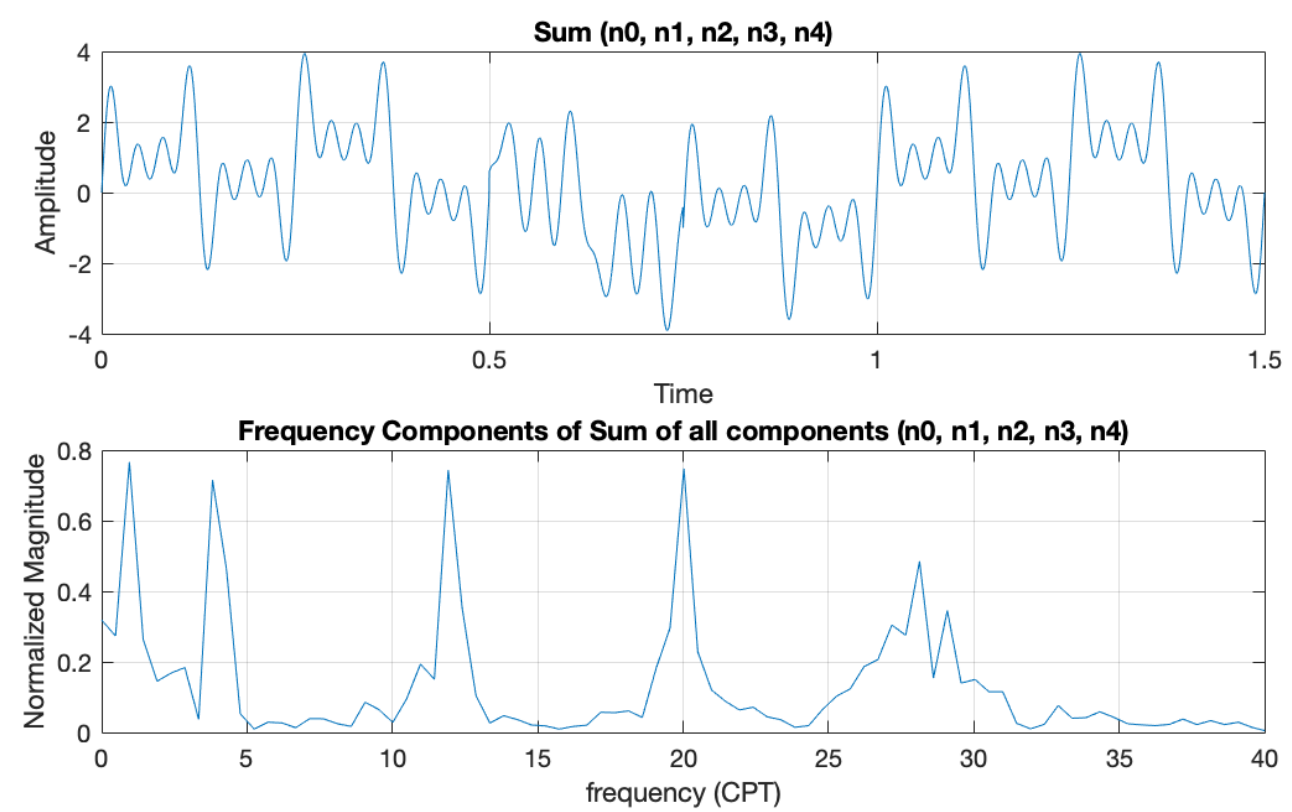

Phase Angle of sum

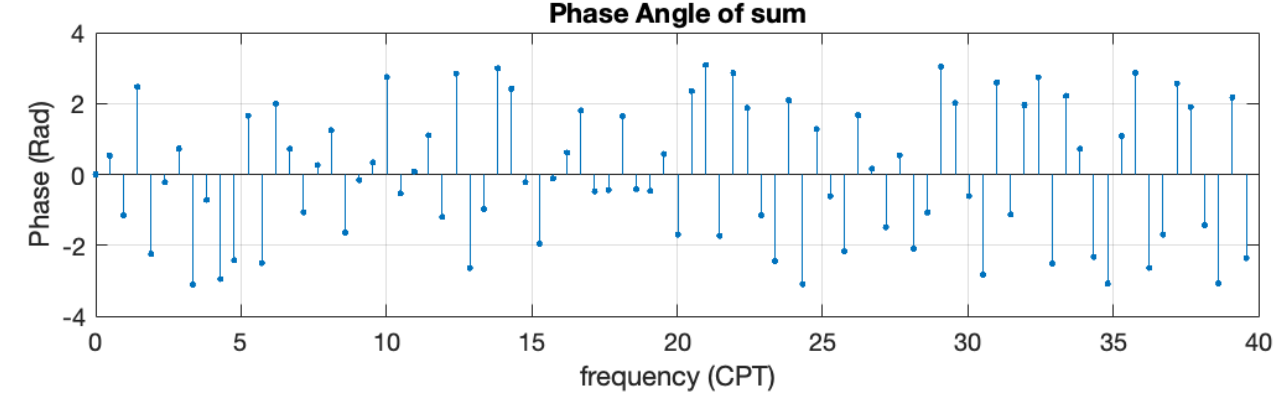

Figure 18: Summation of 4 signals, the FFT magnitude, and phase

Additional comparisons of frequencies are included in $6 \mathrm{~A}$

\subsubsection{Infinite Components}

As the number of components or interactions increases to infinity, the waveform continues to evolve. Figure 19 shows what happens for very large numbers of components at random frequencies. As the number of frequencies increases, the overall of the sum will continue to increase. Figure 20 shows the sum of large numbers of frequency waveforms but with a linear increase in frequency rather than random frequencies. The implication is that emergence is not just a function of interactions but the order or topology of those interactions impacts the results. Once conjecture is that the differing results between the random and sequential frequency summations are an artifact of sampling. 

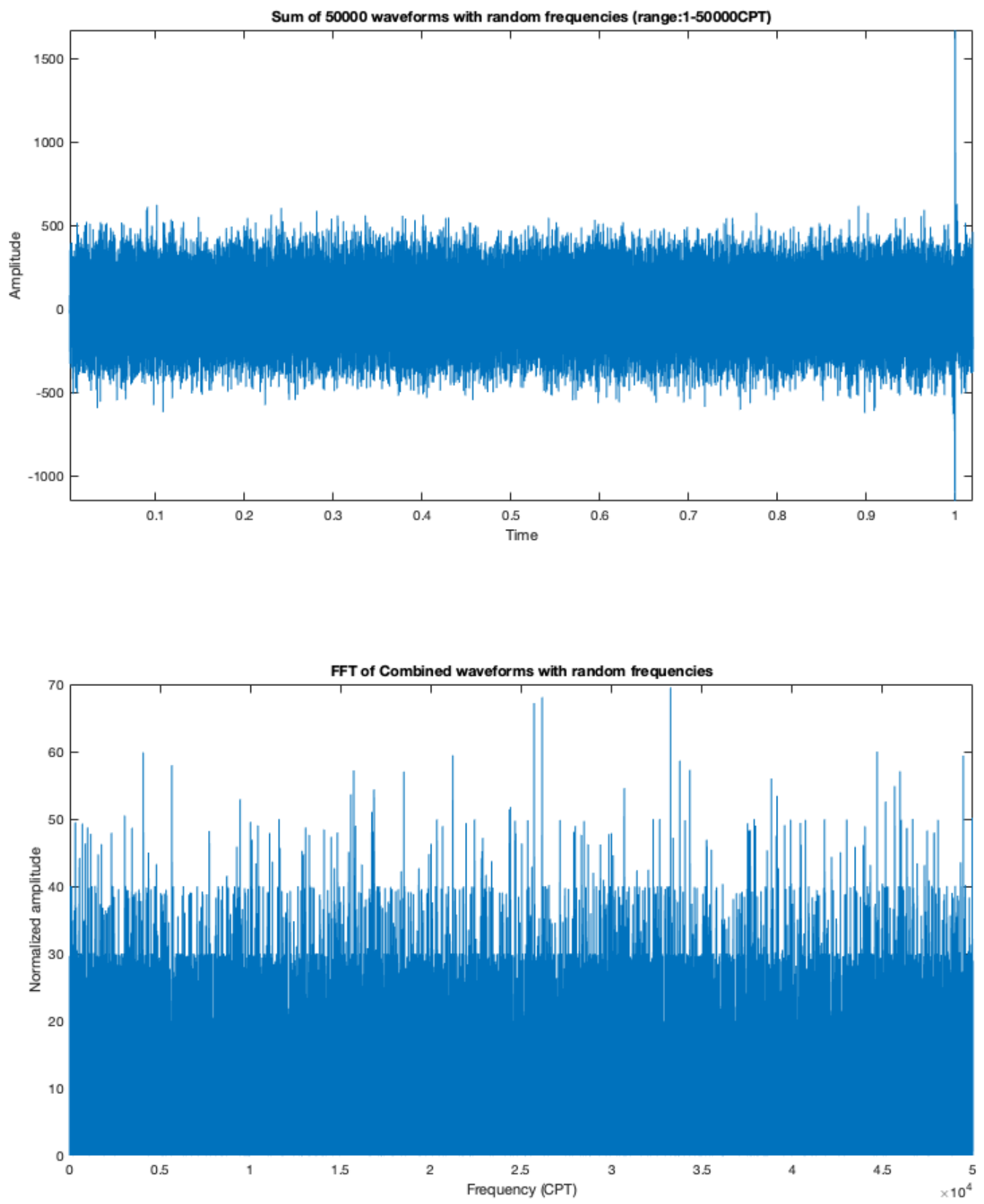

Figure 19: Sum of very large numbers of frequencies ( $n=50,000$ random frequencies) and the FFT normalized magnitude showing the random frequencies used. 

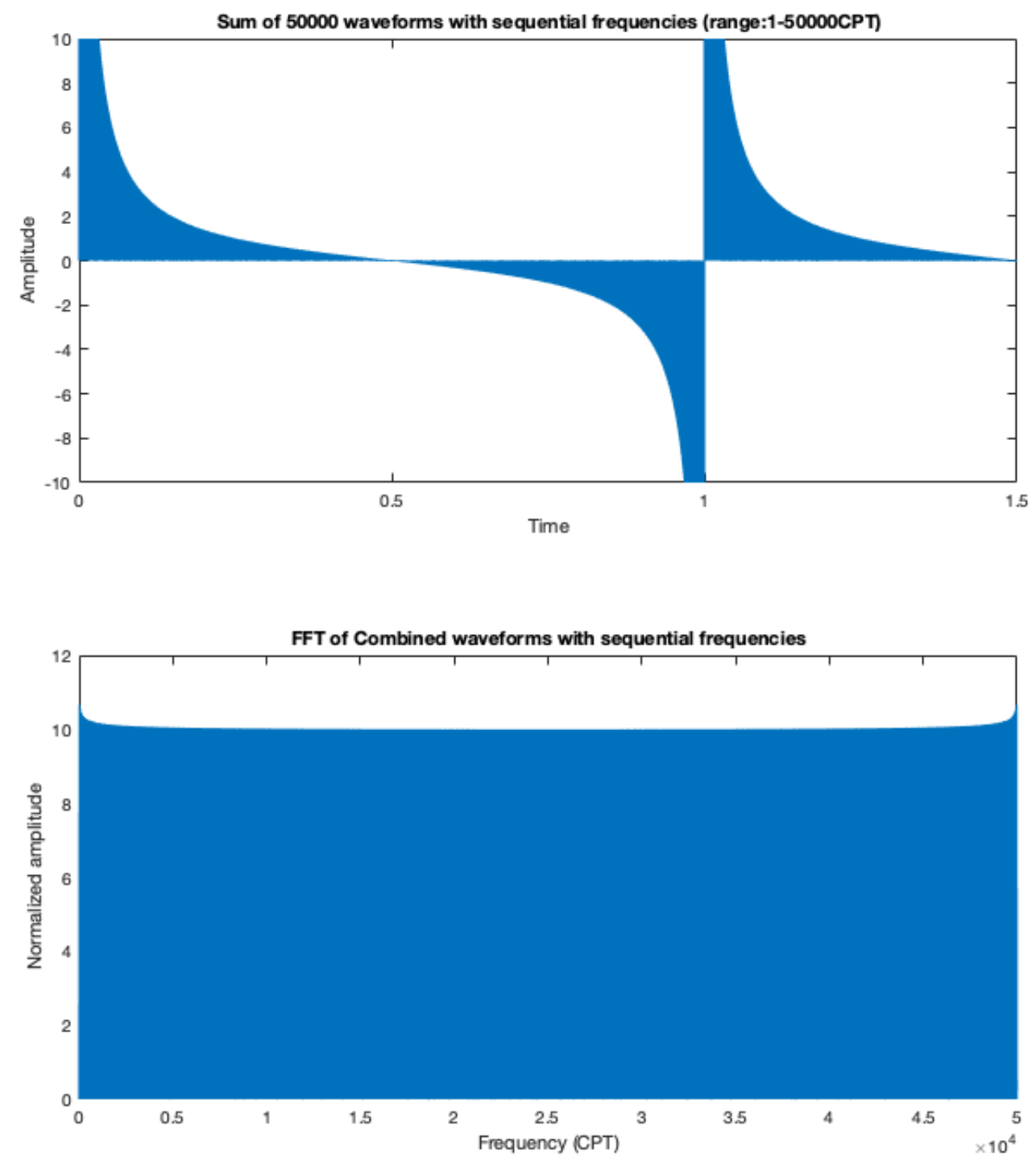

Figure 20: Sum for very large number of frequencies ( $n=50,000$, linearly increasing) and the FFT normalized magnitude.

\subsubsection{Unbalanced Systems}

These examples thus far have assumed the system is in equilibrium (balanced). However, if the system has delay (disequilibrium) the waveform is impacted. The plots in Figure 21 show the excavator waveform "waiting" followed by its next three cycles. This makes a noticeable impact when the truck and excavator waveforms are added. The FFT is also computed and shows two frequencies present (1 CPT and 4 CPT) as before as well as a small indication of a signal at 7.6 CPT. This additional frequency is a result of the delay in the system skewing the "true" frequency of the excavator waveform. 

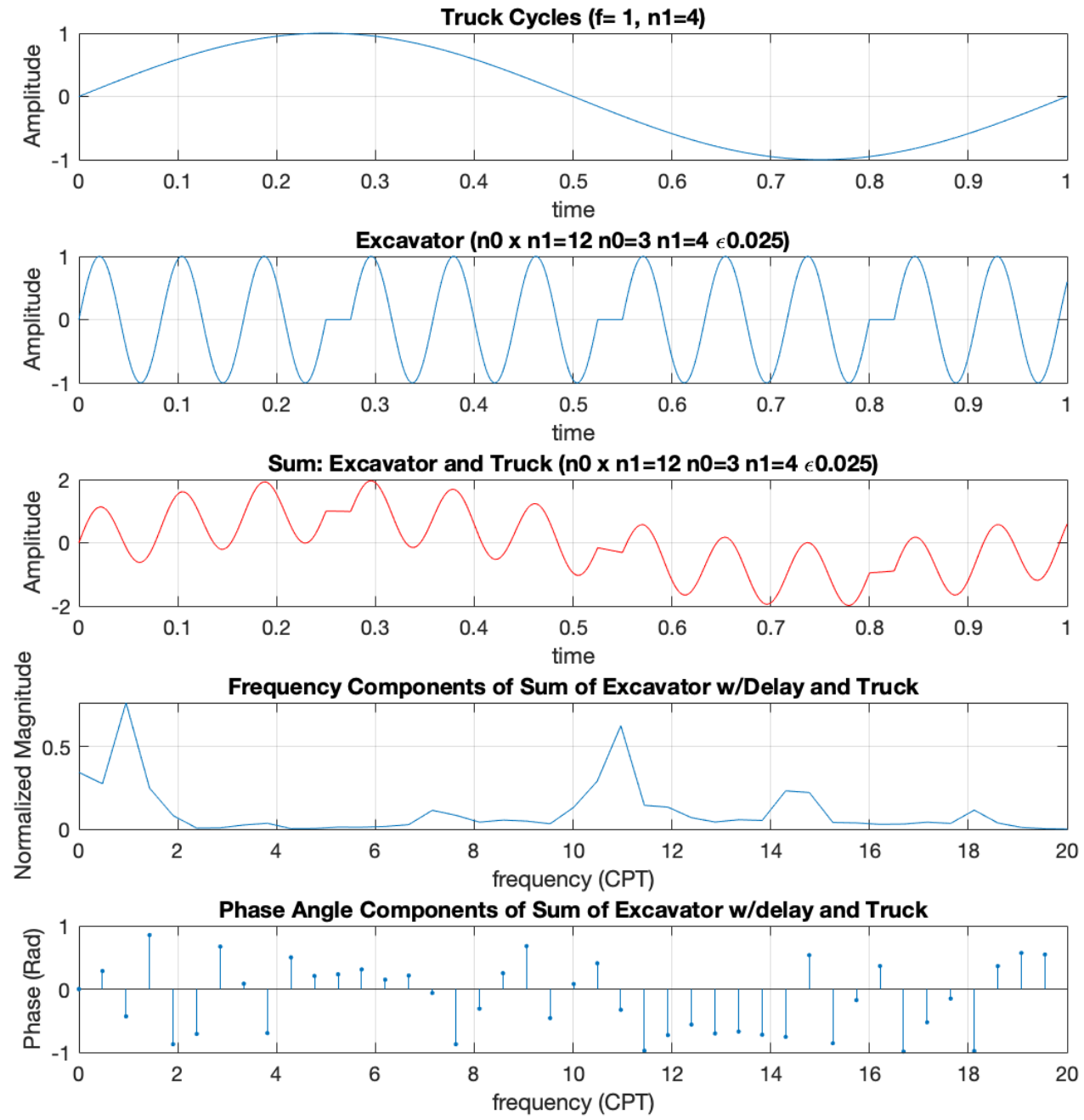

Figure 21: Two component signals with the excavator signal delayed by 0.025 .

As the delay increases, the results of the FFT of the sum of the truck and excavator waveforms are similar (Figure 22), with additional harmonic frequencies present. This result means that as the delay in the system increases, the disequilibrium increases. 

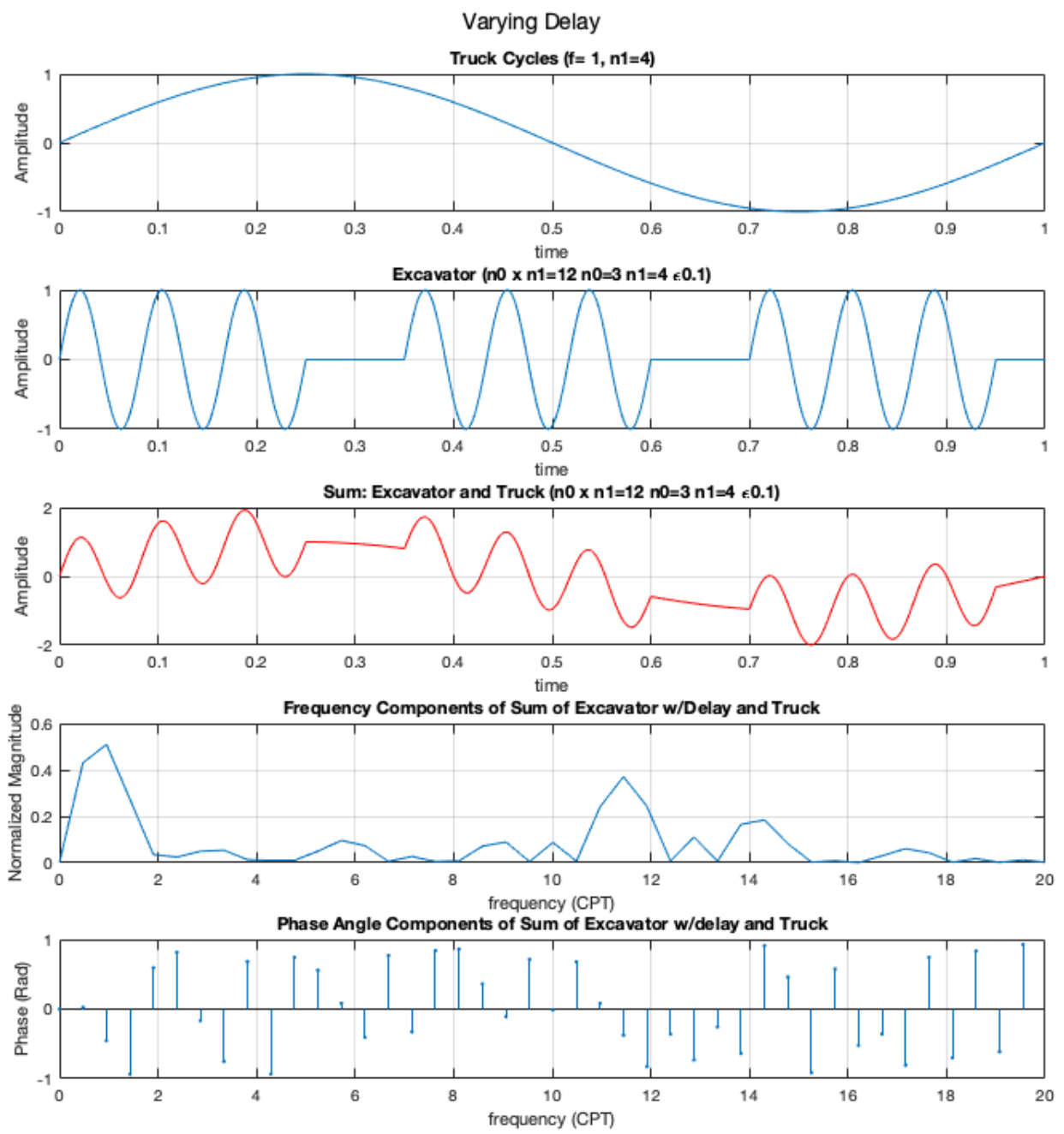

Figure 22: As delay increases, the FFT indicates additional frequencies present as harmonics.

Next, combining both 5 frequencies (components), each at a different delay, produces Figure 23 which shows the summation waveform. The FFT of the waveform, however, shows the 5 frequencies present but also the harmonics resulting from the delays in the component waveforms. The result shows the FFT can be used to identify the frequency components of interacting components can help visualize the underlying system and the impact of delay (disequilibrium) in a system. 

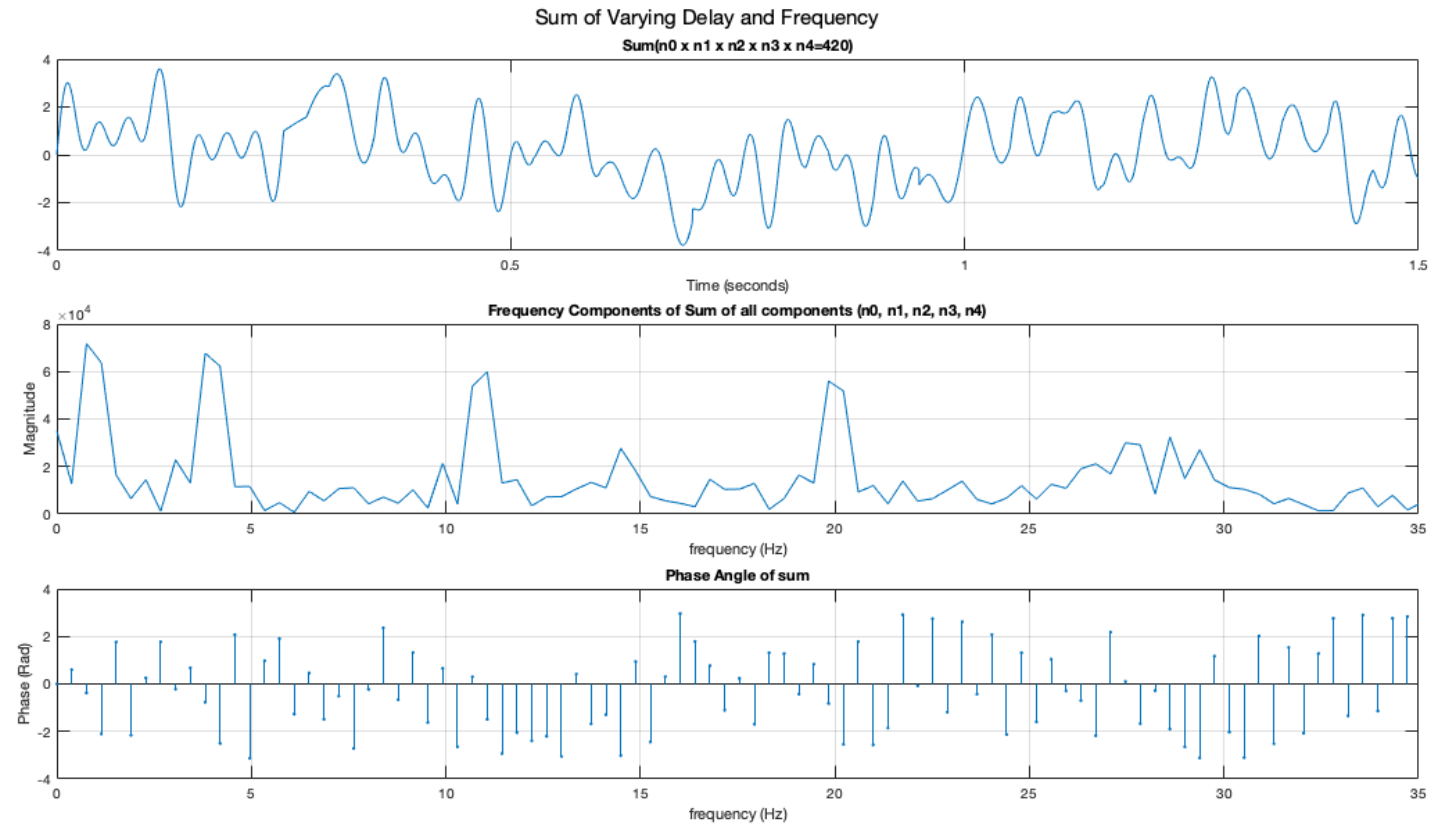

Figure 23: Results of varying both frequency and delay.

\subsection{Metric of Complexity}

There are four primary takeaways from the methods discussed above:

1. From the paver example in Figure 2, the system is not in equilibrium, estimating the net production rate requires additional variables $\left(n_{1}\right)$ that can be eliminated when the system is in equilibrium. Thus, a system in equilibrium is more easily reducible than a system in disequilibrium and requires management of fewer information parameters. The phase diagram (Figure 3) helps identify the conditions under which equilibrium can be maintained (when $\mathrm{P}=\mathrm{C}$ in the Figure 3 example) while the phase diagram in Figure 24 shows a system that is in disequilibrium because $n_{0} / n_{1}$ is not equal to 1 , and therefore, the operating point is not at the balance point - causing the paver to be idle. The disequilibrium makes these cases irreducible and according to the definition by Crutchfield and Young (1989), higher complexity. 


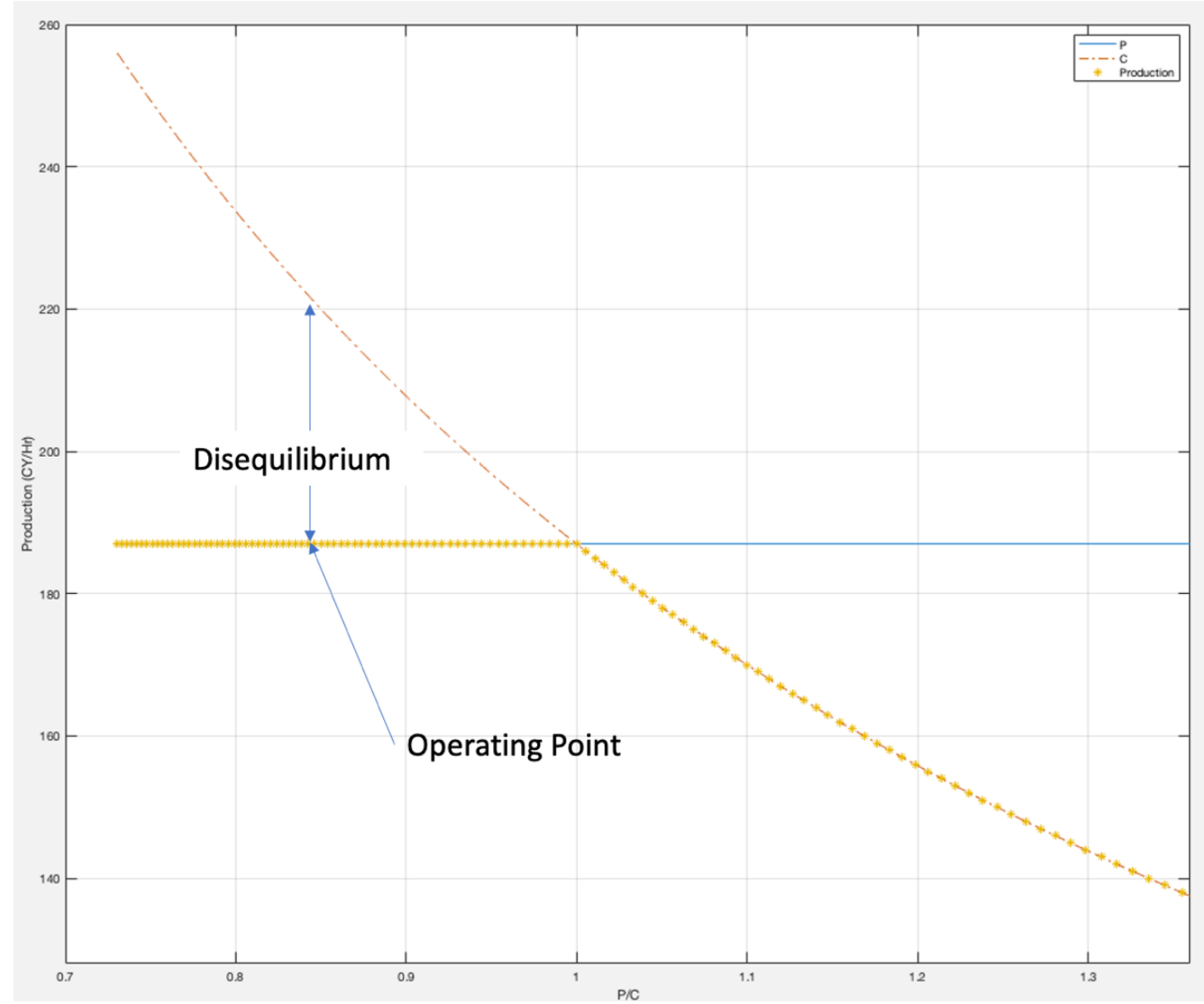

Figure 24: Phase diagram showing disequilibrium at the operating point.

2. The circular diagrams indicate that as the number of interactions increase so do the number of balance points, in a multiplicative fashion, adding to the number of potential deviations from equilibrium conditions, and combinations thereof. This leads to a very large number of possible irreducibilities in the system.

3. Realistically, systems tend to equilibrium conditions, but rarely function in perfect equilibrium. In addition, the extent of movement away from an equilibrium condition can also be difficult to measure and translate into an easily applicable metric (e.g., consider developing a distribution of delay times). However, reducing the number of interactions can reduce the number of ways in which a system can deviate from equilibrium, and reduce the number of possible points that need to be managed. Managing the level of disequilibrium at each interaction, or minimizing the deviation at each balance point, can help reduce the complexity of the project. In other words, an operation design with fewer interactions and operating close to the balance point conditions will have a lower complexity and will be likely to have better project objective outcomes.

4. Using the Fast Fourier Transform of the combined waveforms will identify the component waveform frequencies helping to reveal the underlying structure of the system. The disequilibrium is identified by the appearance of harmonic frequencies.

Hence, this dissertation posits that complexity is a function of the number of interactions because these are points where disequilibrium can exist. The disequilibrium is the indication of an 
information flow failure, but the complexity is the number of interactions. To reduce complexity, the objective is to reduce the number of driving interactions and manage the project to ensure that each interaction is as close to equilibrium conditions as possible.

Therefore, the proposed metric of complexity is the number of defining interactions in the system, given a management principle. As the number of interactions increases, so do the degrees of freedom for the system. As degrees of freedom increase, each interaction has the possibility of being in disequilibrium from the expected or normal value. Disequilibrium is demonstrated in the components of analysis: block diagram, waveforms analysis, phase diagram, circular diagram, and equation 31. These are all representations of cycles and show interaction points and disequilibrium between components.

\subsection{Organizational Complexity}

So far, the discussion has used operational examples to illustrate how the number of interactions is a metric of complexity. However, the same metric can be very usefully applied to organizational systems as well. In the case of construction operations, the continuous operation principle is used as a symmetry to assess system reducibility by establishing balance points as equilibrium conditions. Organizationally, similar principles that reduce lag times and improve communication cycle times can be used to define a symmetry that reflects the context at hand. For example, consider the following hypothetical scenario depicted in Figure 25: The contractor (hired by an Owner) has 4 sub-contractors. Each sub-contractor can be in a fiscal state of "under budget", "on budget", or "over budget". In this case, the symmetry is the budget ("on budget"). There are 5 interactions (between the contractor and each sub-contractor, and the owner and the contractor). The measure of complexity is a function of the number of interactions (in this case 5). There is disequilibrium in 4 of the 5 interactions.

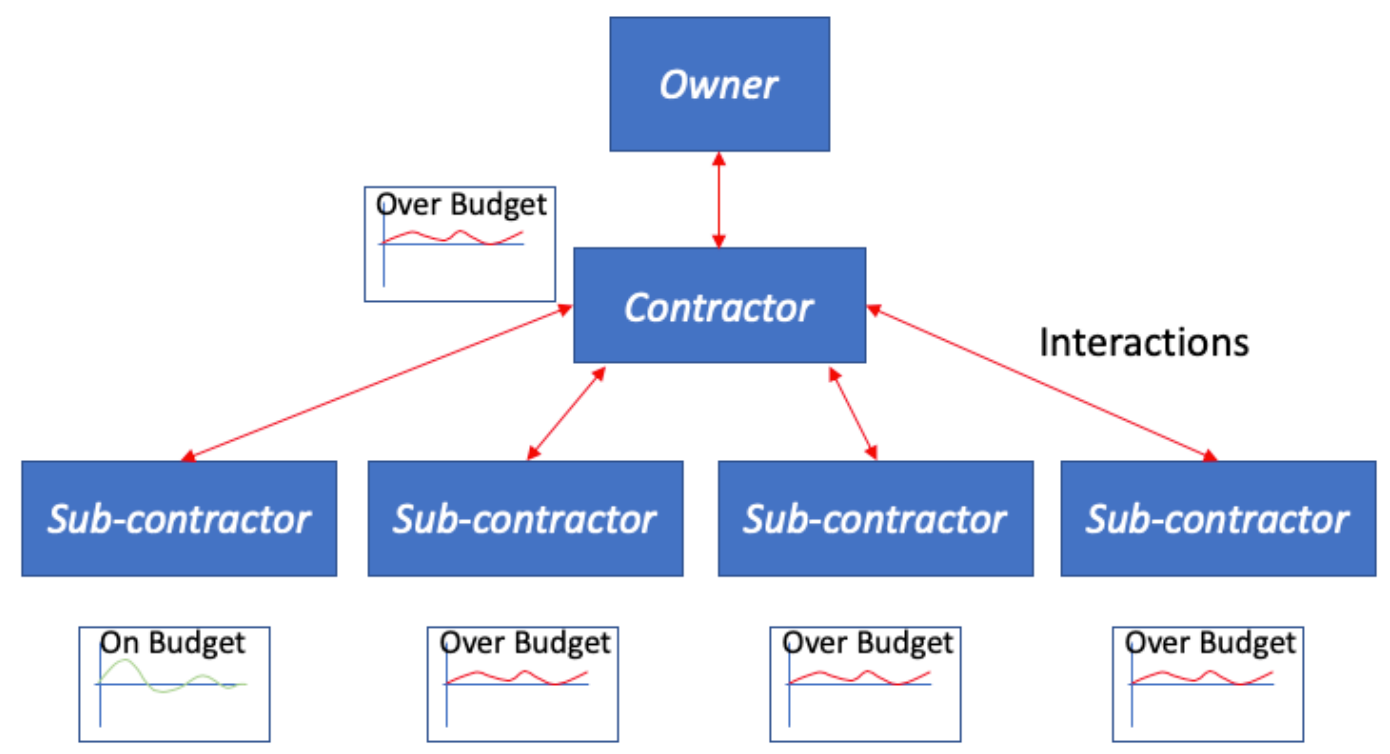

Figure 25: Interactions between contractor, owner, and sub-contractors 
Consolidating these interactions (i.e., reducing the number of sub-contractors) can reduce the number of degrees of freedom in the system and thus the complexity of the project. This approach to assessing complexity of a project also has the advantage of emphasizing the structure of the organization as a function of the number of driving interactions rather than just the overall system size.

\subsection{Parallels in Other Systems}

There are several potential parallels of this work to systems in other fields. Controls and signal processing share a similar mathematical background with feedback, gain, control, and filtering. Geographical Information Systems also have parallels with the idea of disequilibrium described in this dissertation and through the use of symmetry. These parallels are not addressed in this dissertation but are potential points of departure for future work.

The field of network stability also uses much of the same language as project management when discussing disequilibrium and complexity. For example, Johansson and Hassel (2010) state that critical infrastructures "often have high degrees of complexity". Also there is discussion in the literature regarding interdependencies (Rinaldi 2004) between infrastructure components that translate well into this dissertation's discussion on component interactions.

This chapter has developed methods to represent systems visually and mathematically and analyze their interactions by number and nature. Next these methods and representations developed in this chapter will be applied to projects in chapter 4. 


\section{Complexity in Service System Operation}

Three case studies are used to illustrate the use of the metric for complexity. A distinction is made between systems that have pre-determined structure verses systems that are organized ad hoc. The former project involves planning that reflects the underlying domain structure. For example, in a construction project, there is a schedule outlining agreed upon start and end dates and a work breakdown structure that reflects the sequence and duration of tasks to be performed. Hence, a construction operation is represented using cycle diagrams and production rates are measured as a function of cycle times. This is in stark contrast to an emergency response that is typically an ad hoc operation with volunteer involvement and a structure that emerges in an unplanned way out of the needs and urgency of the moment. Planning and exercises do take place to develop the "symmetry", a general frame of reference for a type of incident. However, the actual situation on the ground sometimes lends itself to an ad hoc state of operation, where components operate based on the general planning guidelines that may be in place. In operations where there is little to no prior planning in place, components extensively rely on the experience of past events and exercises. The following case studies include a construction project involving pre-construction (project) planning. The two emergency response case studies are ad hoc events where the responders and incident management team rely on general emergency operation plans, but primarily on experience from previous events and exercises.

The reason for assessing both the planned and ad hoc organizations is to test if the following fundamental questions can be applied to both, and if so, what the conclusions can be drawn about the complexity from the mathematical analysis in the last chapter:

- Is there disequilibrium in the interactions and is this causing information flow failures?

- How is the measure of complexity applied in this case and what is that measure?

- Can the measure of complexity be used to reduce the complexity in the system?

\subsection{Construction Operations}

\subsubsection{Case Study: Rock River Project}

Using the methods and concepts of complexity and disequilibrium discussed in Chapter 3, this dissertation will analyze the case of the Morgan Street Bridge in Rockford, Illinois as an example of how reducing the number of interactions can reduce the complexity of a project.

\subsubsection{Project Background and Context}

The Morgan Street Bridge project replaced the aging 900-foot spandrel concrete arched vehicle bridge over the Rock River (Figure 26) in Rockford, Illinois. Originally built in 1890 to connect the east and west parts of Rock River, the replacement of the bridge was seen by the city as an opportunity to jumpstart the city's efforts for urban renewal (Prange 2013). Revitalizing neighborhoods on both sides of the bridge was an objective of the city and the bridge was seen as an integral component of the community. As the owner, the city solicited input from the community, believing that the bridge could serve as the centerpiece for community 
redevelopment. Input was solicited throughout the design and construction phases using a context-sensitive solution process. The project objectives, which are the project symmetry, developed through the input received from focus groups, interviews, and surveys were:

- Increase traffic capacity

- Improve safety for vehicles and pedestrians

- Improve mobility to both sides of the river (including pedestrians)

- Enhance aesthetics of the area

- Accommodate fishing from the bridge

- Anchor the redevelopment of the surrounding community

Based on the community input, the design selected for the replacement structure was a network tied arch bridge. Due to the cost estimate of the project (baseline cost was $\$ 21.8$ million), the Federal Highways Administration required a Value Engineering study (Prange 2013). The expectations of the study were low, as the project was considered a typical construction project with mostly known variables. The Value Engineering study team was composed of 14 individuals including members from the Illinois Department of Transportation, representatives from the city, utilities, railroad relocation specialists, construction specialists, and a Value Engineering facilitator. The study group was broken into five teams: Bridge, Railway, General, Design Suggestions, and an Out-of-Scope group. Each group was asked to develop proposals that would reduce costs or add to the objectives of the project (Prange 2013).

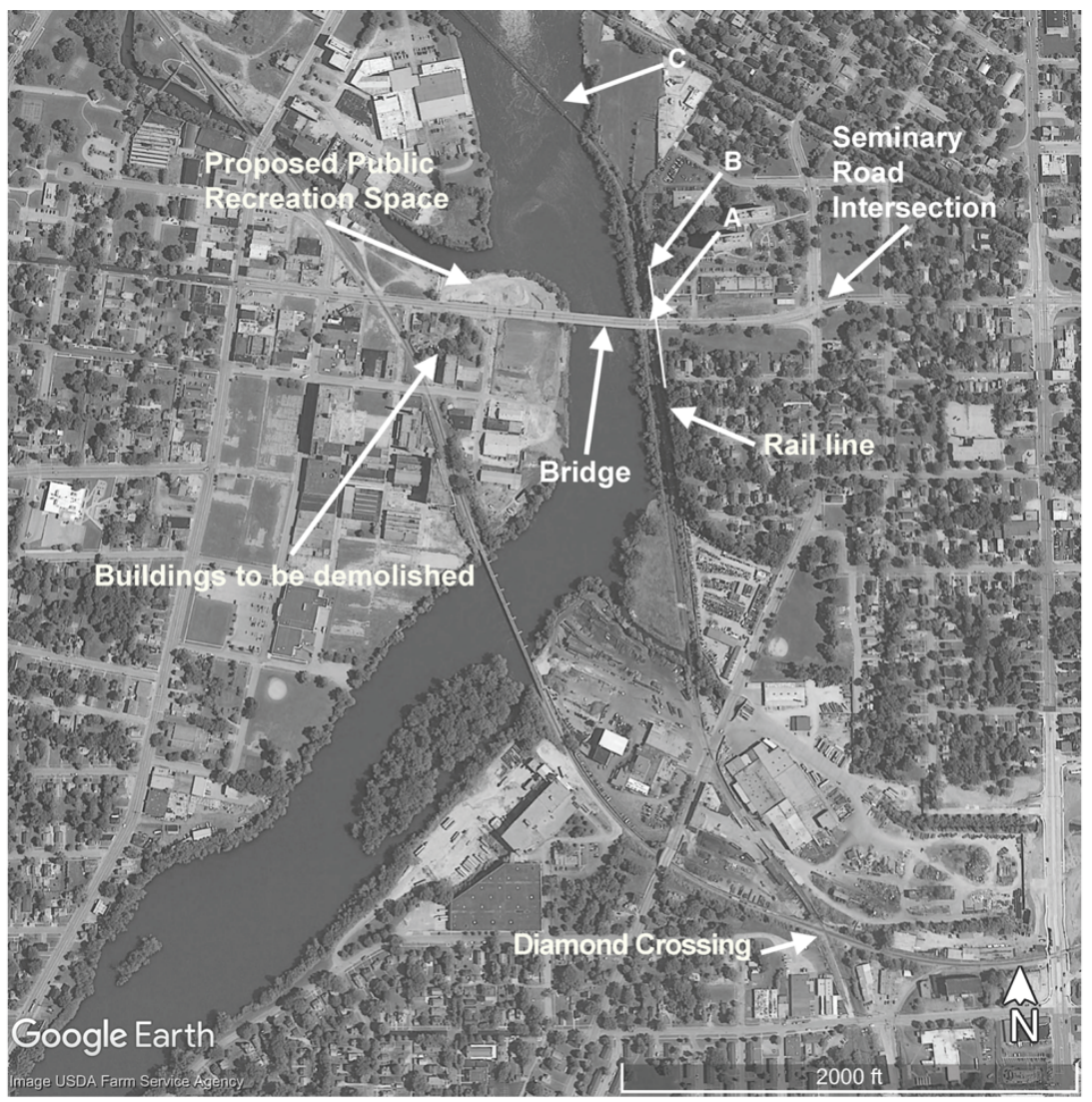

Figure 26: Map of project area 
The Value Engineering process took place during a two-day workshop. During the workshop, 78 proposals were created. After deliberation, the Value Engineering team recommended 11 proposals of which 9 proposals were accepted, resulting in savings to the project of $\$ 1.6$ million (Prange 2013). Three of the most unexpected proposals were:

1. Remove the rail line from under the bridge on the east embankment.

2. Reuse the concrete from bridge demolition (and from an additional project the city was undertaking nearby) for fill on the river embankment.

3. Add a roundabout at the Seminary Road intersection east of the bridge.

These three projects resulted in a savings of $\$ 1.26$ million and furthered the goals of the project (Prange 2013).

The first proposal was the result of an attempt to reduce the cost of maintaining rail operations during construction, which would involve using a flagger. After discussing the options, it was determined that the railroad companies involved would be willing to, and in fact had previously discussed, permanently rerouting the rail traffic. This allowed them to eliminate a difficult to maintain diamond crossing, and four at-grade crossings, and improve the safety and efficiency of rail operations (Prange 2013). The removal of the at-grade crossings also reduced traffic congestion in the city. The elimination of this single interaction between the bridge and the rail line (denoted by " $A$ " in Figure 26) allowed several other changes to take place in the project. These changes included, a redesigned bridge span of a 370-foot tied arch main span (rather than a 300 foot in the original design) with shorter approach plate girder spans, elimination of a retaining wall (denoted by "B" in Figure 26), a lower bridge profile, and others discussed below. The result was a savings of $\$ 815,000$ (Prange 2013). The recreational opportunities afforded by the removal of the rail line were the most significant. Now walking and biking trails could be added to the project, an existing rail bridge up stream of the project (denoted by " $\mathrm{C}$ " in Figure 26) could be repurposed into a pedestrian bridge, and greenspace with river front views and access could be added - all of which furthered the objectives of the project.

The removal of the rail line also allowed the bridge piers to be removed from the river. The initial design required the bridge piers to be located in the river due to the interference from the rail line under the east end of the bridge. There were concerns about construction method cost and long-term scour due to the river current. In addition, the removal of the piers had positive environmental impacts as the river contained several threatened species (Prange 2013).

The proposal for reuse of concrete from the bridge demolition was an attempt to both reduce environmental impacts and cut costs. Concrete from demolition would be used to build up the riverbank (north of the bridge area), which was to serve as a public recreational use resource, thus adding to the social vitality of the area. Reusing the old concrete would reduce the amount of fill that would be needed to be brought in from off-site. The city was already planning on the demolition of other buildings near the west approach to the bridge, and it was proposed that recycling the demolition materials from both the bridge and these other structures would save costs. This proposal saved $\$ 220,000$ (Prange 2013).

The roundabout project proposal was on a section of land east of the bridge approach. The land contained obsolete public housing units that the city was planning to remove as part of an urban 
renewal campaign (Prange 2013). This proposal was considered out of scope (and out of the construction limits), but the boundaries of consideration were expanded to include this area as doing so would improve traffic flow and reduce accidents - both of which were project objectives. It would also reduce costs compared to constructing and maintaining a standard signalized intersection, reduce noise and pollution (from idling vehicles at a signal), and be more aesthetic (Prange 2013).

\subsubsection{Interactions of the Project}

The project contains 5 bridge interactions defined by how the asset to be replaced was interacting directly with its immediate surroundings. They were as follows (see Figure 27: Interactions from the perspective of the bridge.):

1. Bridge-River

2. Bridge-Rail line

3. Bridge-Recreation use space

4. Bridge-Environment

5. Bridge-Corridor enhancement (Seminary Road)

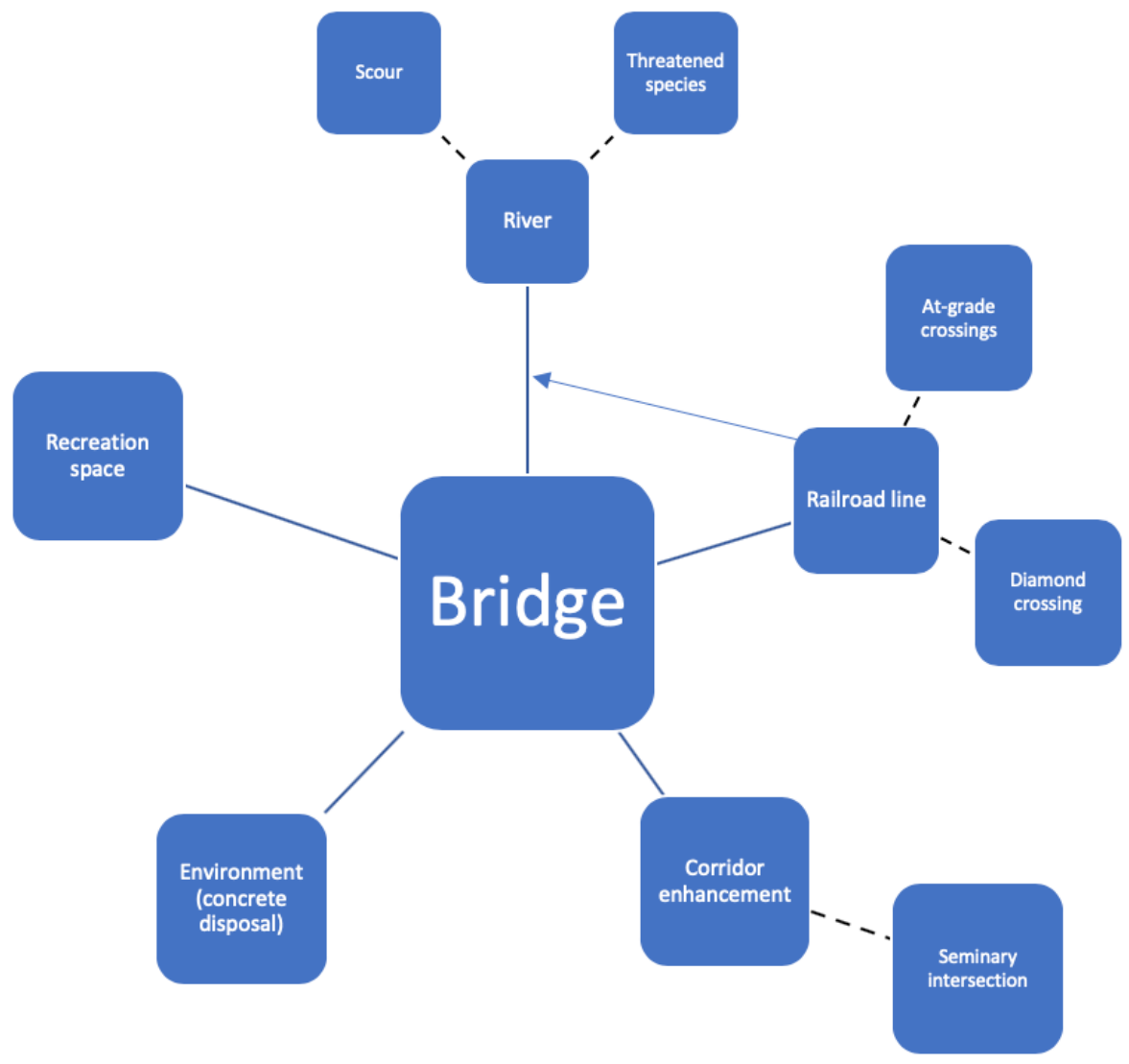

Figure 27: Interactions from the perspective of the bridge. 
The bridge interacted with the Illinois Railroad, which passed under the east approach. One of the significant costs of the construction plan was the cost of maintaining rail operations while the existing bridge was removed, and the new bridge constructed above the rail line (Prange 2013). Another interaction was between the bridge and the corridor enhancement - which was directly impacted by the Seminary Road intersection to the east. This interaction impacted traffic flow and volumes. There were two interactions between the bridge and the environment, waste from demolition and new bridge piers in the river. Concrete from the demolition was to be removed from the site and disposed. The bridge piers were going to be placed in the river, impacting the river flow, environment, and the river would also impact the piers and complicate construction.

\subsubsection{The Driving Interaction and Disequilibrium}

Due to the river velocity, the potential for scour, and the cost of construction, the preferred design was to remove the piers from the water and place them on each shore (Prange 2013). However, due to the proximity of the rail line to the river, there was not sufficient space for both the pier and the railroad. The rail line would also create a safety issue during construction and be expensive. During a Value Engineering study, a discussion on how to reduce the cost of rail traffic flagging during construction lead to the determination that the railroad line could be removed entirely. This allowed the piers to be located out of the river, and the track removal allowed the abandoned rail grade to be put to recreational uses. The creation of recreational opportunities was a major objective of the project. The track reconfiguration also allowed the removal of a railroad diamond further along the track and several other at-grade crossings.

\subsubsection{Complexity Analysis}

The perspective, or area of interest (the symmetry), is important to the analysis. This analysis is bridge centric as the bridge is the main component of the project, meaning each primary interaction is directly interacting with the bridge. However, it could be community revitalization centric, for example, and would have a different number of interactions.

Prior to the removal of the rail line, there were 5 bridge interactions, with 5 secondary or chained interactions. Secondary interactions are defined as interactions that are not directly connected to the bridge but can still have a secondary impact through (Figure 28). For example, the bridge interacts with the river through the bridge piers, and the river causes scour on the piers. This means there are 5 degrees of freedom or states of the system (see Table 2). This research has defined the number of interactions from a perspective as the metric of system complexity, therefore, the complexity of this system is 5 . 


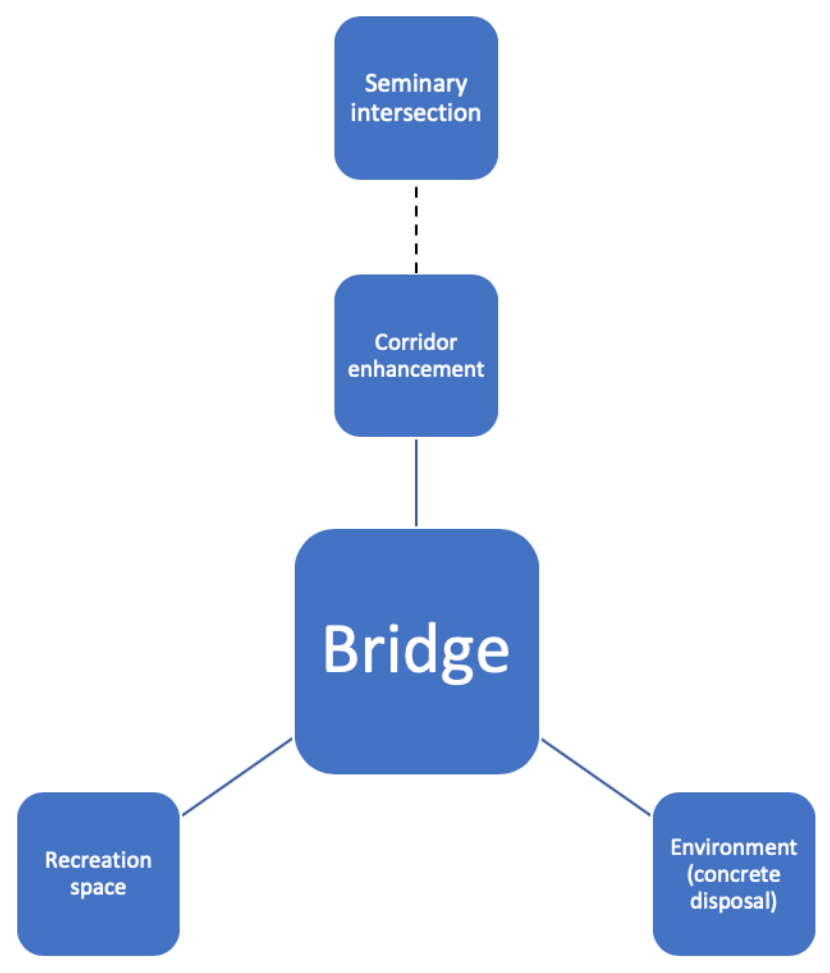

Figure 28: Interactions without the rail line. The solid lines are primary interactions, and the dashed line indicates a secondary interaction.

Table 2: Complexity

\begin{tabular}{lccc}
\hline & Project Interactions & $\begin{array}{c}\text { Bridge } \\
\text { Interactions }\end{array}$ & $\begin{array}{c}\text { Complexity } \\
\text { Measure }\end{array}$ \\
\hline Original Plan & 10 & 5 & 5 \\
$\begin{array}{l}\text { After rail line } \\
\text { removed (Value }\end{array}$ & 4 & 3 & 3 \\
Engineered plan) & & &
\end{tabular}

Removing the rail line from the project reduces the number of project interactions to 4 , with 3 of those directly impacting the bridge. This means there are 4 total interactions and a complexity of 3 for the system. As there are fewer states in which the system can exist, the complexity of the project is reduced.

\subsubsection{Summary}

By reducing the bridge interactions of the project from 5 in the original plan to 3 in the Value Engineered plan, the complexity of the project was reduced. Critically, this reduction in 
interactions does not reduce the primary objectives of the project - quite the opposite, reducing the interaction of the bridge and the rail line increased opportunities to better align the plan with the project objectives (the symmetry). The correlation to the Crutchfield and Young (1989) definition of complexity, is that project becomes more reducible with limited "information loss" when better aligned with the project objectives. This illustrates the importance of identifying the driving interactions of a project determining the disequilibrium in that interaction, the level of complexity, and how to reduce critical interactions to reduce the system complexity.

\subsection{Emergency Management}

This section discusses complexity through the lens of emergency response (a special case of project management). This section begins with a background in emergency response concepts and operations.

\subsubsection{General Case:}

The concepts of complexity are examined through the lens of emergency response. Using the definition from Crutchfield and Young (1989), we can define the symmetry as a "normal condition" (no emergency). Or it can be defined as the "normal" or "planned" incident response. The difference between the actual condition and the symmetry is the disequilibrium. The disequilibrium is detected in a system through dynamic feedback. This can be thought of as the system trying to correct itself (return to the mean). However, when the feedback is sufficient, the feedback can bring about a phase transition in the system causing the system to settle at a new equilibrium (if the system is stable). During an emergency response, the Incident Action Plan (IAP) is an outline of the "normal" response (the symmetry). However, as the event progresses, deviations from the "normal" response (due to differing initial conditions, resource availability, etc.) are the disequilibrium. The Incident Commander makes adjustments based on this disequilibrium using the dynamic feedback (the information flow coming back from the responders on the ground). However, if the disequilibrium is sufficiently large, a drastic change in the response plan (IAP) may be necessary (Bigley and Roberts 2001). This can take the shape of a change in mission objectives or may require a change in tactics. Miller (1956) and Flood (1987) above discussed information volumes. When overloaded, responders began to chunk information, packing it into larger pieces but sacrificing detail. This loss of information is the result of the disequilibrium in the system.

This research has discussed complexity through as the number and nature of interactions. This dissertation will now discuss this scaling through the lens of emergency response to determine the impact information flow.

\subsubsection{ICS Structure and Scaling}

ICS has a hierarchical structure based on functions performed during the incident response. The structure uses an Incident Commander (or a group consisting of a commander from each responding agency or level of government which form a "Unified Command") as the manager of the response (Bigley and Roberts 2001). This dissertation will use Incident Commander and 
Unified Command interchangeably as they perform the same functions (however, a Unified Command does have the potential for disequilibrium between its members). Under the Incident Commander, there are four "General Staff" positions (Operations, Logistics, Planning, Finance/Administration), each led by a Section Chief (see Figure 29) (Bigley and Roberts 2001; Texas A\&M Engineering Extension Service (TEEX) 2016). This is very different from how some agencies respond in a routine emergency where they are normally organized as a standalone organization that must deal with all of the General Staff functions themselves. In ICS, the decision makers at the top of the structure (Incident Commander) formulate the objectives and policies of the incident response while the responders assigned under the General Staff positions implement the tactics devised at the General Staff level (Anderson et al. 2004). The structure has been examined by Moynihan (2009), who concluded that ICS is a "mode of network governance, akin to a network administrative organization (NAO)" where an external centralized coordinator is charged with organizing the response of multiple independent organizations.

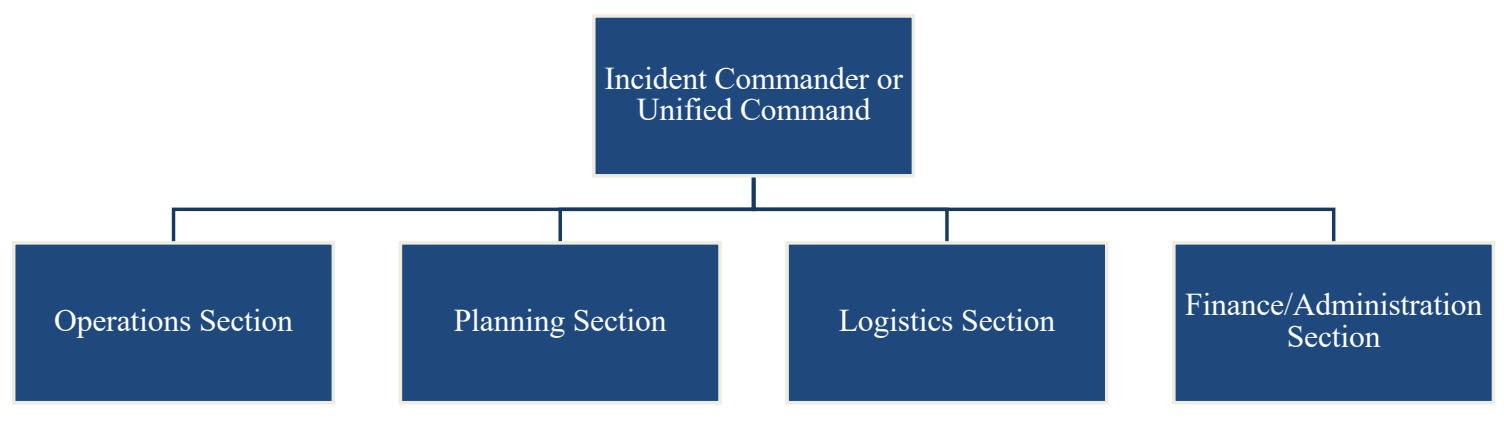

Figure 29: Generic Incident Command Structure

As mentioned previously, the Incident Command System structure is scalable. This is accomplished by only assigning General Staff positions when that section's activities become too burdensome for the Incident Commander to manage alone (Bigley and Roberts 2001). Two examples are used to illustrate scaling: first, a traffic stop with one police officer. Second, a multivehicle crash involving three police officers, a fire department, and medical first responders.

In the first example, a single car is pulled over for speeding. The police officer serves as the Incident Commander as well as all of the General Staff positions (Figure 30). The officer determines the objectives of the stop, as the Incident Commander, based on a "typical" behavior for someone pulled over at a traffic stop. This mental model or Incident Action Plan is the symmetry of the system. The methods and tactics to accomplish the objectives are determined, as the Operations Section Chief. The officer gathers information and develops the Incident Action Plan, as the Planning Section Chief. As the Logistics Section Chief, the officer determines the communications needs and what equipment will be needed for the stop. And the officer documents the resources used, and the time required for the stop as the Finance/Administration Section Chief. As the stop progresses, the officer may learn new information (after running the vehicle license plate and learning the vehicle is not registered for example). Based on this new information (dynamic feedback), the "typical" stop may now be in disequilibrium. The officer 
makes adjustments (perhaps asking for additional information from the driver) and reassesses the situation to see if those adjustments reduces the disequilibrium.

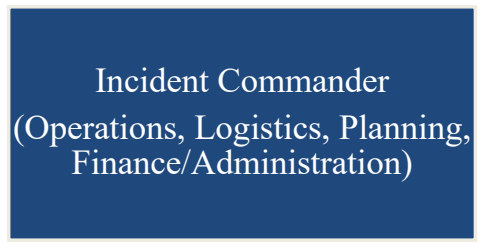

Figure 30: Example ICS structure for a single traffic stop incident

In the second example, four cars are involved in an accident on slippery roads. Three police officers are dispatched as well as a fire department and medical first responders.

The Fire Chief is the Incident Commander on the scene (Figure 31). The initial reported conditions (from the 911 call) will be the basis of the mental model of the incident that the responders will have- their symmetry. After arriving on scene, the first step is a scene size-up to determine what the objectives are (broadly, these are life safety, property preservation, environmental protection). The initial mental model is adjusted based on this information (dynamic feedback). The difference between the initial mental model and the new model is the disequilibrium. They assign Police Officer 1 as the Planning Section Chief to document the accident and conduct interviews (gather information). The tactical operations of law enforcement, fire, and medical services will be overseen by the Operations Section Chief, but because the role is unassigned, it falls to the Incident Commander to fulfill the role of Operations Section Chief. The Operations Section Chief (the Incident Commander in this example) then assigns a "Traffic Control Team" consisting of one police officer with two fire fighters to manage traffic control. Then, medical first responders are assigned to see to the medical needs of the occupants of the vehicles involved in the crash, and a firefighting team is assigned to perform victim extrication from the vehicles and fire protection duties. The Incident Commander then asks the dispatcher (at the 911 Center) to document the resources used and the time each unit arrived and departed the scene, making the Dispatcher the Finance/Administration Section Chief. As the Logistics Section Chief is not assigned (in addition to the Operations Section Chief), the Incident Commander performs the duties of those section chiefs. With the assignments made to meet the objectives of the incident, the system enters a new equilibrium, and the disequilibrium drops to near zero. However, the situation is dynamic. Perhaps the condition of one of the victims changes. This is a change in disequilibrium. There is some information loss as the Incident Commander may not be the first informed (the medical responders will attend to the victim first and inform the IC when the situation is stabilized). This short time interval has both disequilibrium (a change in the situation from the plan), and information loss because it takes time to inform the IC that there is a change. There is also dynamic feedback to this new information (the responders treat the victim differently). If the initial responders arrive on scene and determine that there is a hazardous materials spill, the large disequilibrium creates a phase transition. The system begins to work towards a new equilibrium. In this case, the information loss occurs prior to the disequilibrium. The example illustrates how the Incident Command System can expand or contract to meet the needs of the incident and how the characteristics of complexity are present in emergency 
response. The need to make decisions based on incomplete information is a frequent feature of emergency response (Yu and Lai 2011).

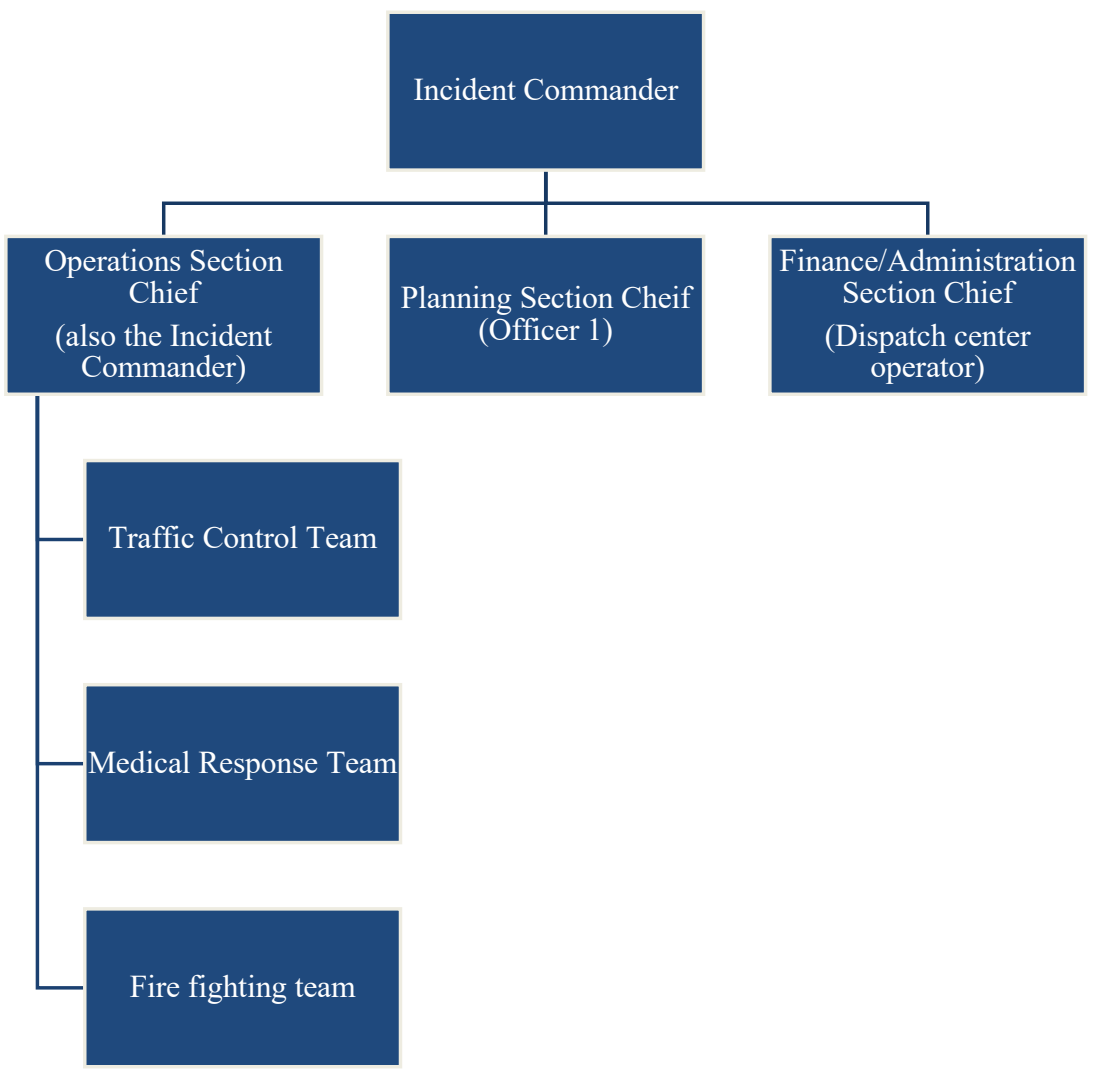

Figure 31: Example ICS structure for a multi-vehicle traffic accident

Returning to the question of minimum disequilibrium: Can the disequilibrium ever reduce to zero? With different agencies and multiple responders, there is always some information loss between them, and this information loss represents disequilibrium. The dynamic feedback to this constant background disequilibrium is indicated by comments about preferences in communication: "Can you give me an update every 15 minutes?" "Please change to talkgroup 31EMER for all traffic so I can hear what's going on." Contributions of disequilibrium include a number of issues of emergency response identified by Oomes and Neef (2005) and Comfort et al. (2004): 1) timecriticality: does the incident management scale fast enough to manage the responders and resources arriving at the incident; 2) multidisciplinary nature of responders, and if they can communicate and work in teams; 3 ) multiple layers of the response leading to the question "Who's in command?"; and 4) how to maintain situational awareness. The Incident Command System works well in addressing three of these concerns. First, the issue of time-criticality of response scale-up is addressed by the Incident Commander's ability to appoint General Staff positions as needed, allowing the incident management to scale-up quickly. Second, the multidisciplinary nature of (many) responses is addressed by two components of ICS: Unified Command, and common operating framework. Having a Unified Command allows a representative of the lead responding agencies to have buy-in with the response objectives and planning. Responders are therefore not "taking orders" from other agencies, but rather their own 
people. Having a common framework for the response (ICS) that is mandated across the United States by FEMA allows all responding agencies to know what to expect before they arrive on scene (reducing disequilibrium). Third, the issue of "Who is in command?" is addressed by specifying that the command will be kept at the lowest possible level of government (usually the county or city) for an incident. When additional assistance is needed from higher levels of government (state or federal), this assistance is requested in the form of resource requests (materials, responders, incident management teams, etc.). The question of situational awareness is about information flow, and not well addressed in ICS but is a critical in terms of disequilibrium.

\subsubsection{Information Flow}

While the scaling flexibility of the Incident Command System is well documented, the scaling of the information flow within the ICS is not (Bigley and Roberts 2001, Oomes and Neef 2005). As emergencies present in different sizes, the information flow for the incident must scale. As an incident grows, the first obstacle is scaling from the Incident Commander, who is also the acting Section Chief for all four sections, to having four Section Chiefs the Incident Commander must now communicate with via feedback loops (giving instructions and receiving information from field units). The success of the response is dependent on the ability for information flow to scale with the ICS structure in an appropriate amount of time (Oomes and Neef 2005). In the above example of a simple traffic stop, most of the information flow is internal to the officer. However, the officer may request information from outside such as requesting a check on the driver's history from a dispatcher (Figure 32). The delay in the system comes from waiting for this information to be returned before the officer can refine the Incident Action Plan (i.e., what they are going to do). In the multi-vehicle accident example, as the ICS has scaled up, the information flow has grown more complicated, and as a result, the number of delays in the information flow path has increased (Figure 33).

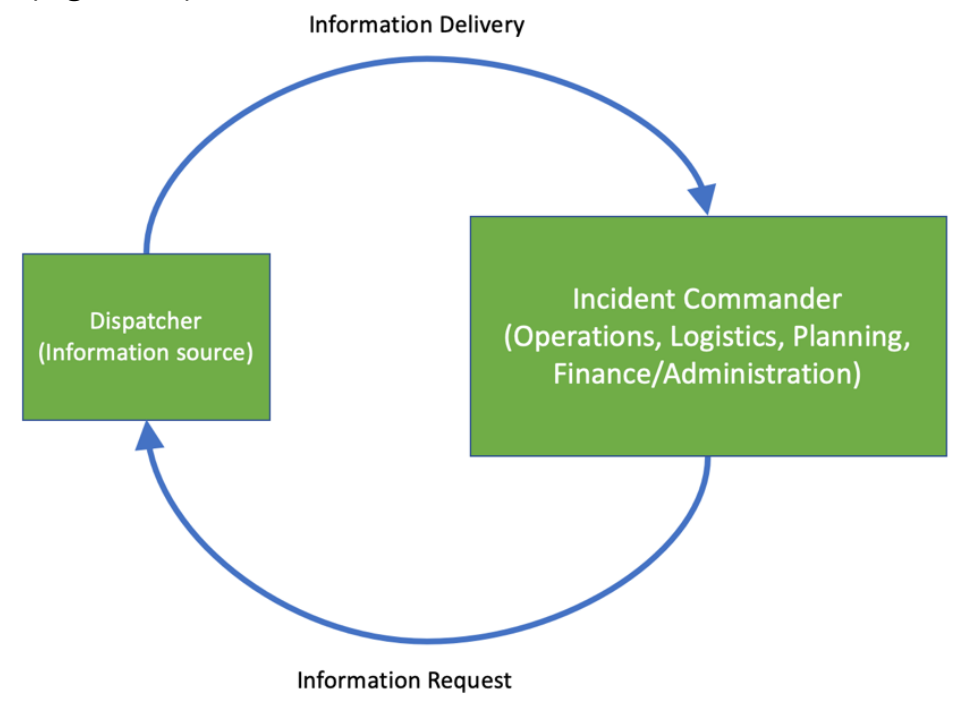

Figure 32: Information flow for a single traffic stop incident. The delay introduced is the Information Request/ information Delivery feedback loop. 
Many of the incident management problems, identified by Moynihan (2009) and discussed above, are fundamentally information flow problems - responders not knowing where they fit into the response structure, differing or unclear mission objectives or tasks, difficulty determining who is part of the formal response structure and who is not (especially with volunteer organizations), and responders not knowing the capabilities of other responder agencies (Moynihan 2009). Delays in the information flow can exacerbate these problems. Comfort et al. (2011) states the makeup and diversity of the components impact the information flow, as specific needs and abilities for information flow may vary, further adding to the disequilibrium.

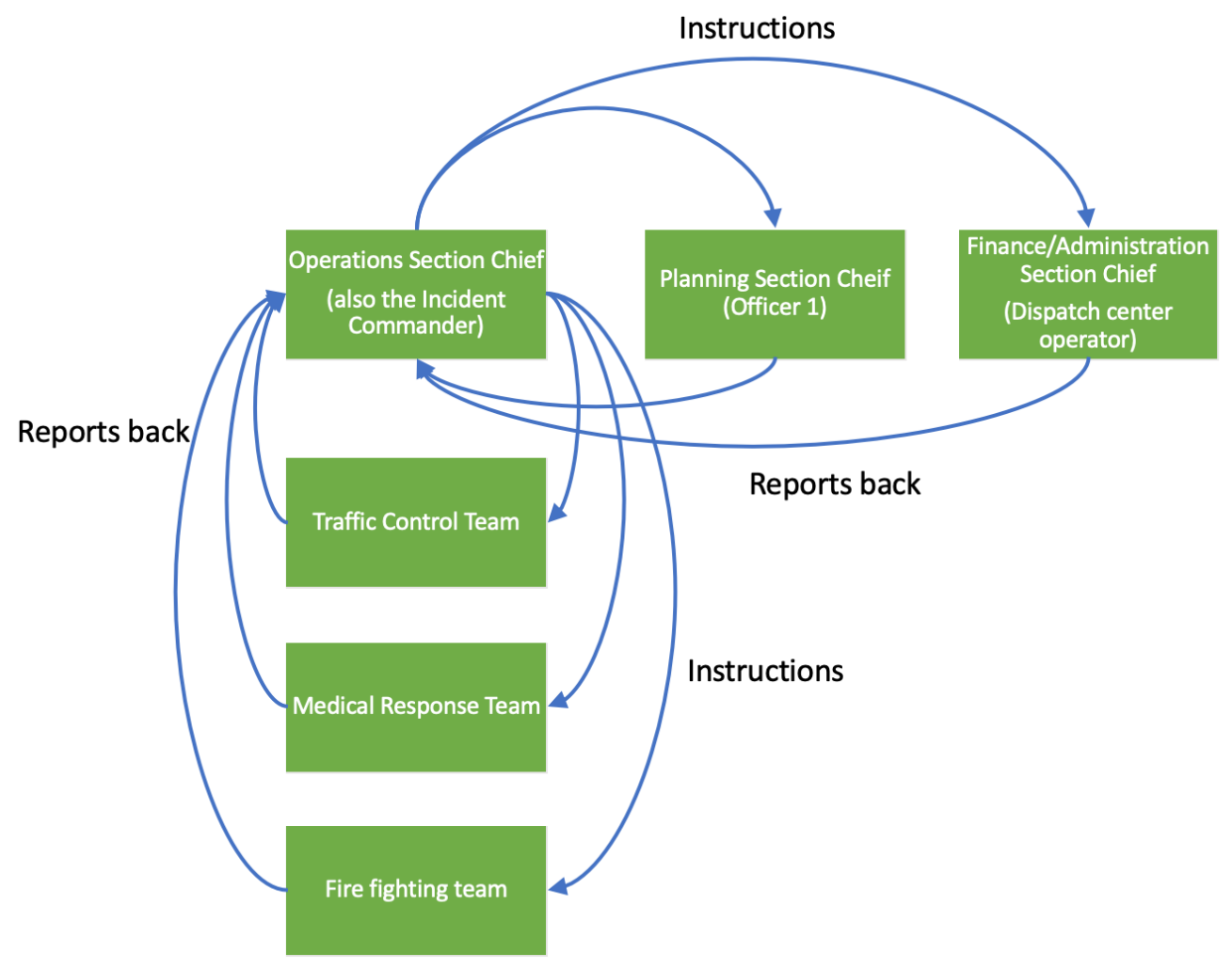

Figure 33: Information flow for multi-vehicle traffic accident

Work by Comfort et al. (2004) examined information quantity, quality, and flow, noting that a lack of important information at critical moments can significantly impact the response. Comfort et al. (2004) found that as more useful information was available and communicated to responding agencies, the efficiency of the response improved. The initial report of the incident can be incomplete or inaccurate, causing the first responders to arrive on scene unprepared for the existing conditions (large disequilibrium). The information between the first responders and the dispatch center may not be clear due to improper communication procedures. Dispatchers may confuse agencies with one another or may not communicate with other dispatchers clearly, such as information being misinterpreted at the call center. Information from the dispatch center to the Incident Commander may not flow or be inaccurate due to the multiple information transactions such as those listed above. Additionally, information from one responding agency to 
another is also a source of failure with agencies not communicating the necessary information in a timely manner. In addition to these lapses in human communication flow, there are also technological communication issues such as incompatible, unmaintained, or insufficient quantities of equipment. Many first responding groups are volunteers and may lack sufficient training. Some responding groups choose to not implement ICS. Therefore, working with other responders more difficult as there is no common operating framework. Simpson and Hancock 2009) note in their review of emergency response literature that the common thread in emergency management is working in a disorganized environment. Using graph theory to locate cut points in the information network, Comfort et al. (2004) identified critical nodes that, if removed, could cause information islands in a network, or teams that did not receive or transmit the information that was required to perform their tasks or allow decision makers to give further instructions. All of these issues contribute to the disequilibrium of the system.

The path, timing, and quantity, and accuracy of information passed through the ICS structure will help dictate the effectiveness of the emergency response. When information is not readily available (delay), the disequilibrium increases. A balance point between incoming information to decision makers (typically the Incident Commander) and the information passed on as assignments to teams in the field, must be found to provide the most effective information flow. Too much information coming into decision makers overwhelms them and risks important information being missed (Manoj et al. 2007). Insufficient information or information arriving too late, prevents appropriate assignments for teams. A lack of information flow between the Incident Commander and the Operations Section Chief will add another step in the communication chain, adding additional delay to the system and increasing disequilibrium.

Fundamentally, communication problems in emergency response are information flow problems. As mentioned previously, combining the ideas from Crutchfield and Young (1989) and López-Ruiz et al. (1995) implies that information loss is an indication of disequilibrium. For example, disequilibrium is the difference between the Incident Action Plan (IAP) and the actual conditions on the ground. The IAP is a model of the situation and will be informed and adjusted based on the dynamic feedback received by the Incident Commander from the responders.

Next, two emergency response cases will be presented to demonstrate how the measure of system complexity can be applied and complexity can be reduced by reducing the number of interactions within the response system.

\subsubsection{Case Study: Houghton Search}

The following case study is from a missing person search in Houghton, Michigan in August of 2019. It is an example of how the proposed metric of complexity can be applied to reduce system complexity. The study discusses the interactions between the components and the disequilibrium present in the form of information flow failures.

\subsubsection{Background}

An 82-year-old woman with dementia left her home in west Houghton approximately 13:00 on Saturday, August 17, 2019. Her family searched the area until approximately 16:00 when they 
called the Houghton Police Department. The Houghton Police searched the area until 18:20, when they contacted Superior Search and Rescue for assistance. Search and Rescue arrived at 18:50 and began sending out search teams. The Michigan DNR fire-spotting plane was diverted to the area and searched from the air until dark. A private citizen also took his airplane up and searched from the air until dark. Searching on the ground in the woods continued until dark. Then searching on the roads (and around buildings) continued until 1:00 on August 18, 2019 when the search was paused and scheduled to resume at 8:00.

During the night, a request was made for Civil Air Patrol (CAP) air assets to the Air Force Rescue Coordination Center and was approved by the Michigan State Police. CAP was scheduled to begin flying at 7:00.

At 8:00, Superior Search and Rescue resumed the search with assistance from several local fire departments, law enforcement agencies, and medical first responders. Additional volunteers began arriving and by 11:00 were overwhelming the staging area. At the peak, there were nearly 160 volunteers.

At 14:35, a team reported finding the subject. Michigan Tech EMS was dispatched to the scene to assess and extract the subject. The woman was about 20 feet uphill of a snowmobile trail (in an area that had been searched several times) under a tree root ball. She was conscious and had no obvious injuries. She was transported to the hospital. Search teams were recalled and demobilized.

\subsubsection{Search Operations}

The searching process is based on cycles, different for each components of the search. These are described for several components below.

Incident Commander/Unified Command Team (Figure 34) activity cycle:

1. Develops the initial Incident Action Plan (based on initial information available)

2. Holds tactics meeting with the Operations Section Chief

3. Begins planning next operational period (next steps) with Planning Section Chief

4. Reviews information coming back from the field (relayed from Operations Section Chief or Planning Section Chief).

5. Updates Incident Action Plan with new information

6. Repeats steps 2-5. 


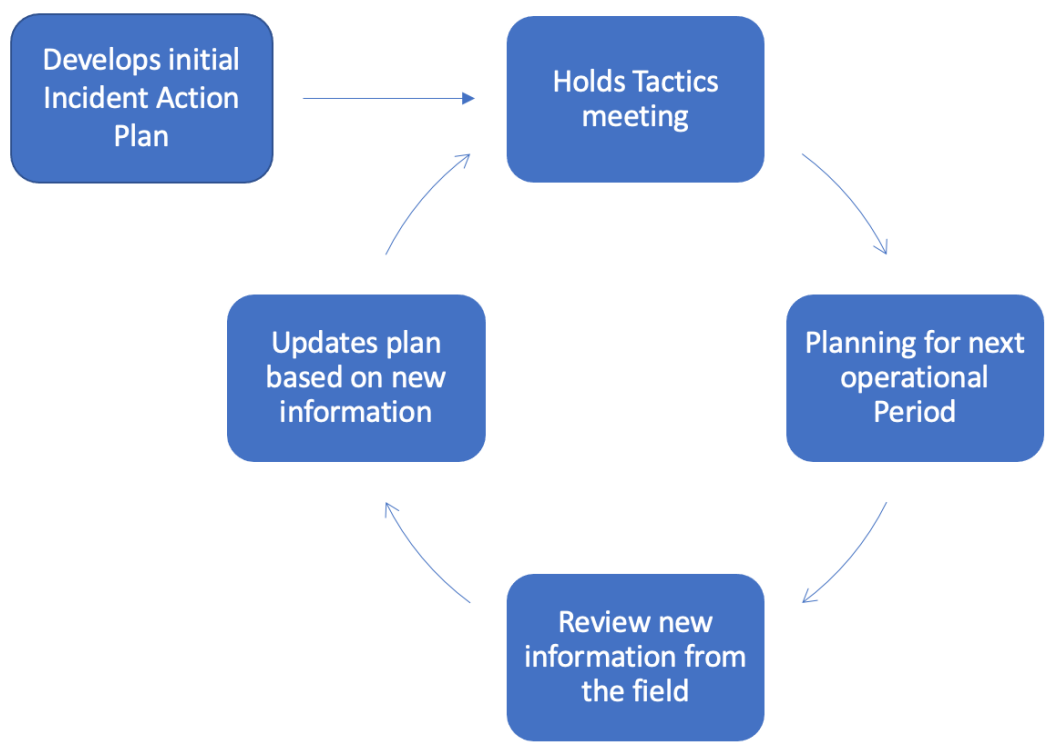

Figure 34: Incident Commander/Unified Command Operational Cycle

Operations Section Chief (Figure 35) activity cycle:

1. Holds tactics meeting with Incident Commander/Unified Command

2. Develops assignments for teams

3. Briefs teams on assignments and receives debriefing information from teams

4. Develops next assignment for teams

5. Repeats steps 1-5

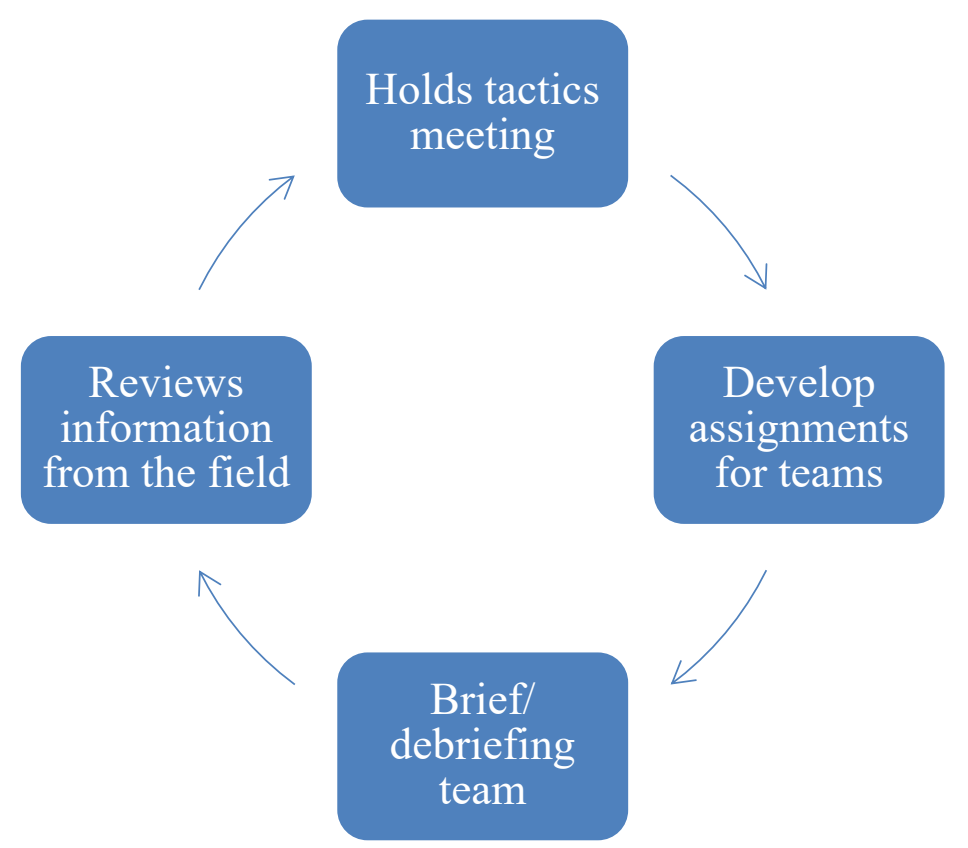

Figure 35: Operations Section Chief Cycle 
Search Team (Figure 36) activity cycle:

1. Briefs from Operations Section Chief to get assignment

2. Leaves staging on deployment (transportation)

3. Searches in the field (carrying out assignment)

4. Returns to staging (transportation)

5. Repeats steps 1-5

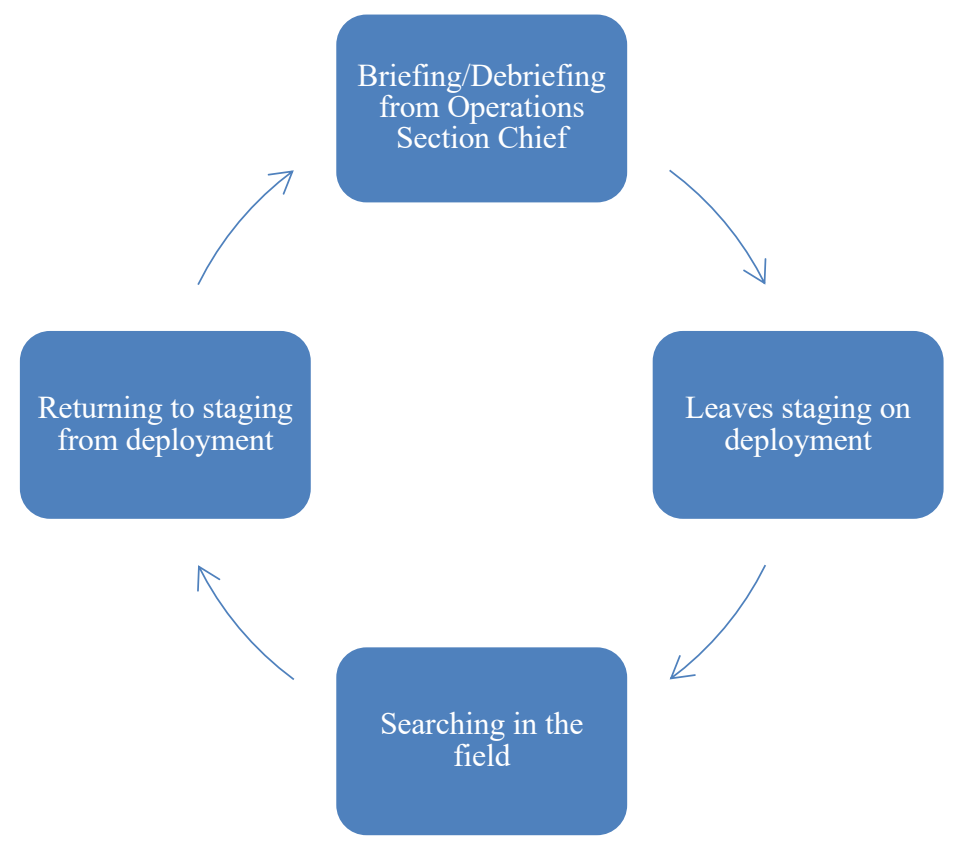

Figure 36: Search team cycle

Each of these cycles is linked together. In Figure 34 and Figure 35, for example, the Incident Commander and the Operations Section Chief are linked through the tactics meeting. The Operations Section Chief and the Search Teams are linked through the briefing/debriefing in Figure 35 and Figure 36. These linkages are the interactions in the overall system and can be seen in the combined system in Figure 37. 


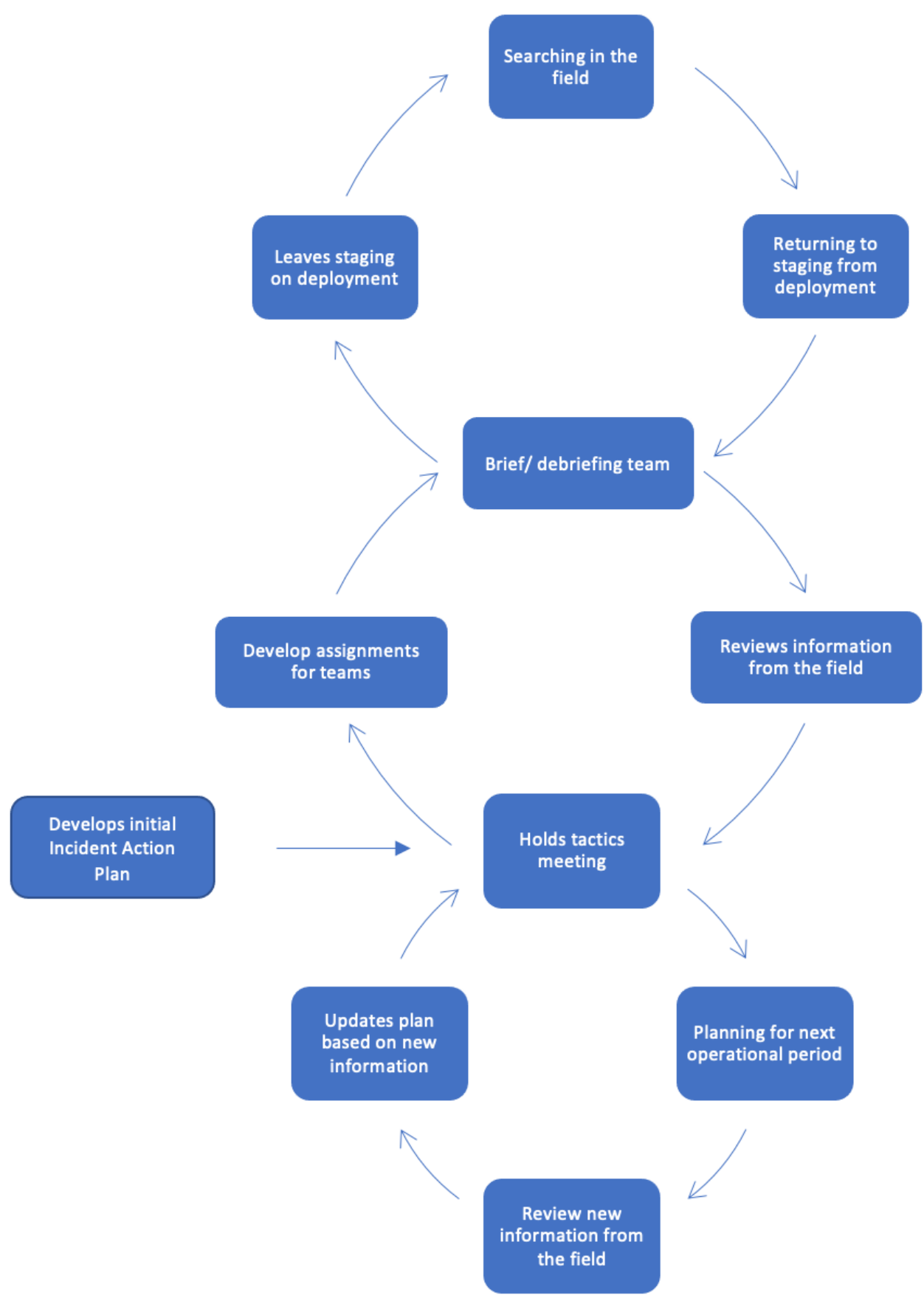

Figure 37: Interactions between component cycles

\subsubsection{Interactions}

The interactions associated with this search are broken into two parts the initial operational period (the first day of the search) and the second operational period (second day of the search). As mentioned previously, the structure of an emergency response is fluid and scales up and down as needed. Therefore the interactions scale and reorganize as the incident progresses. 
Initial Operational period (based on Figure 38):

- Incident Commander with:

- Operations Section Chief

- Law Enforcement

- Operations Section Chief with:

- Search teams 1 and 2

- Law Enforcement with:

O Officers 1 and 2

○ K9 Unit

- Dispatch Center

Second Operational Period (based on Figure 39):

- Incident Commander with:

- Operations Section Chief

- Planning Section Chief

- Logistics Section Chief

- Law Enforcement

- Emergency Medical Services (EMS)

- Operations Section Chief with:

- Search teams 1-10

- Staging Manager

- Medical First Responders

- Civil Air Patrol

- Law Enforcement with:

O Officers 1, 2, 3

○ K9 Unit

- Dispatch Center

- Planning Section Chief with:

- Documentation group

- Staging Manager

- Operations Section Chief

- Logistics Section Chief with:

- Transportation providers

- Food providers

- Communication support team

\subsubsection{Areas of Disequilibrium}

Disequilibrium was present in the search on a number of levels:

- Information flow: The following information flow issues occurred during the incident:

- Sometimes information was not relayed to or from the Incident Commander due to the Incident Commander being occupied with other tasks

- The number of teams during the second operation period became unmanageable for the Operations Section Chief to debrief and process all of the information fast 
enough to update assignments efficiently. The result of this was a delay where teams were waiting for their next assignment.

- Due to many of the searchers being volunteers from the community with no previous searching or emergency response experience, there was a significant gap (disequilibrium) in their knowledge of search operations, safety protocols, and expectations. Field training was provided, but further slowed the planning and assignment process.

- Information from subject's family: Information about the subject was relayed to the Incident Commander through law enforcement. This information was verbal and incomplete. For example, the subjects last name was misunderstood (and relayed to searchers as the wrong name, which the was not corrected for the Incident Commander until the second operation period.

- Weather: The weather was a contributing factor to information flow failures. There was heavy rain and wind during most of the second operational period. This limited the effectiveness of air support from Civil Air Patrol, made team briefings and debriefings difficult to hear, and significantly impacted the information transfer of assignments and team debriefing information all of which is done on paper and using paper maps.

- Search cycle: Due to the large numbers of search teams involved in the response during the second operational period, the search cycles outlined above in Figure 35 and Figure 36 had to occur more quickly than they would normally occur in order to minimize the waiting (and likelihood of angry confrontations) of volunteers.

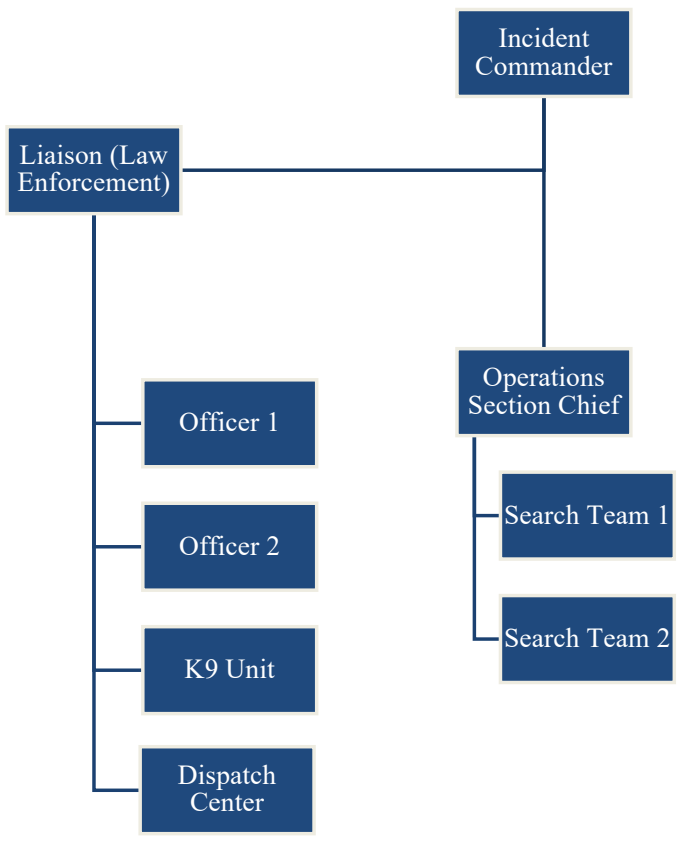

Figure 38: Initial operational period organizational chart 


\subsubsection{Measurement of Complexity}

The organizational structure of the response is shown in Figure 38 and Figure 39. As discussed in the Incident Command System structure above, the structure of the response changed as the incident progressed and the response scaled.

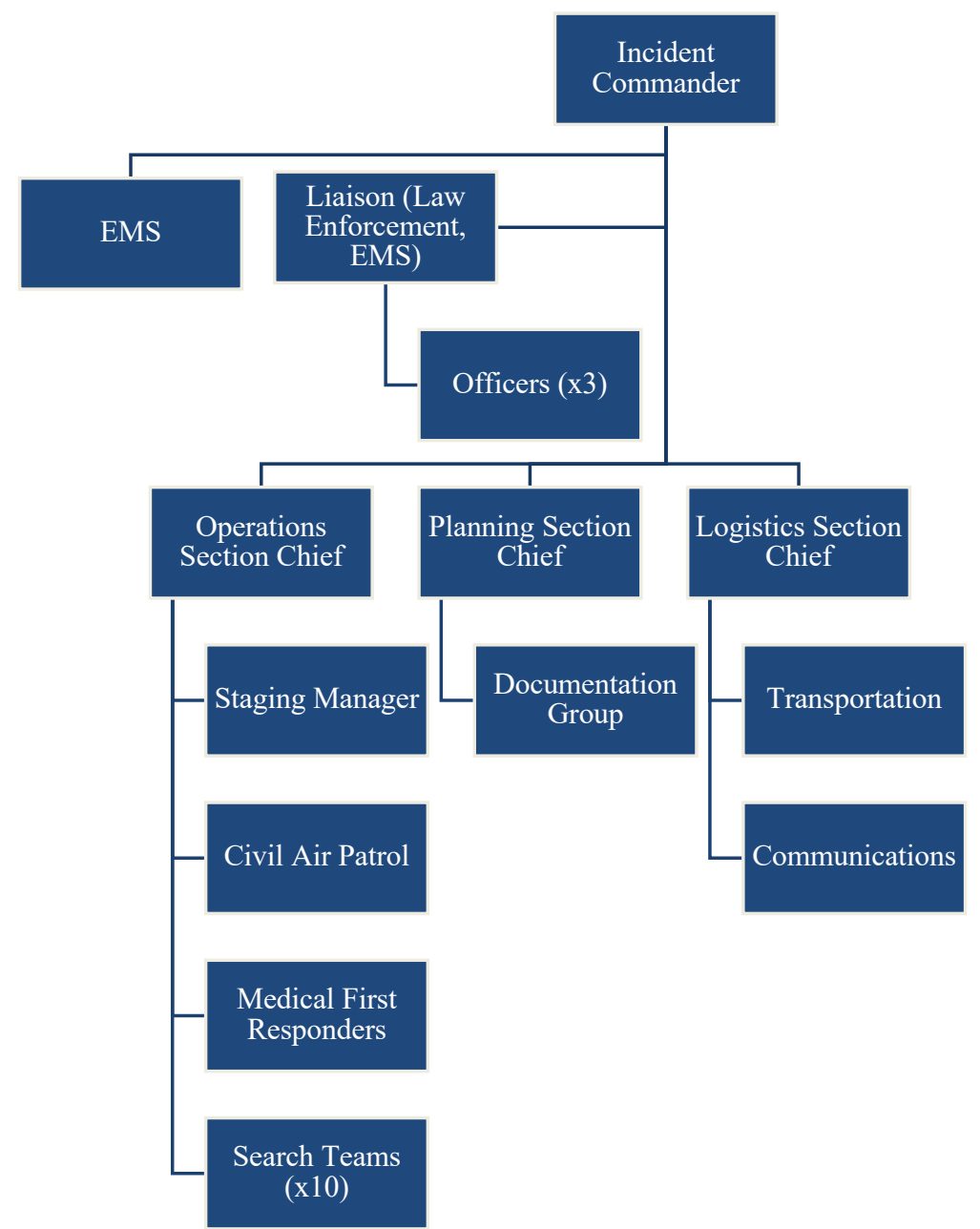

Figure 39: Second operational period organizational chart.

As discussed above, the simplified cycle diagram in Figure 40 shows how the search information flow moves through the operation. Information flow is two-way and occurs at predetermined intervals. The Incident Commander develops the incident objectives and passes this information on to the Operations Section Chief. The Operations Section Chief reports the status of the 
objectives back to the Incident Commander. There is only one Incident Commander and one Operations Section Chief.

Next, the Operations Section Chief develops the tactics to accomplish the incident objectives and assigns search teams to carry out those tactics. The search teams then debrief with the Operations Section chief upon completing their assignments. For a search there could be multiple search teams that interact with the Operations Section Chief.

Each Search Team is composed of members. Each member has specific tasks assigned to them to complete as part of the search. These can include navigation, radio operation, etc. In a typical search, each team is composed of 4-7 people. Although for this search, teams varied in size from 3 to 15 people at various points during the incident.

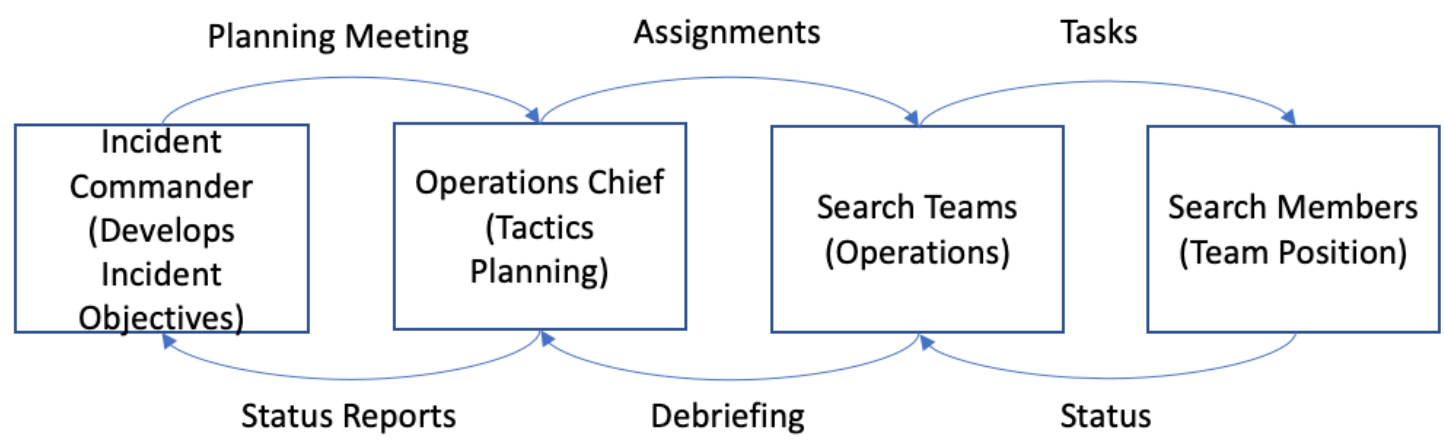

Figure 40: Information flow cycle diagram for a search.

Converting the cycle diagram to a circular diagram shows the interactions at each level of the organization. Figure 41 shows how the interactions occur as the incident progresses. The circles rotate inside each other as time moves forward. Each dot represents an interaction between a circle and the circle that encloses it.

Next, the waveforms of this search are calculated. For the relationship between the Operation Section Chief and the search teams, because the number of teams was constantly changing for this analysis 9 teams will be used. However, there will be a delay added representing the delay resulting from overcrowding the Operations Section Chief with too many teams. The result is a circular representation shown in Figure 42 rather than Figure 41. Each team will also be shown with 4 people.

Therefore, the parameters are:

$$
\begin{aligned}
& n_{0}=1 \text {, Incident Commander } \\
& n_{1}=1 \text {, Operations Section Chief } \\
& n_{2}=9 \text { (teams per Operations Section Chief) }
\end{aligned}
$$


$n_{3}=4$ members per team

$\varepsilon=0.0833$, the delay in the search teams receiving their briefing or debriefing, equating to 5 minutes.

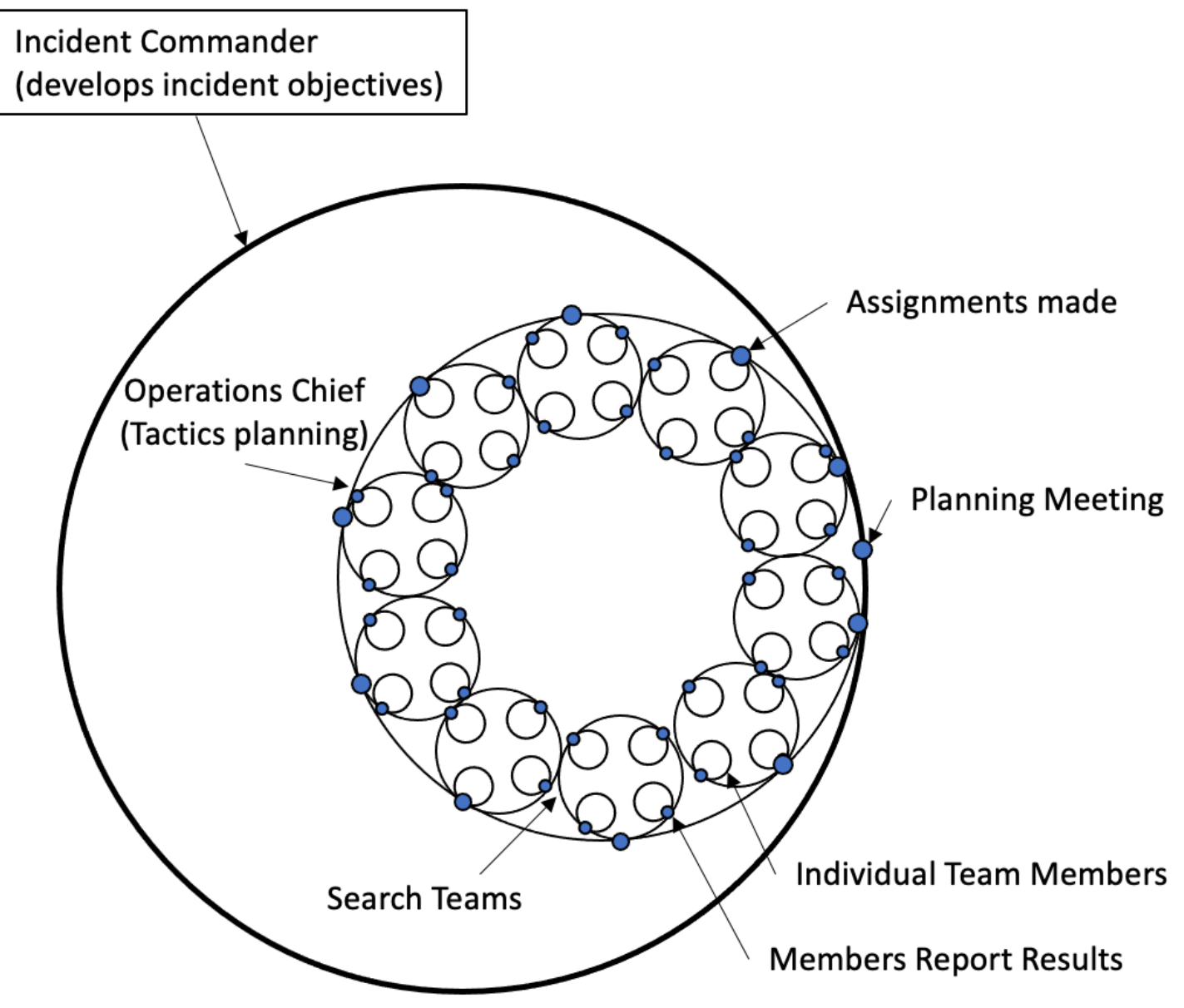

Figure 41: Circular representation of the search operations for a balanced system, showing the various levels of organization and the multiple Search Teams and members. 


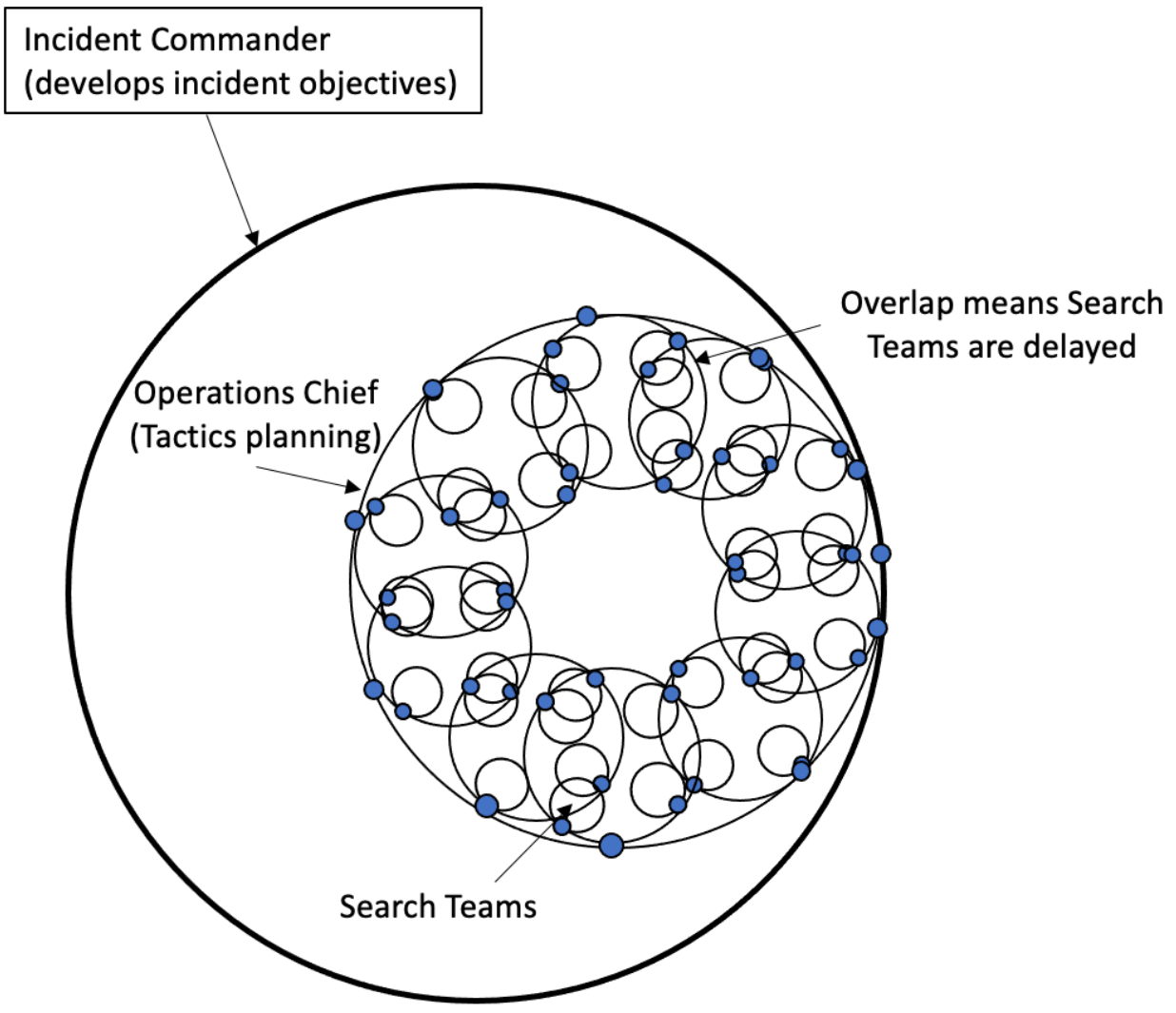

Figure 42: Unbalanced search circular representation of the search system.

The waveforms of each component are shown in

Figure 43. The frequencies and delay are shown in each plot. The Incident Commander and Operations Section Chief have the same waveforms, indicating that interaction is in balance. However, the Search Team waveform has a delay every cycle (while the team waits for their briefing or debriefing) indicating this is an unbalanced interaction. 

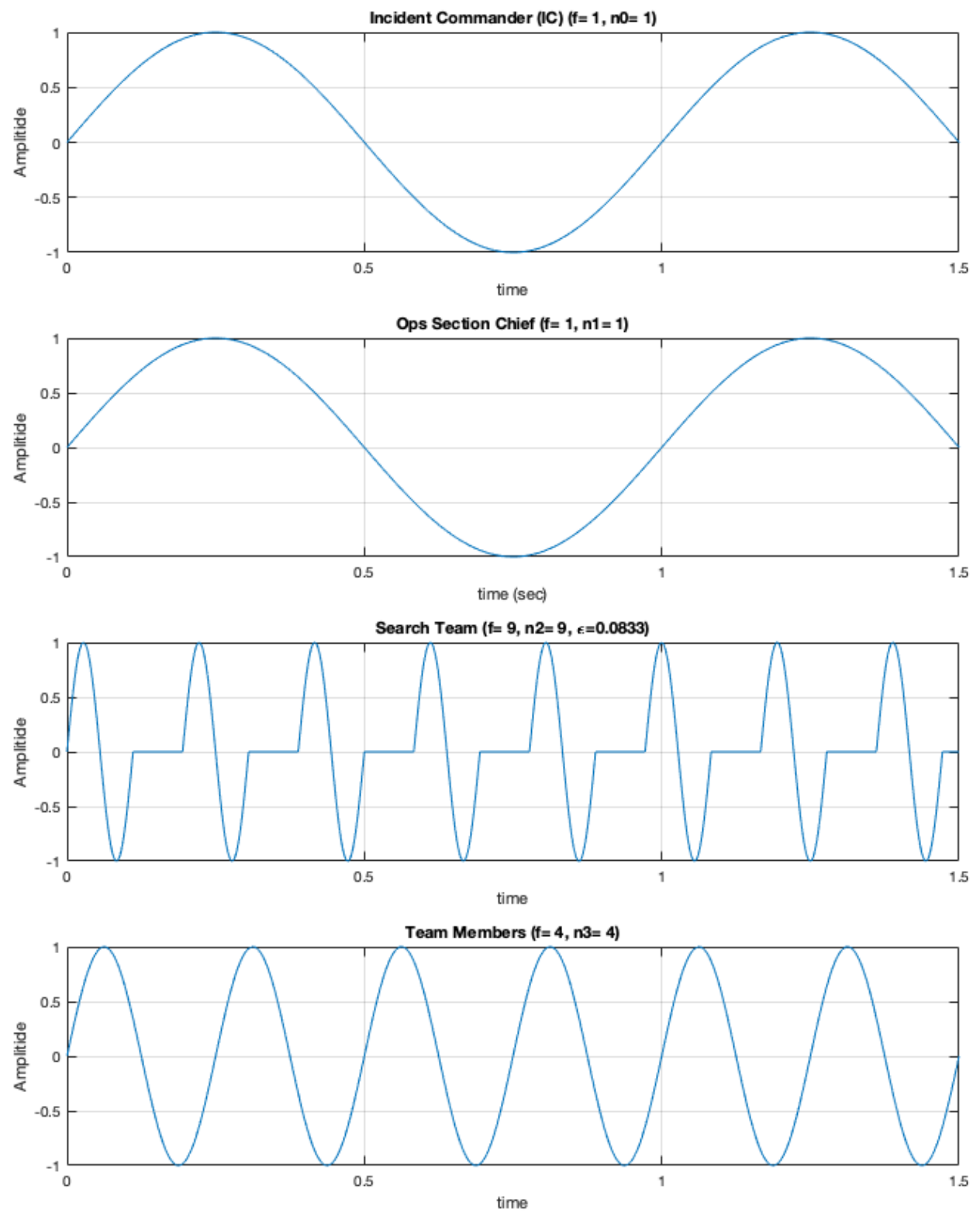

Figure 43: Component waveforms from Houghton Search.

The results (Figure 44) show that the three waveform frequencies are present in the FFT of the summation of the four components. However, as the Incident Commander and the Operations Section Chief both have the same frequency, the magnitude of that frequency (1 CPT) is twice that of the other frequencies present, indicating there are two components at that frequency. 

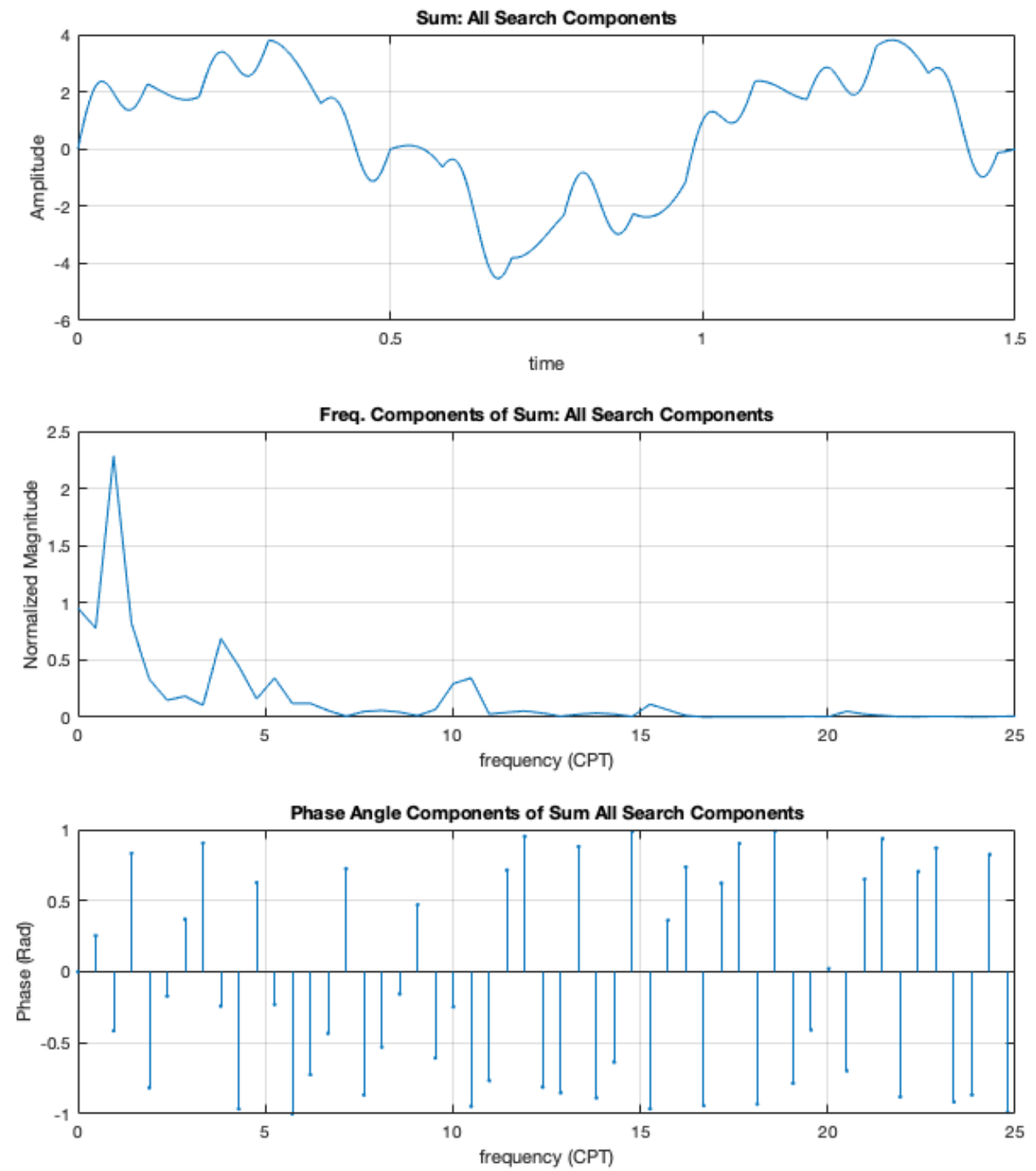

Figure 44: Summation of the four SAR component waveforms and the FFT of the sum, showing the magnitude and phase angle.

\subsubsection{Driving Interaction}

The driving interaction, or limiting interaction, for this incident was the interaction between the Operations Section Chief and the search teams. Due to the volume of volunteers, many of whom had no search or emergency response experience, there was disequilibrium in the form of delay in assignment creation and information loss due to abbreviated briefings and debriefings which contributed to the complexity of the system. The Incident Command General Staff noted the number of volunteers was increasing rapidly, assigned a Deputy Operation's Chief and added responsibilities to the Staging Manager (Figure 45). These additional people assisted with 
organizing, training, and briefing volunteers which reduced the number of interactions seen by the Operations Section Chief (Table 3).

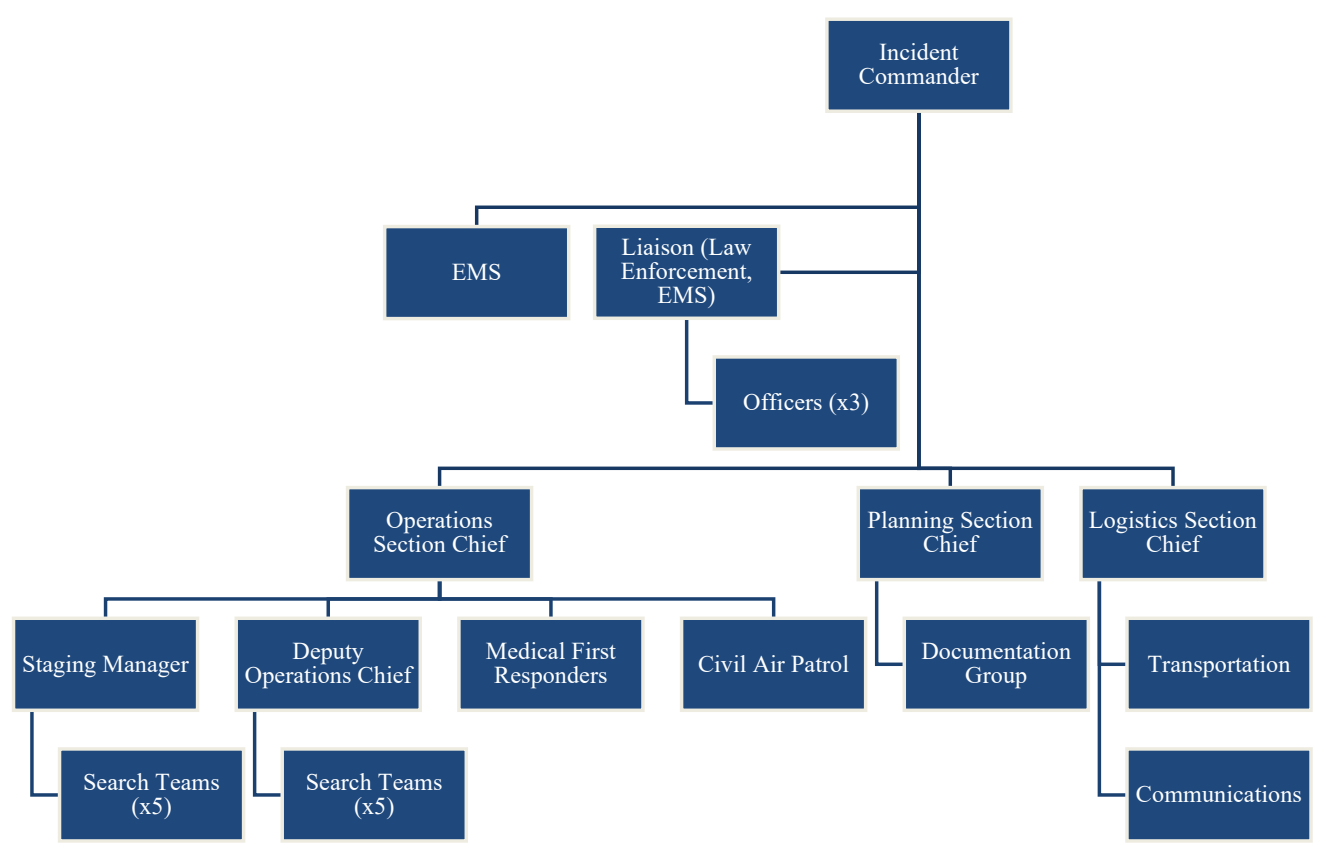

Figure 45: Second operational period (after additional staff added) organizational chart.

Table 3: Complexity by operational period

\begin{tabular}{lccc}
\hline Operational Period & $\begin{array}{c}\text { ICS } \\
\text { Interactions }\end{array}$ & $\begin{array}{c}\text { Operations Chief } \\
\text { Interactions }\end{array}$ & $\begin{array}{c}\text { Complexity } \\
\text { Measure }\end{array}$ \\
\hline $\mathbf{1}$ (initial) & 8 & 2 & 2 \\
$\mathbf{2}$ & 24 & 15 & 15 \\
$\mathbf{2}$ (after additional & 25 & 7 & 7 \\
staff) & & &
\end{tabular}

\subsubsection{Summary}

While ultimately successful, the disequilibrium present in several aspects of the Houghton Search impacted the efficiency of the response. While steps were taken during the incident to reduce the disequilibrium, the result was the total number of system interactions increased due to adding additional positions for the purpose of reducing delays in search team briefings and debriefings. While this increased the complexity, it reduced the disequilibrium. 
This case study reinforces the concept that using the number of interactions as a measure of complexity is really the potential for disequilibrium failures, but it is not a given that an increase in complexity will increase the disequilibrium in a system. This case continues to reinforce the importance of identifying the driving interaction and the disequilibrium in that interaction.

\subsubsection{Case Study: 2018 Western Upper Peninsula Flooding}

The largest case study of this dissertation is from the 2018 flash flooding in Houghton County, Michigan. The value of this case study is that it is a larger response than the previous case of the Houghton Search, both in terms of responders and the geographic area. It also involved components from outside the area at both the state and federal level with all of the coordination required to adhere to the protocols of the state and federal government.

\subsubsection{Background}

On June 17, 2018, central Houghton County endured a significant rain event that dropped approximately 6.7 inches of rain in a 6-8 hour period (Figure 46), classified by the National Weather Service as a 750 to 1000 year rain event. The result was catastrophic flash flooding beginning at 4:35AM over the highest populated portions of the county causing large scale infrastructure and personal property damage in excess of $\$ 120$ million.

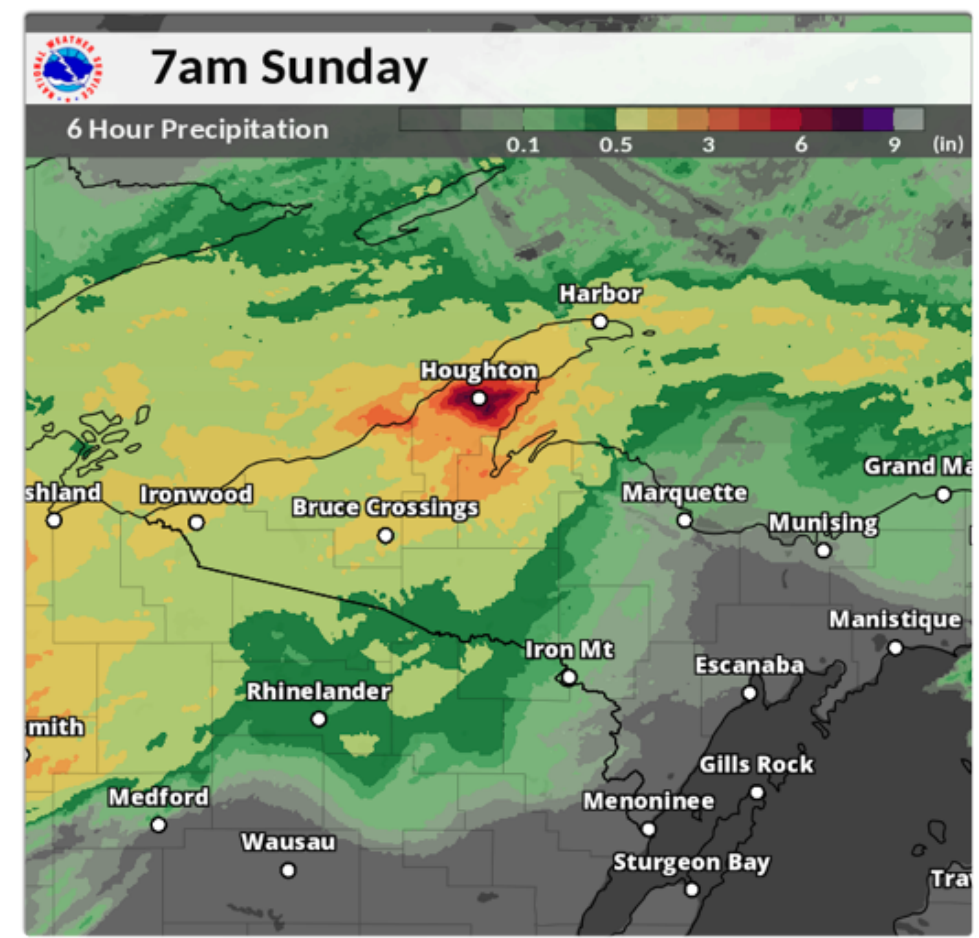

Figure 46: 6-hour Precipitation total: 1AM-7AM, June 17, 2018. Source: NOAA

Local resources were almost immediately overwhelmed. Additional assistance was requested from the State of Michigan through a request for a State Disaster Declaration, which was granted. 
The State Disaster Declaration opened up the availability for state resources to assist with the local effort. All of the state resources were organized through the State Emergency Operations Center (located 500 miles away). While additional resources were critical to the response, those resources also came with a nearly doubling of the number of people (components) that were now interacting with the response system. This significantly strained the information flow in the response at the Incident Command level.

While significant damage was done to the road system in the county, there was also significant but localized damage to homes. Approximately 468 homes were impacted with three destroyed and 50 with major damage. FEMA estimated the individual damage estimate to be $\$ 2,908,095$ (Federal Emergency Management Agency 2018b). Cleanup and repairs were organized through a Volunteer Resource Center (VRC) where homeowners could call and request volunteers to help clean out homes or yards. More than 2,131 volunteers (about $6 \%$ of the county population) helped with hundreds of projects including: digging mud out of basements, moving rocks out of front yards, debris removal, and transportation of equipment. While a Presidential Disaster Declaration was received for the Public Assistance (DR-4381-MI), a federal declaration for Individual Assistance was not approved, leaving homeowners without financial assistance from the FEMA.

The response can be divided into four stages: initial, stabilization, repair, and recovery. With each stage having a different organizational structure and its own set of interactions. This dissertation will examine primarily the initial and stabilization phases of the incident as they involved the most interactions and information flow issues.

The structure of the initial response (Figure 47) involved almost exclusively local entities. Following a modified ICS structure, the Unified Command consisted of the Houghton County Emergency Management Coordinator and the Houghton County Sheriff. The structure essentially had each entity report directly to the Unified Commanders (while the County does employ the FEMA ICS structure, there were very few people present that were familiar with the ICS structure at the time of the initial phase). This very quickly overwhelmed the Unified Command's capacity to receive, process, and transmit information without having the agencies wait. As the incident was still unfolding, decisions about evacuations, public warning, and rescue priorities were needed. This had a significant impact on the response.

As the incident moved from the initial into the stabilization phase (after the first 12-16 hours), the Unified Command added an incident management specialist from the Michigan Department of Energy, Great Lakes, and Environment (EGLE) to assist with the response. Additionally, recognizing the structure was unmanageable, a more formal ICS structure was setup (Figure 48). Section Chiefs were selected to serve as contact points in order to group agencies together. This reduced the critical interactions the Unified Commanders were required to maintain and the delay agencies were experiencing under the initial response structure. 


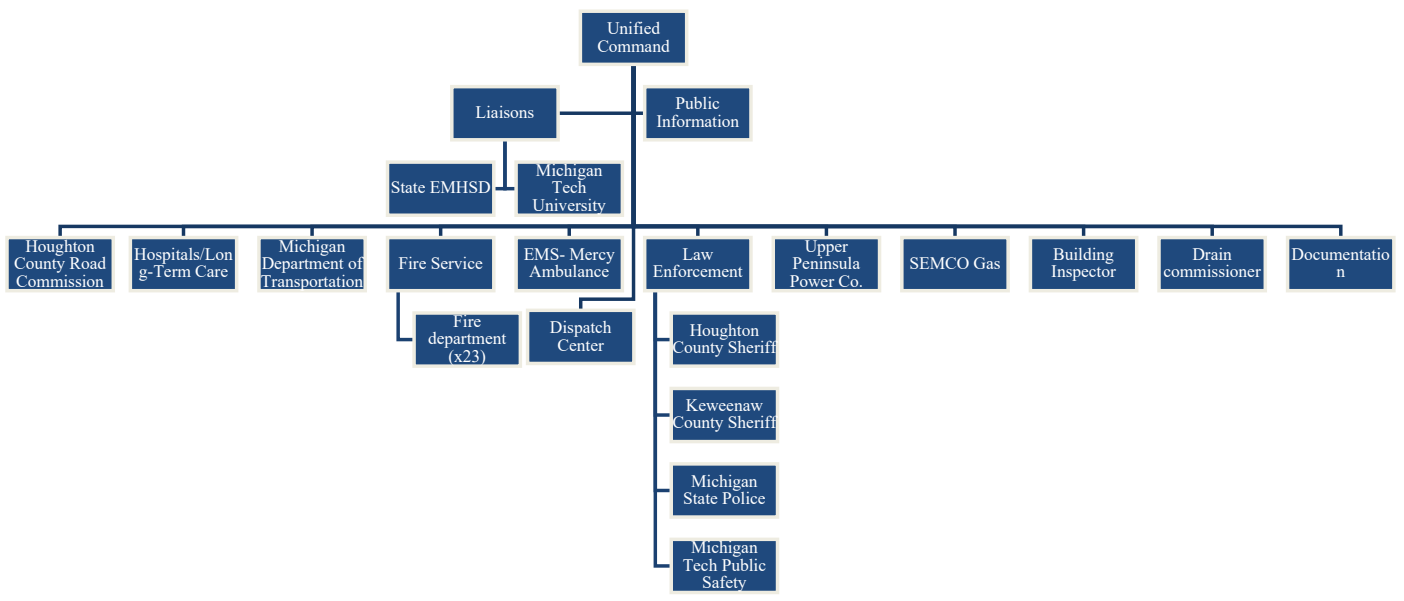

Figure 47: Initial Phase Organizational Structure

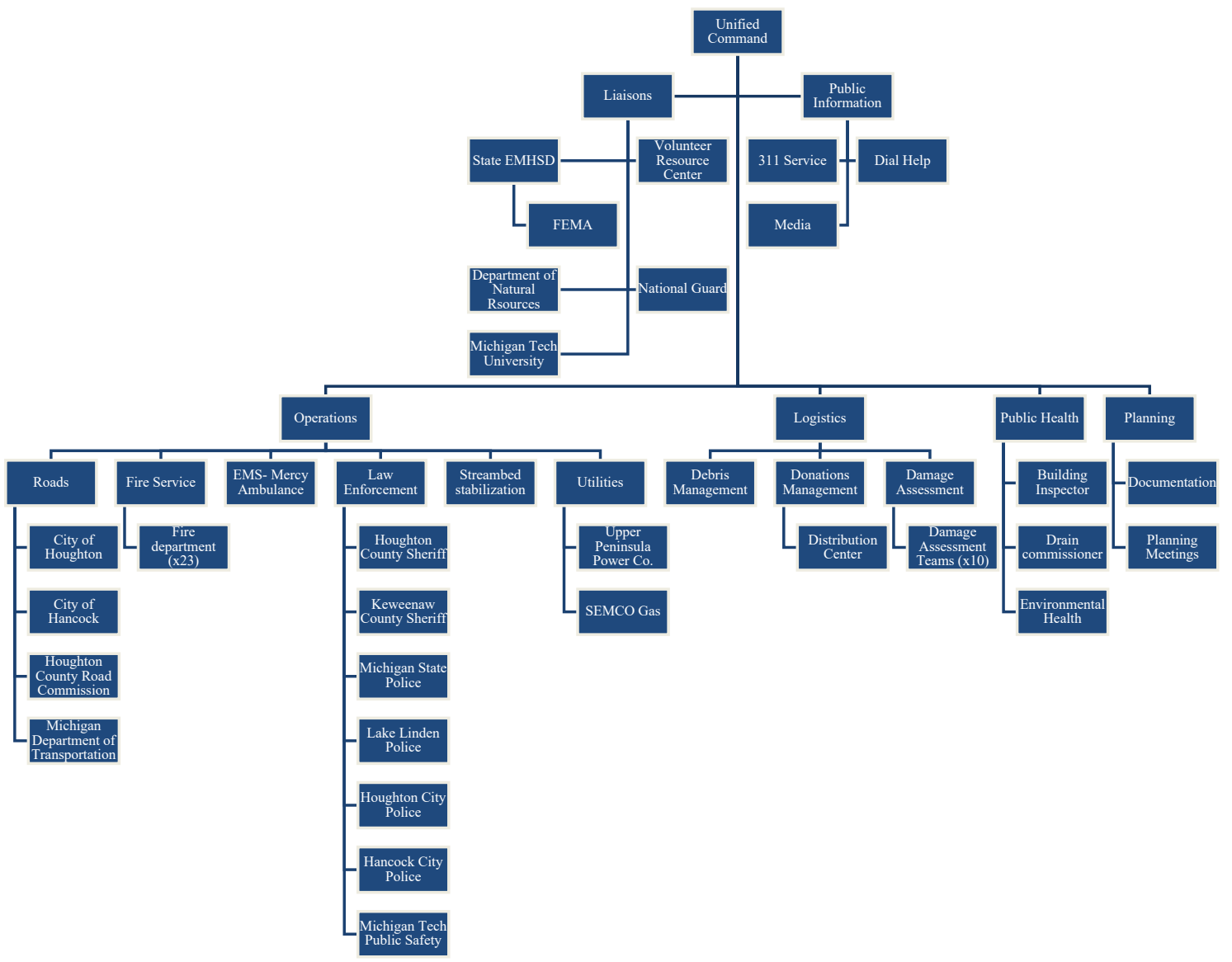

Figure 48: Stabilization Phase Organizational Structure 
The stabilization phase response structure was organized at the county level with each jurisdiction, responding agency, and infrastructure company taking part in twice daily update meetings. These meetings covered:

\section{Morning Briefing}

- Status changes overnight

- Objectives for the day

- Requests for resources

- Questions for other agencies

- Time for one-on-one coordination with other agencies

- Public Information messages to release

\section{Afternoon Planning Meeting}

- Review of accomplishments from the day

- Discussion on plans for overnight

- Discussion of issues of concern that came up during the day

- Changes in resource status

Despite the significant damage to road infrastructure, damage to other infrastructure systems was light. Few power outages were reported in populated areas, some residents lost natural gas service when their road washed out, but again, these outages were very localized. There were no communication outages and cell phone towers were not overwhelmed. The public safety radio system (Michigan Public Safety Communication System (MPSCS)) was heavily loaded in the early hours of the response but not beyond the first 5-8 hours and the system remained usable.

\subsubsection{Interactions}

The interactions in the 2018 flood response are listed below for both the initial response phase and the stabilization phase of the incident.

\subsection{Initial Phase Interactions:}

Unified Command with:

- State of Michigan - Emergency Management and Homeland Security Division of the Michigan State Police (EMHSD)

- Michigan Technological University

- Houghton County Road Commission

- Local hospitals (2)

- Long-term Care facilities (3 involved)

- Mercy EMS (ambulance service)

- Negaunee Regional Communications Center

- Michigan Department of Transportation

- Fire Service (via one liaison) 
- Law Enforcement (via Houghton County Sheriff and Michigan State Police Post Commander)

- Houghton County Building Inspector

- Houghton County Drain Commissioner

- Houghton County Mine Inspector

- Houghton County Board of Commissioners Chairman (Chief Elected Official)

Law Enforcement Liaison (Houghton County Sheriff) with:

- Keweenaw County Sheriff's Office

- Michigan State Police

- Houghton City Police

- Hancock City Police

- Michigan Tech Public Safety and Police Services

Fire Service Liaison (Bootjack Fire Chief) with:

- Stanton Township Fire and First Responders

- Houghton City Fire

- Hancock City Fire

- Ripley Fire

- Quincy-Franklin-Hancock Township Fire

- Hubbell Fire

- Tamarack City Fire

- Chassell Township Fire

- Hurontown Fire and Rescue

- Lake Linden Fire

- Laurium Village Fire

\subsection{Stabilization Phase Interactions:}

Unified Command with:

- State of Michigan - Emergency Management and Homeland Security Division of the Michigan State Police (EMHSD)

- Michigan Technological University

- Michigan Department of Natural Resources

- Volunteer Resource Center

- Operations Section Chief

- Logistics Section Chief

- Public Health Officer

- Negaunee Regional Communications Center

- Local non-profit foundations

- Houghton County Board of Commissioners Chairman (Chief Elected Official) 
EMHSD District Coordinator with:

- State Emergency Operations Center

- FEMA

- Stage Damage Assessment Support Team

Public Information Officer with:

- Local media (radio, print, TV)

- State and Federal Elected Officials

Operations Section Chief with:

- Houghton County Road Commission

- Michigan Department of Transportation

- National Guard

- City of Houghton Public Works

- City of Hancock Public Works

- Mercy EMS (ambulance service)

- Fire Service (via one liaison)

- Law Enforcement (via Houghton County Sheriff and Michigan State Police Post Commander)

- Upper Peninsula Power Company

- SEMCO Energy (natural gas supplier)

- Michigan Department of Energy, Great Lakes and Environment (EGLE) Team Stream assessment team who coordinated with:

- Houghton County Drain Commissioner

- Houghton County Mine Inspector

Logistics Section Chief with:

- Debris management agencies (Waste Management, Houghton County, State of Michigan Department of Corrections)

- Damage Assessment Group leader

- Donations Management Group Leader

Public Health Chief with:

- Western UP Health Department (environmental health)

- Houghton County Building Inspector

Houghton County Board of Commissioners Chairman (Chief Elected Official) with:

- Township, village, city officials

Law Enforcement Liaison (Houghton County Sheriff) with: 
- Keweenaw County Sheriff's Office

- Michigan State Police

- Houghton City Police

- Hancock City Police

- Michigan Tech Public Safety and Police Services

Fire Service Liaison (Bootjack Fire Chief) with:

- Stanton Township Fire and First Responders

- Houghton City Fire

- Hancock City Fire

- Ripley Fire

- Quincy-Franklin-Hancock Township Fire

- Hubbell Fire

- Tamarack City Fire

- Chassell Township Fire

- Hurontown Fire and Rescue

- Lake Linden Fire

- Laurium Village Fire

\subsubsection{Areas of Disequilibrium}

Disequilibrium was present in the 2018 flood in many places. Disequilibrium is the difference between the expected or normal value and the actual value. In this flooding incident, the incident itself was a disequilibrium for the normal or expected. While flash flooding and urban flooding was present in the Houghton County Hazard Mitigation Plan (Western Upper Peninsula Planning and Development Region 2020), significant infrastructure damage due to flooding was not and no recent exercises had been conducted with significant road outages.

Information flow disequilibrium:

- Phone and text reports: During the initial phase of the response, too many agencies were reporting to the Unified Commanders. At the emergency operations center, phone and text messages were overloading the phones. The Emergency Management Coordinator and the Sheriff were on two phones at a time with several calls holding while additional calls were coming in. This continued for the first 4 hours of the incident (approximately 4:30 AM-8:30 AM). The result of this backlog was that agencies that needed decisions to be made were unable to get answers in a timely manner and had to act on their own. It also reduced the situational awareness of the Unified Commanders and they did not have sufficient time to share the information from calls they had received with each other.

- Evacuation: In particular, a decision about evacuating a long-term care facility of residents was needed. A concern about a potential earthen dam breaching and causing a mudslide in the community of Hubbell resulted in a discussion between the Emergency Management Coordinator, the Hubbell Fire Chief, Mercy EMS President, and the longterm care facility manager about evacuating the residents of the facility. The overload of 
information and the lack of ability to make calls out of the Emergency Operation Center (due to the significant number in incoming calls) could have significantly impacted this critical operation.

- Damage reports: Receiving damage reports (road outages in particular) was critical to the response. Road outages not only impede the general public but impact the ability to get emergency vehicles to areas in need. During the initial stages of the flood, several communities were completely cutoff from outside support due to road outages. Reports of outages were made to the Emergency Operations Center and a list was compiled. But with no GIS support available and Houghton County's reliance on a free version Google Suite with limited map sharing options, setting up a common shared map that could be easily relayed to agencies and the public took more than 8 hours. During the remainder of the initial and stabilization phases of the incident, only a list of road closures was actively maintained with the locations looked up on an online map as needed.

- Local Amateur Radio Emergency Service (ARES): According to the Emergency Operation Plan, during an emergency, local amateur radio operators can be called to assist with information gathering. Local Amateur Radio Emergency Service (ARES) members were asked to setup a radio communications network (net) to take damage reports from their members scattered around the county. This net was maintained for the first 14 hours of the incident but only received 4 reports.

- Damage assessment: FEMA requires a preliminary damage assessment within 72 hours of the incident stabilizing. For this task, fire fighters, search and rescue members and emergency management coordinators from other counties volunteered to go door to door across the affected areas of the county. By this time, staff from the Emergency Management and Homeland Security Division of the Michigan State Police (EMHSD) had arrived with a damage assessment system using GIS and iPads. Each team was quickly trained and send out on assignments to cover the affected areas taking pictures, rating damage (FEMA uses a 1-5 scale) and recording the results. Unfortunately, the first day of damage assessment was poorly organized with some areas being missed, other areas being covered twice, and additional areas not consistently assessed. Starting the second day, a damage assessment group coordinator was chosen (who was also not one of the Unified Commanders) and designed the team assignments, reiterated the objective and methods to use, and was able to correct the errors of the first day.

Logistics

- Emergency Operations Center (EOC) and County Offices: The Houghton County Emergency Operation Center was located approximately 15 miles north of the county offices. Due to road outages, it was decided to setup an alternative EOC at the Sheriff's Office. According to the county plan, the alternate EOC was located in the basement of the courthouse, however, that space was flooded with 8 inches of water during the early parts of the incident. Incidentally, that space also included the office (including the computer, hard copy Emergency Operation Plans, etc. of the Emergency Management Coordinator). The county plans did not account for both the primary and alternate EOC's to be inaccessible in a wide-scale emergency. The result was an ad hoc EOC with the equipment the Sheriff's Office and other responders had from home. 
- Donations: there was an outpouring of donations from around the country as news of the flood spread including semi-truck loads of paper products from Kimberly Clark, power tools from Milwaukee Tool, trash pumps from the Eagle Mine in Marquette, barbecue meals from Arkansas, truckloads of bottled water, and many other items. The local emergency plan was not setup to accommodate the large volume of donations and there was not a significant number of community members requesting items. This posed a significant challenge for accepting, inventorying, and then distributing these donations. At the end of the incident, donations management was the most time-consuming component of the response for the Incident Command Team.

\subsubsection{Measurement of Complexity}

The measurement of complexity is, as mentioned previously in this dissertation, based on perspective. For this analysis, the perspective is from the Unified Command as they were organizing the response (Table 4). The circular representation (Figure 49) shows the interactions of the Unified Command, agencies, and responders during the initial phase. In the initial phase, the overlap in the inner (agency) circles indicates disequilibrium- where the Unified Command could not accommodate the agencies fast enough to eliminate delay. In contrast, Figure 50 shows that there are only two points of interaction (the scheduled meetings) and all agencies overlay each other on the inner circle.

Unified Command

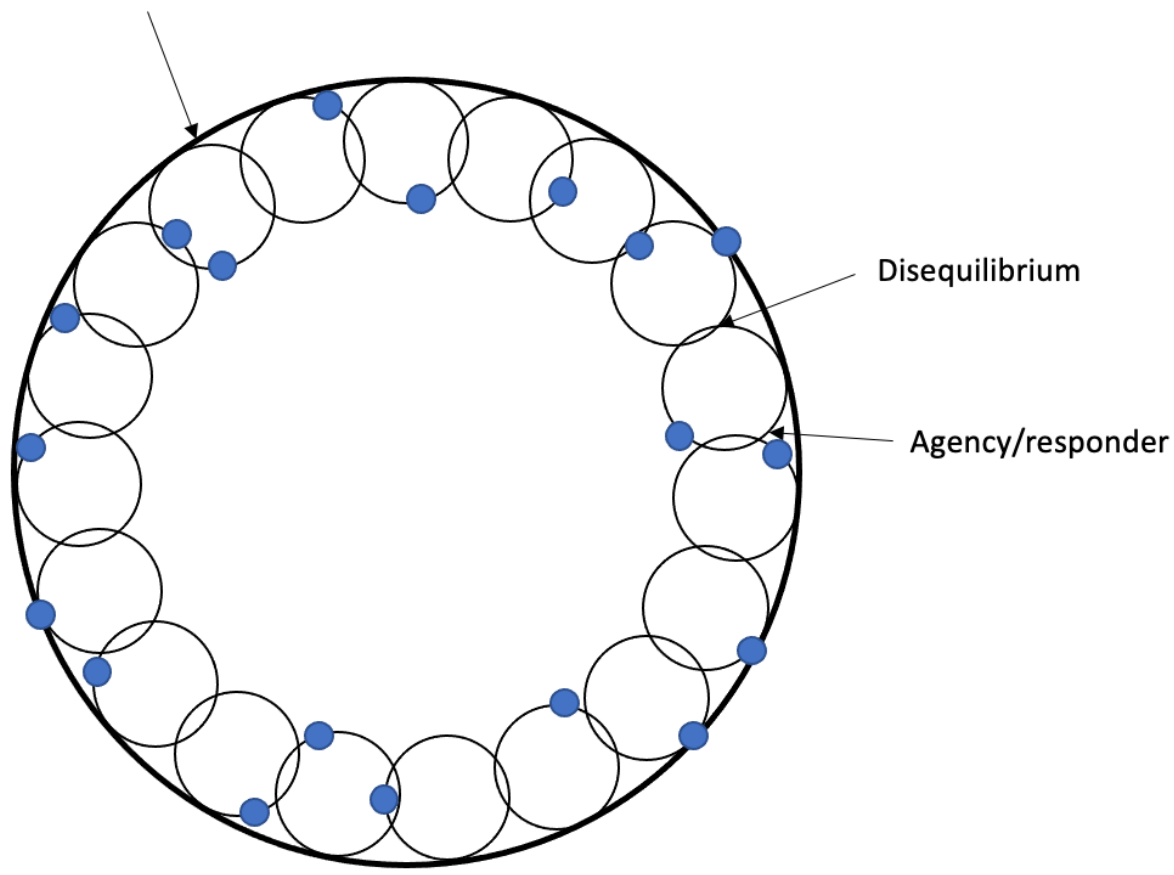

Figure 49: Unified Command-Agency Interactions during the initial phase of the 2018 flood. Note the overlap of the inner circles indicates disequilibrium. 


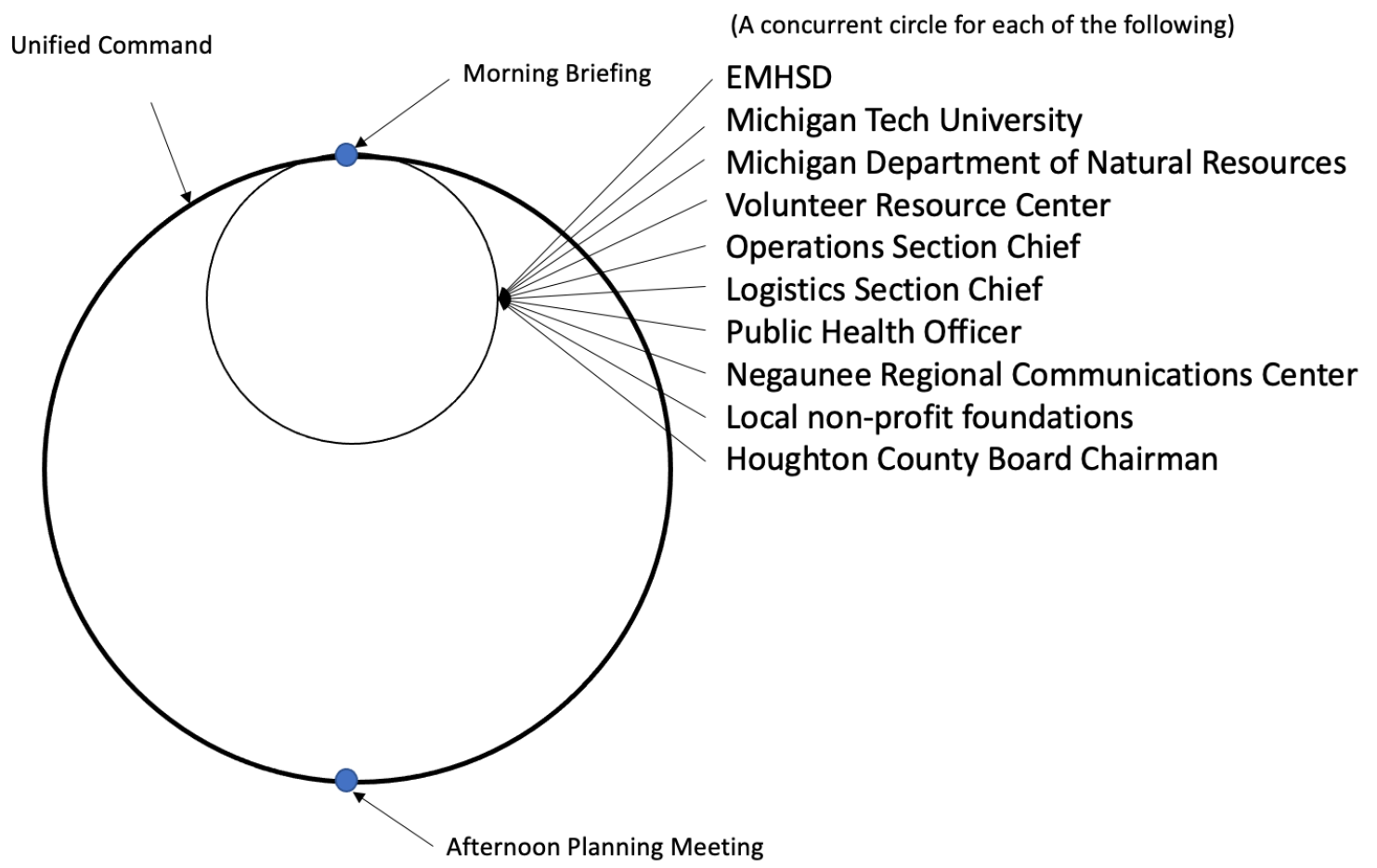

Figure 50: Unified Command-Agency Interactions during the stabilization phase of the 2018 flood response.

Table 4: Complexity by operational period

\begin{tabular}{llll}
\hline Operational Period & $\begin{array}{l}\text { Total ICS } \\
\text { Interactions }\end{array}$ & $\begin{array}{l}\text { Interactions with } \\
\text { Unified Command }\end{array}$ & Complexity Measure \\
\hline 1 (initial) & 42 & 19 & 19 \\
2 (Stabilization) & 60 & 10 & 10
\end{tabular}

\subsubsection{Driving Interactions}

There was no single driving interaction in this incident, but rather there was a set of interactions that when removed, significantly reduced the disequilibrium and increased the efficiency of the response. Reducing the number of direct reports to the Unified Command and assigning Section Chiefs (specifically Operation, Logistics, and Public Health) removed the backlog of messages and allowed the Unified Command to move from a reactionary operation to a planning and proactive operational stance. Table 4 shows the complexity of the initial and stabilization phases of the incident. 


\subsubsection{Summary}

Recognizing the need to reduce the information flow failures during the initial phase of the incident, the Unified Command began creating Section Chiefs to reduce the number of interactions the Unified Command needed to have. While the total number of interactions in the system actually increased, the interactions with the Unified Command decreased, reducing the complexity. This is shown by the smaller disequilibrium by reducing the delay in the information flow. The second thing the Unified Command did was to request updates only twice a day. By requesting updates only at these times, that effectively consolidated the interactions together into two larger (longer) interactions.

By examining the number and nature of the interactions, the complexity was examined and reduced for the Unified Command. Like the previous case, this incident demonstrates that measuring complexity by the number of interactions from a given symmetry (perspective). This is also a decrease in disequilibrium for the system. The idea of having fewer direct reports is also reinforced by FEMA who recommends that a single supervisor oversees no more than 5-7 people (Federal Emergency Management Agency 2018c) and is supported by the research of Miller (1956), mentioned earlier in this dissertation. 


\section{Conclusions and Recommendations}

\subsection{Conclusions}

The objective of this research was to determine a measure of complexity and system characteristics that indicate likely points of information flow failure in project management. Using methods to visualize the number and nature of component interactions, the disequilibrium can be detected, and the critical interactions identified. Identifying these critical interactions allows the project manager to reduce the number of interactions for the desired symmetry, decreasing the complexity, and the potential for disequilibrium leading to a reduction in information flow failures. As demonstrated in the case studies, not all interactions can be reduced without information loss. Further, reducing the complexity for a particular perspective or symmetry, may increase complexity for other areas of the system.

The information flow failures that negatively impact the efficiency of a project or response are points of disequilibrium that occur at interactions between two components of a system. As the number of component intersections increase, the degrees of freedom increase, which increases the number of points that can be in disequilibrium. Therefore, by reducing the number of interactions (reducing the complexity), the opportunities for disequilibrium are reduced.

This research also has discussed project scaling and the application of the measure of complexity. As project systems scale, the measure of complexity continues to apply. Larger systems, as shown in the case studies, have a greater number of components and interactions. But this alone does not indicate higher complexity. The complexity is based on the symmetry; therefore, overall system interactions may increase, but the interactions given a chosen symmetry may be lowerleading to a lower measure of complexity. It is interesting to note that this conclusion of the research (limiting the number of interactions to reduce complexity, and therefore information flow failures) mirrors the guidance from FEMA regarding span of control, or the maximum number of people or groups a supervisor can effectively manage. FEMA's guidance states supervising more than 5 people can lead to communication failures. This dissertation does not specify a maximum number of interactions before failure, but this is an area for future work.

Also of note are the connections between this research and the Kalman minimal realization of these systems. Since signals may not be directly observable (recall that projects do not necessarily give off signals), the ability to determine if an interaction is in disequilibrium indirectly would be helpful. For example, in an emergency that is taking place over a large geographic area, the cycle times for search teams in a remote area may not be measurable from the command post. But, based on the remote staging area's manager, and other search teams operating at that location, the state of a particular team could be estimated.

\subsection{Limitations}

The research in this dissertation has made some assumptions and has limitations in its application. However, these limitations do not change the overarching measure of complexity as a function of 
the number and nature of interactions for a system. Moreover, the methods used to analyze and visualize the disequilibrium in a system may not all apply to all types of systems as effectively.

First, this research assumes that there is data available such as cycle times of components, the organization, or relationship between components of a project or emergency response. It also assumes that there is time for processing that data, even at a basic level during the project or incident.

Second, the images in this dissertation, use the assumption that cycle times are constant for all of a particular type of component (search team for example). This is not necessarily the case as these cycle times may vary. However, that does not change the underlying concept that the circle circumference represents the cycle time of the component.

Third, it is important to recognize that the analysis in the case studies represents snapshots in time of those projects. As the projects scale up and down, the system changes and the measure of complexity can change as well.

\subsection{Contributions}

This research contributes to the body of knowledge by providing quantitative methods for measuring system complexity. The measurement can be used in planning and analyzing projects or incidents. Practitioners in project and emergency management can use the knowledge of the number and nature of interactions in their systems to reduce the potential for information flow failures. Project and emergency managers can build into their plans, a time for reflection on project information flow failures and examine what driving interactions are causing that disequilibrium. Not all aspects of each project can be measured or planned, but knowledge of the concept that fewer interactions reduces the potential for disequilibrium in a system, which reduces the opportunities of information flow failures, can help managers improve their projects. With information flow failures being nearly universally cited in emergency response and exercise After-Action Reports as a cause of inefficiency, this research can help bridge that shortcoming.

\subsection{Recommendations for future work}

There are several areas of related research that were beyond the scope of this project but would further the goal of improving efficiency in project management:

- A discussion of the behavior of agents (components) that is unplanned and emergent behavior would be beneficial. This research assumed that the components or resources followed instructions and assignments. However, in real projects, resources may (will) improvise or simply ignore instructions and assignments if they choose. One question to address is: If the disequilibrium reaches a critical point, and the agent decides not to wait, what will that agent do and how does that impact the complexity of the system when agents create new interactions? This direction would likely be a fruitful collaboration with research in behavioral science.

- The impact of exercises and training on disequilibrium should be explored. FEMA requires responders and emergency management coordinators to meet annual exercise and 
training requirements for grant program compliance (the Emergency Management Performance Grant for example). Data on how effective this requirement is in reducing disequilibrium would be valuable and could inform this often expensive and very timeconsuming requirement in the future.

- Additional research into the geographic realm of complexity and disequilibrium should be made. Already the author is conducting research into the differences in lost person behavior using different methods such as least cost path in comparison to a Bayesian method. This research would look at the differences in disequilibrium while potentially holding the number of interactions constant (i.e., same resources available, different search methods).

- While discussed briefly in this dissertation, examining disequilibrium through the lens of network stability, resilience, and robustness of complex infrastructure networks, is an area of research that could be explored further. The relationship between interdependent infrastructure components and the component interactions could be similar to the work conducted in this dissertation. Fundamentally, this would try to answer the question similar to one asked above: How much disequilibrium can a system handle before it breaks?

These additional areas of future research would continue this work and further develop use cases for the metric of complexity developed in this dissertation. 


\section{References}

Anderson, A. I., Compton, D., and Mason, T. (2004). "Managing in a Dangerous World-The National Incident Management System." Engineering Management Journal, Taylor \& Francis, 16(4), 3-9.

Austin, S., Newton, A., Steele, J., and Waskett, P. (2002). "Modelling and managing project complexity." International Journal of project management, Elsevier, 20(3), 191-198.

Axelrod, R. M., Axelrod, R., and Cohen, M. D. (1999). Harnessing Complexity: Organizational Implications of a Scientific Frontier. Free Press.

Bennet, B. (2011). "Effective emergency management: A closer look the incident command system." Professional Safety, American Society of Safety Engineers, 56(11), 28-37.

Bertelsen, S. (2003). "Complexity--Construction in a new Perspective." IGLC-11, Blacksburg, Virginia.

Bertelsen, S., and Koskela, L. (2005). "Approaches to managing complexity in project production." Proc. of 13th IGLC, Sydney, Australia, 65-71.

Bigley, G. A., and Roberts, K. H. (2001). "The incident command system: High-reliability organizing for complex and volatile task environments." Academy of Management Journal, 44(6), 1281-1299.

Brook, P. (2016). "On the Nature of Systems of Systems." 26th Annual INCOSE International Symposium, 1477-1493.

Comfort, L. K., Ko, K., and Zagorecki, A. (2004). "Coordination in rapidly evolving disaster response systems: The role of information." American Behavioral Scientist, 48(3), 295-313.

Comfort, L. K., Oh, N., and Ertan, G. (2009). "The dynamics of disaster recovery: Resilience and entropy in hurricane response systems 2005-2008." Public Organization Review, 9(4), 309323.

Comfort, L. K., Siciliano, M. D., and Okada, A. (2011). "Resilience, Entropy, and Efficiency in Crisis Management: The January 12, 2010, Haiti Earthquake." Risk, Hazards \& Crisis in Public Policy, Blackwell Publishing Ltd, 2(3), 1-25.

Crutchfield, J. P., and Young, K. (1989). "Inferring statistical complexity." Physical Review Letters, APS, 63(2), 105.

Dao, B., Kermanshachi, S., Shane, J., and Anderson, S. (2016). "Project Complexity Assessment and Management Tool." Procedia Engineering, Elsevier, 145, 491-496.

Eusgeld, I., Nan, C., and Dietz, S. (2011). "'System-of-systems' approach for interdependent critical infrastructures." Reliability Engineering \& System Safety, Elsevier, 96(6), 679-686. 
Favero, G. T. (1999). "Flexibility of the incident command system to respond to domestic terrorism." Monterey, California: Naval Postgraduate School.

Federal Emergency Management Agency. (2018a). 2017 Hurricane Season FEMA After-Action Report.

Federal Emergency Management Agency. (2018b). Preliminary Damage Assessment Report, Michigan 4381-DR-MI.

Federal Emergency Management Agency. (2018c). ICS 100, Introduction to the Incident Command System, Student Manual. FEMA, Emergency Management Institute.

Federal Emergency Management Agency. (2020). Homeland Security Exercise and Evaluation Program (HSEEP) Course.

FEMA. (2018). "ICS 100, Introduction to the Incident Command System." FEMA, Emergency Management Institute, (November), 160.

Flood, R. L. (1987). "Complexity: A definition by construction of a conceptual framework." Systems Research, 4(3), 177-185.

Gell-Mann, M. (2002). "What is complexity?" Complexity and Industrial Clusters, Springer, 13-24.

Gell-Mann, M., and Lloyd, S. (2004). "Effective complexity." Nonextensive entropy, 387-398.

Gidado, K. I. (1996). "Project complexity: The focal point of construction production planning." Construction Management \& Economics, Taylor \& Francis, 14(3), 213-225.

Gideon, J. M., Dagli, C. H., and Miller, A. (2005). "Taxonomy of Systems-of-Systems." Conference on Sysems Engineering Resarch, Institute of Electrical and Electronics Enginer (IEEE).

Gransberg, D. D., Shane, J. S., Strong, K., and del Puerto, C. L. (2013). "Project Complexity Mapping in Five Dimensions for Complex Transportation Projects." Journal of Management in Engineering, 29(4), 316-326.

Gribble, S. D. (2001). "Robustness in complex systems." Proceedings eighth workshop on hot topics in operating systems, 21-26.

Grinter, R. E., Herbsleb, J. D., and Perry, D. E. (1999). “Geography of coordination: Dealing with distance in R\&D work." Proceedings of the International ACM SIGGROUP Conference on Supporting Group Work, 306-315.

Hall, J. L. (2003). "Columbia and Challenger: Organizational failure at NASA." Space Policy, 19(4), 239-247.

Holden, R., Val, D. V., Burkhard, R., and Nodwell, S. (2013). "A network flow model for interdependent infrastructures at the local scale." Safety Science, Elsevier Ltd, 53, 51-60. 
ICCPM. (2012). Complex Project Manager Competency Standards. International Centre for Complex Project Management (ICCPM), Commonwelth of Australia (Department of Defence).

Iyer, S., Killingback, T., Sundaram, B., and Wang, Z. (2013). "Attack Robustness and Centrality of Complex Networks." PLOS ONE, 8(4).

Jensen, J., and Jr., Waugh, W. L. (2014). "The United States' Experience with the Incident Command System: What We Think We Know and What We Need to Know More About." Journal of Contingencies and Crisis Management, 22(1), 5-17.

Johansson, J., and Hassel, H. (2010). "An approach for modelling interdependent infrastructures in the context of vulnerability analysis." Reliability Engineering \& System Safety, Elsevier, 95(12), 1335-1344.

Jordan, E., and Javernick-Will, A. (2013). "Indicators of Community Recovery: Content Analysis and Delphi Approach." Natural Hazards Review, 14(1), 21-28.

Jordan, E., Javernick-Will, A., and Tierney, K. (2016). "Post-tsunami recovery in Tamil Nadu, India: combined social and infrastructural outcomes." Natural Hazards, Springer, 84(2), 13271347.

Leonidas, T. (2011). Information flow. The Logic of Distributed systems. Health facilities management.

Little, R. G. (2003). "Toward more robust infrastructure: observations on improving the resilience and reliability of critical systems." System Sciences, 2003. Proceedings of the 36th Annual Hawaii International Conference on System Sciences.

López-Ruiz, R., Mancini, H., and Calbet, X. (1995). "A Statistical Measure of Complexity." Physics Letters A, 209(5-6), 321-326.

Manoj, B. S., Hubenko Baker, A., Baker, A. H., and Hubenko Baker, A. (2007). "Communication Challenges in Emergency Response." Communications of the ACM, 50(3), 56.

Manson, S. M. (2001). "Simplifying complexity: a review of complexity theory." Geoforum, Elsevier, 32(3), 405-414.

Matisziw, T. C., Murray, A. T., and Grubesic, T. H. (2008). "Exploring the vulnerability of network infrastructure to disruption." The Annals of Regional Science, 43(2), 307-321.

Matisziw, T. C. T., Grubesic, T. H. T., and Guo, J. (2012). "Robustness Elasticity in Complex Networks." PLOS ONE, Public Library of Science, 7(7), e39788.

Matisziw, T., Murray, A., and Grubesic, T. (2010). "Strategic Network Restoration." Networks and Spatial Economics, 345-361. 
McCabe, T. J. (1976). "A Complexity Measure." IEEE Transactions on Software Engineering, SE2(4), 308-320.

Miller, G. A. (1956). "The magical number seven, plus or minus two: some limits on our capacity for processing information." Psychological Review, American Psychological Association, US, 63(2), 81-97.

Monge, P. R., Contractor, N. S., Peter, R., Contractor, P. S., Noshir, S., and others. (2003). Theories of communication networks. Oxford University Press, USA.

Moynihan, D. P. (2009). "The network governance of crisis response: Case studies of incident command systems." Journal of Public Administration Research and Theory, 19(4), 895-915.

Munns, A. K., and Bjeirmi, B. F. (1996). "The role of project management in achieving project success." International Journal of Project Management, 14(2), 81-87.

Murray, A., Matisziw, T., and Grubesic, T. (2007). "Critical network infrastructure analysis: interdiction and system flow." Journal of Geographical Systems, 103-117.

Nicholson, W. C., others, and Nicholson, JD, W. C. (2006). "The Role of the Incident Command System." Journal of Emergency Management, 4(1), 19-22.

Oomes, A. H. J., and Neef, R. M. (2005). "Scaling-up support for emergency response organizations." Proceedings of ISCRAM 2005, (April), 29-41.

Owens, J., Ahn, J., Shane, J. S., Strong, K. C., and Gransberg, D. D. (2012). "Defining Complex Project Management of Large U.S. Transportation Projects: A Comparative Case Study Analysis." Public Works Management and Policy, Sage Publications Sage CA: Los Angeles, CA, 17(2), 170-188.

Paina, L., and Peters, D. H. (2012). "Understanding pathways for scaling up health services through the lens of complex adaptive systems." Health Policy and Planning, 27(5), 365-373.

Paul, G., Tanizawa, T., Havlin, S., and Stanley, H. E. (2004). "Optimization of robustness of complex networks." The European Physical Journal B, Springer, 38(2), 187-191.

Payne, J. W. (1976). "Task complexity and contingent processing in decision making: An information search and protocol analysis." Organizational Behavior and Human Performance, 16(2), 366-387.

Phelan, S. E. (1999). "A note on the correspondence between complexity and systems theory." Systemic Practice and Action Research, Springer, 12(3), 237-246.

Prange, S. J. (2013). "Sustainable Design Practices Result in" More Than Just a Bridge." Green Streets, Highways, and Development 2013: Advancing the Practice, 21-30.

Provan, K. G., and Kenis, P. (2008). “Modes of network governance: Structure, management, and 
effectiveness." Journal of Public Administration Research and Theory, 18(2), 229-252.

Remington, K., and Pollack, J. (2016). Tools for Complex Projects. Tools for Complex Projects, Gower.

Richardson, B. K., and Byers, L. (2006). "Communication studies and emergency management: Common ground, contributions, and future research opportunities for two emerging disciplines." Disciplines, disasters and emergency management: The convergence and divergence of concepts, issues and trends from the research literature.

Rinaldi, S. M. (2004). "Modeling and simulating critical infrastructures and their interdependencies." 37th Annual Hawaii International Conference on System Sciences, 2004. Proceedings of the, 8--pp.

Safapour, E., Kermanshachi, S., Habibi, M., and Shane, J. (2018). "Resource-based exploratory analysis of project complexity impact on phase-based cost performance behavior." Proceedings of Construction Research Congress, 2-4.

Simpson, N. C., and Hancock, P. G. (2009). "Fifty years of operational research and emergency response." Journal of the Operational Research Society, Springer, 60(1), S126--S139.

Texas A\&M Engineering Extension Service (TEEX). (2016). Emergency Operations Center (EOC) Operations and Planning for All-Hazards Events. Federal Emergency Magement Agency, Collage Station.

Vidal, L.-A., Marle, F., and Bocquet, J.-C. (2011). "Measuring project complexity using the Analytic Hierarchy Process." International Journal of Project Management, Elsevier, 29(6), 718-727.

Weick, K. E., Sutcliffe, K. M., and Obstfeld, D. (2008). Organizing for High Reliability: Process of Collective Mindfulness. Crisis Management.

Western Upper Peninsula Planning and Development Region. (2020). Houghton County Hazard Mitigation Plan. Houghton.

Whitty, S. J., and Maylor, H. (2007). "And then came complex project management." 21st IPMA World Congress on Project Management, 371-376.

Yang, I.-T., and loannou, P. G. (2001). "Resource-driven scheduling for repetitive projects: A pullsystem approach." Proceedings of the 9th International Group for Lean Construction Conference, Singapore, 365-377.

Yu, L., and Lai, K. K. (2011). "A distance-based group decision-making methodology for multiperson multi-criteria emergency decision support." Decision Support Systems, Elsevier, 51(2), 307-315.

Zachary, W. W. (1977). "An information flow model for conflict and fission in small groups." Journal of anthropological research, University of New Mexico, 33(4), 452-473. 


\section{A Appendix: Additional Plots of Varying Frequency and Delay}

The plots in this appendix continue the experiments described in Chapter 3 . The first two plots (Figure 51 and Figure 52) show varying frequencies. The third and fourth plots (Figure 53 and Figure 54) show the results of varying the delay on the second component (the excavator). The frequency of the truck cycle remains constant (at 1CPT) and the frequency of the excavator also remains constant at 12CPT. However, the delay (wait time) for the excavator is varied from time= 0 units to time $=0.1$ units. The third set of plots (Figure 55 and Figure 56) show the results of varying both frequency and delay. 

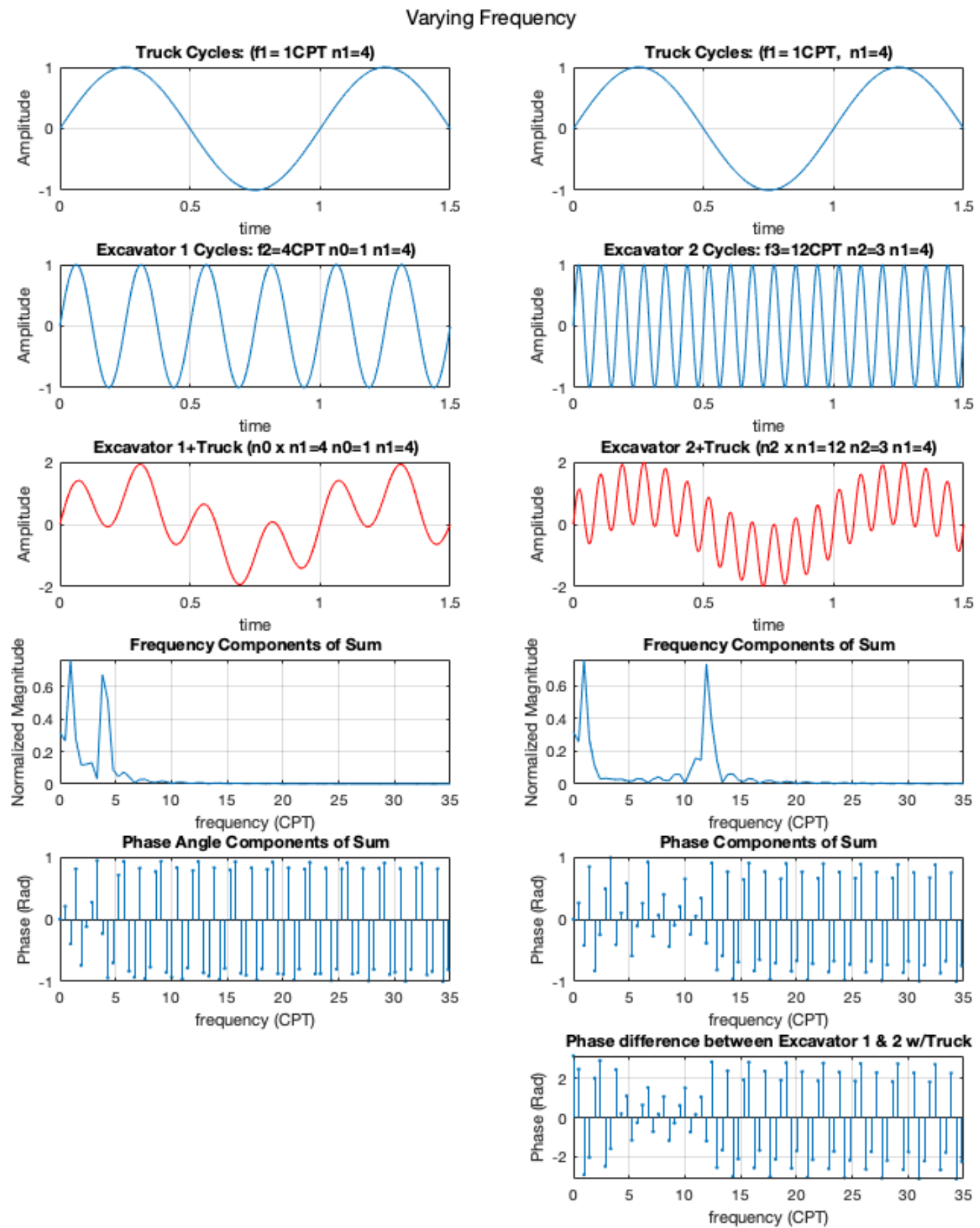

Figure 51: Varying the frequency and comparing the summation waveform, FFT magnitude, and phase calculations shows that each FFT magnitude plot has the frequency components clearly visible as spikes on the normalized magnitude plot. The phase angle of each component is plotted as well as comparison to the base case (left side). 

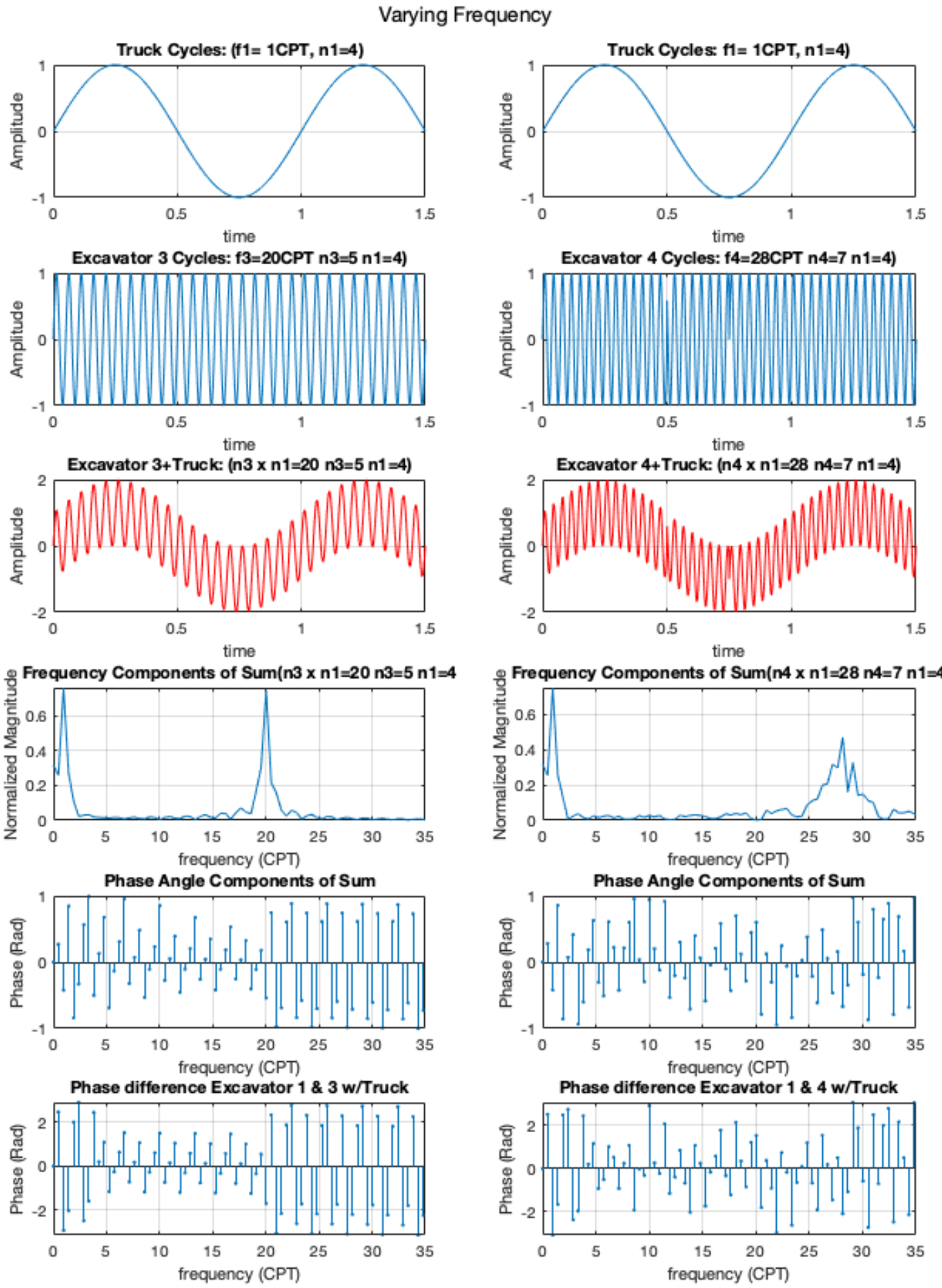

Figure 52: Continuation of figure 48: Varying the frequency and comparing the summation waveform, FFT magnitude, and phase calculations shows that each FFT magnitude plot has the frequency components clearly visible as spikes on the normalized magnitude plot. 


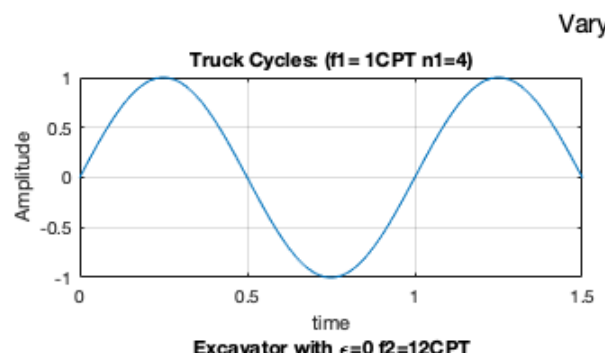

Varying Delay
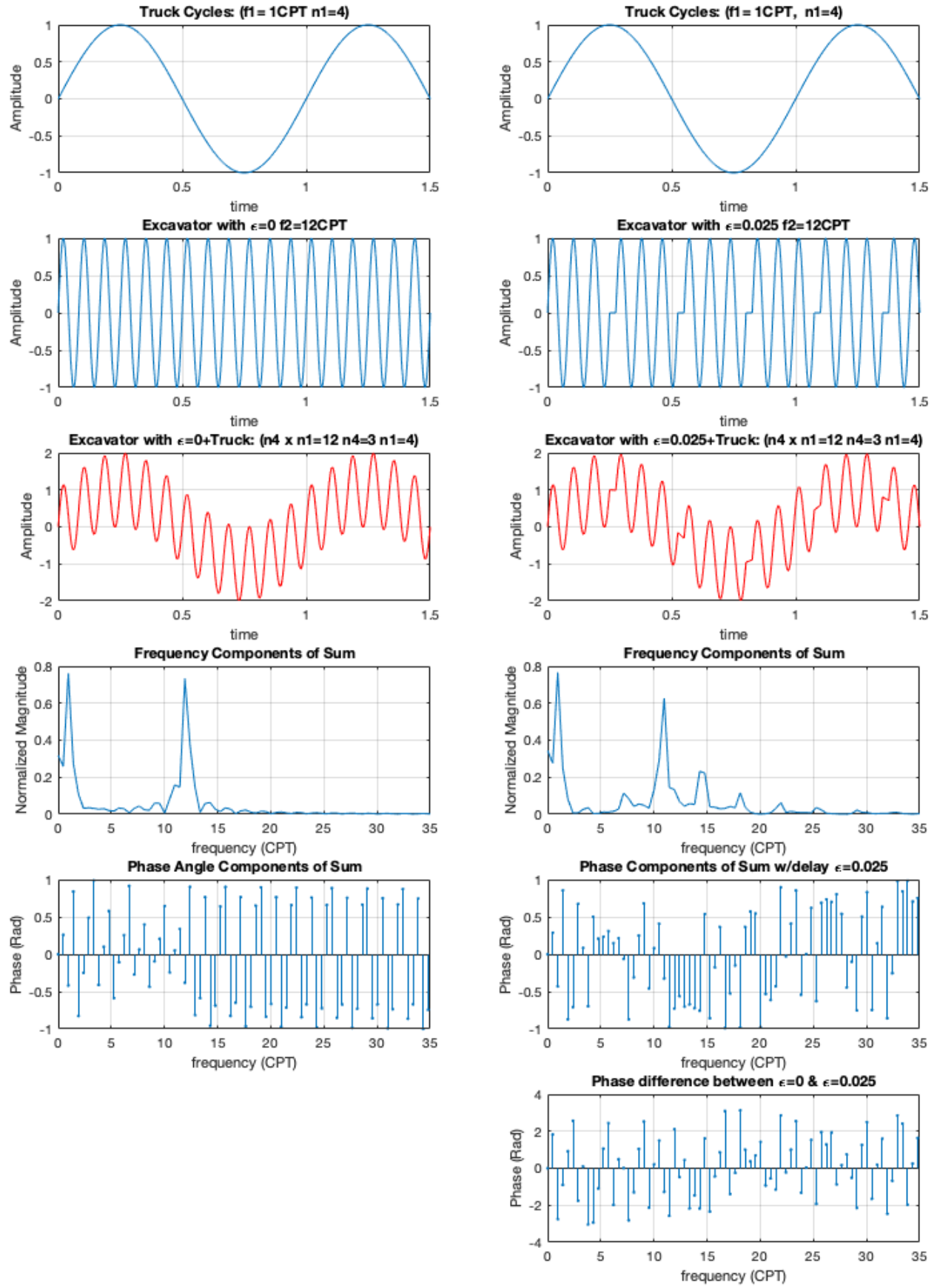

Figure 53: Plots of varying delay while holding the frequencies constant. The plots on the left are with no delay (balanced system). The plots on the right are with a delay of $\varepsilon=0.025$. The phase angle difference plot on the bottom is the difference in phase angle from the control (plot with $\varepsilon=0$ ) and this iteration (plot with $\varepsilon=0.025$ ). 


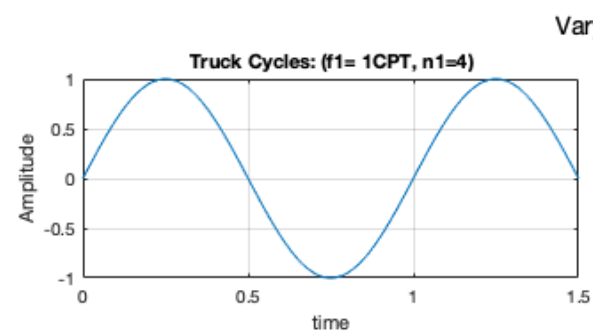

Varying Delay
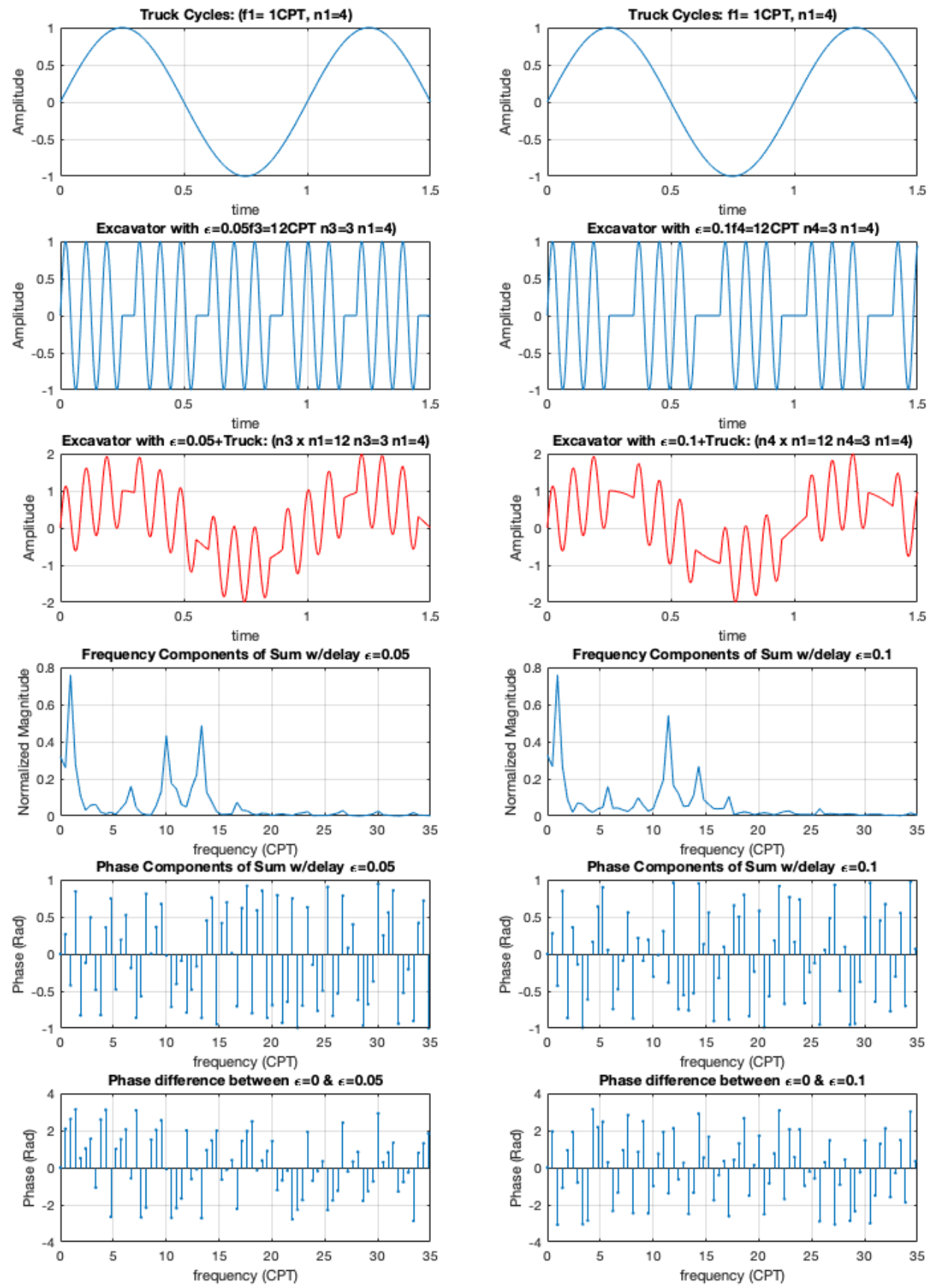

Figure 54: Continuation from figure A-3. Continuing to increase the delay while holding the frequencies constant. The plots on the left are with a delay of $\varepsilon=0.05$. The plots on the right are with a delay of $\varepsilon=0.1$. The FFT in plot $(3,2)$ shows that the delay in the excavator plot is growing large enough that it effectively acts as another frequency in the system. 

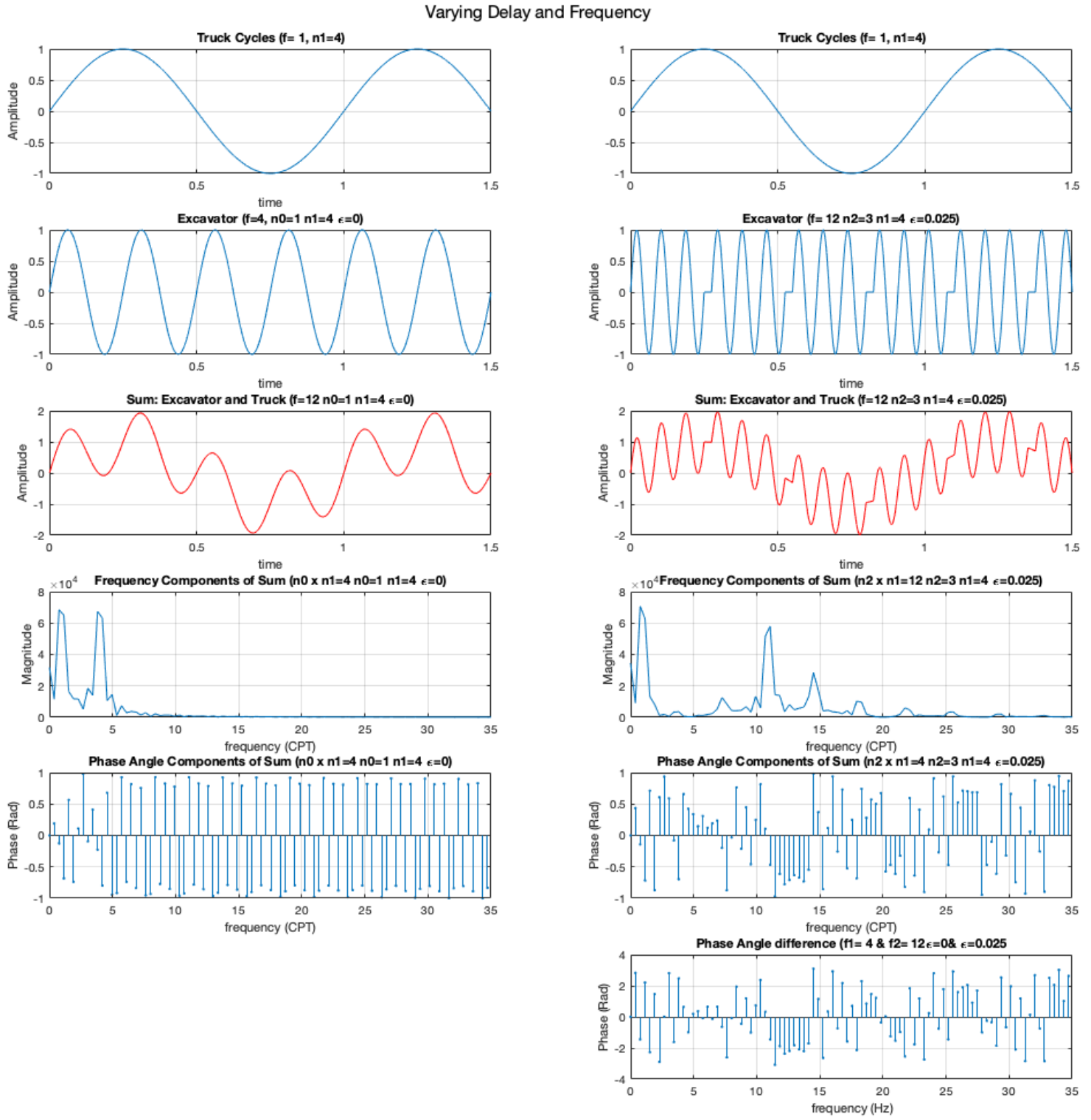

Figure 55: Varying both the frequency and delay and comparing the result to the baseline with two frequencies and no delay. 

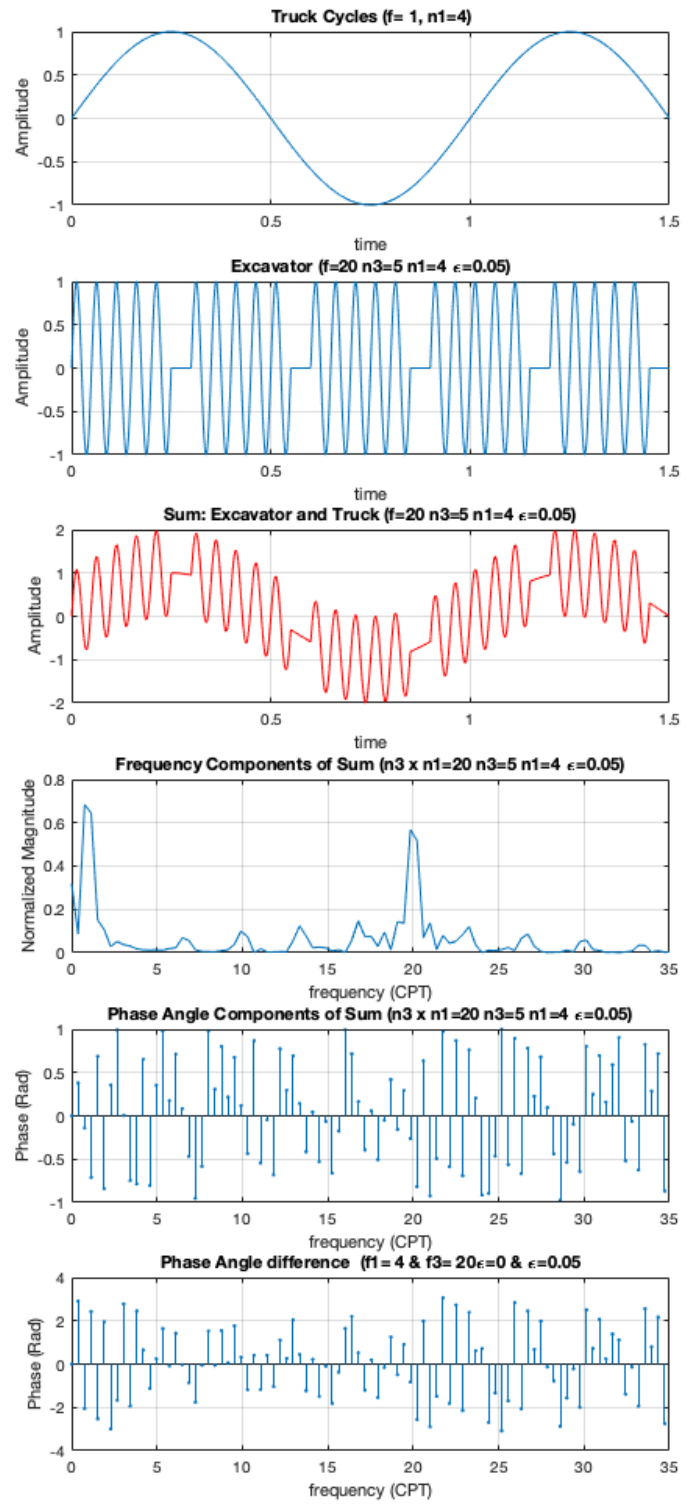
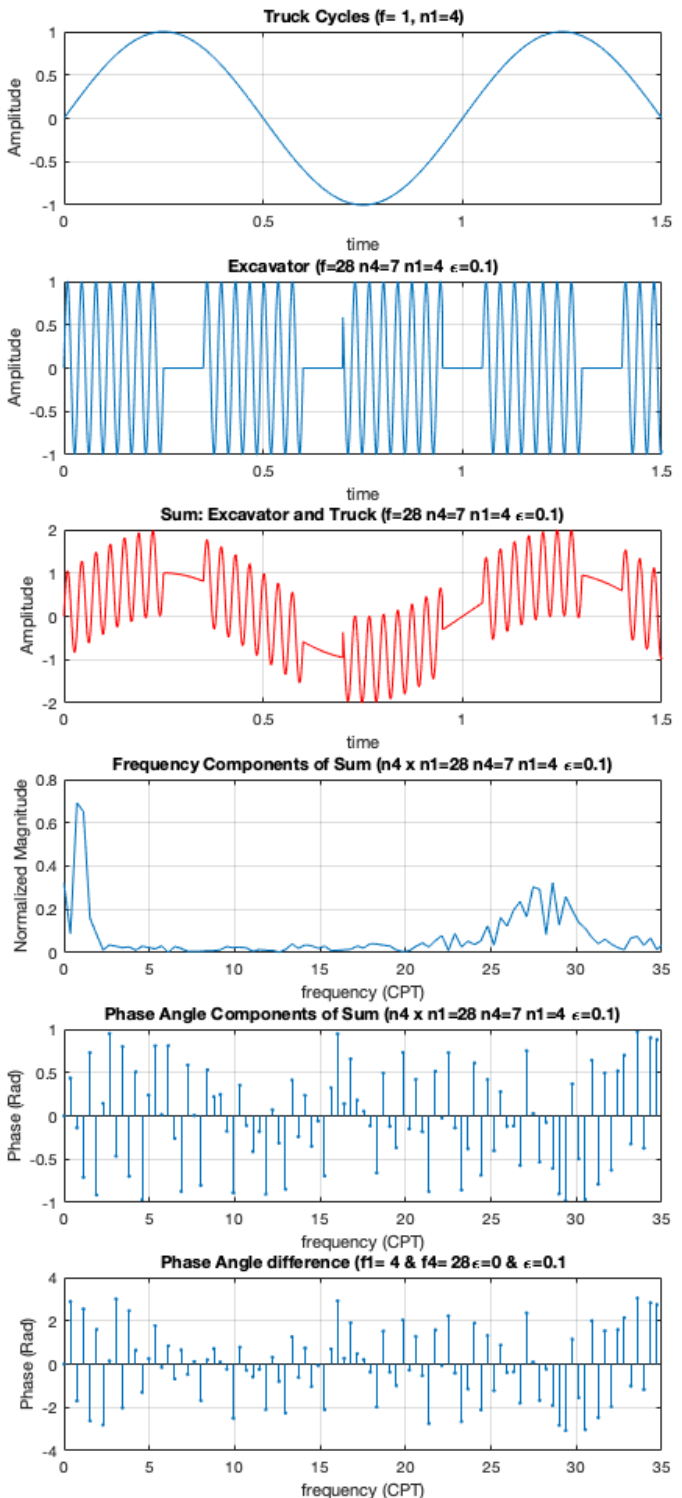

Figure 56: Continuation of figure 54. Varying both the frequency and delay and comparing the result to the baseline with two frequencies and no delay. 


\section{B Copyright Documentation}

Figure 43: "6-Hour Precipitation". Source: National Oceanographic and Atmospheric Administration. Provided by: National Weather Service, July 2018. 